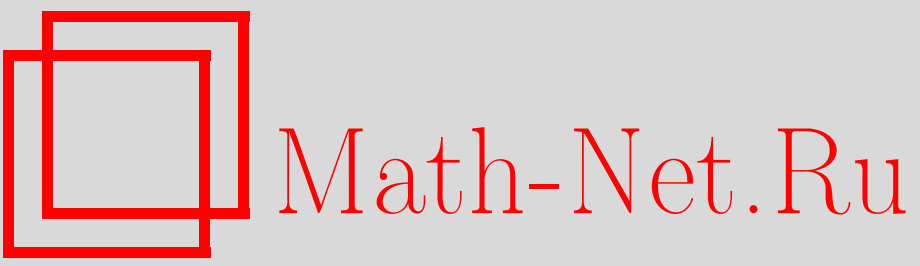

В. И. Данилов, Г. А. Кошевой, Массивы и комбинаторика таблиц Юнга, УМН, 2005, том 60, выпуск 2, 79-142

DOI: https://doi.org/10.4213/rm1402

Использование Общероссийского математического портала Math-Net.Ru подразумевает, что вы прочитали и согласны с пользовательским соглашением

http://www.mathnet.ru/rus/agreement

Параметры загрузки:

IP: 54.174 .149 .18

26 апреля 2023 г., 15:41:35 


\title{
МАССИВЫ И КОМБИНАТОРИКА ТАБЛИЦ ЮНГА
}

\author{
В. И. ДАНИЛов, Г. А. КОшЕВОЙ
}

\begin{abstract}
Классическая теория таблиц Юнга излагается на сравнительно новом и нетрадиционном язьке массивов. Отталкиваясь от привычных операций (или алгоритмов) вставки и сдвига (jeu de taquin), мы вводим более элементарные операции над массивами. Снабженные этими операциями, массивы образуют объект, который можно назвать бикристаллом. Изложение этого формализма, опирающегося на теорему о коммутировании вертикальных и горизонтальных операций, занимает первую часть. Во второй части аппарат массивов применяется для изложения таких разделов теории таблиц Юнга, как плактический моноид, правило Литтлвуда-Ричардсона, соответствие Робинсона-Шенстеда-Кнута, дуальные таблицы, плоские разбиения и т. п.

Библиография: 53 названия.
\end{abstract}

\section{СОДЕРЖАНИЕ}

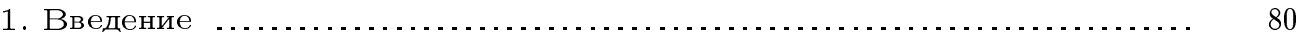

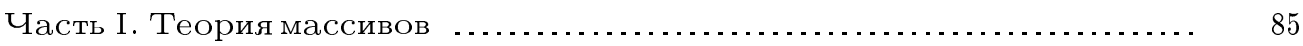

2. Мотивировка и определение массивов ......................... 85

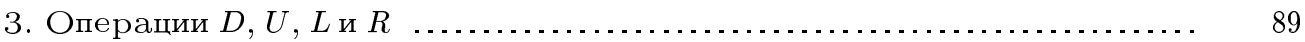

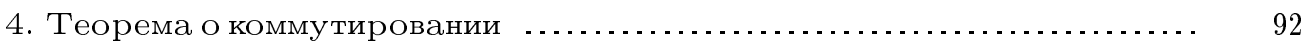

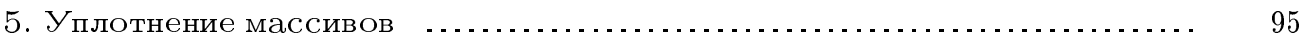

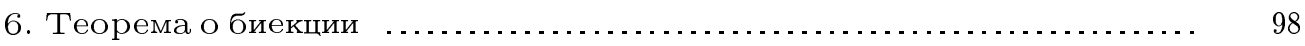

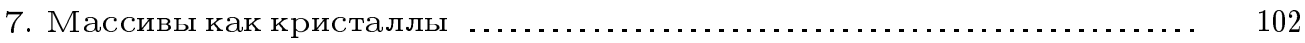

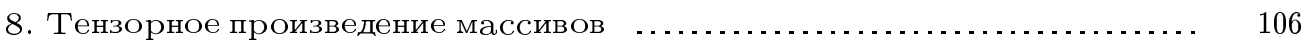

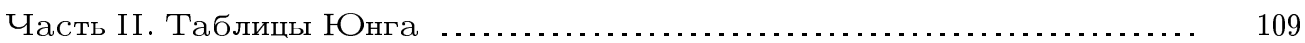

9. Словесное представление массивов ............................. 109

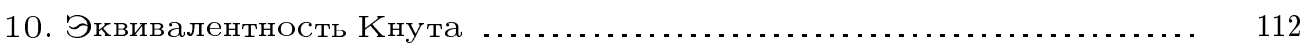

11. Косые таблицы ..................................................... 115

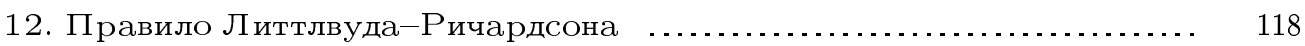

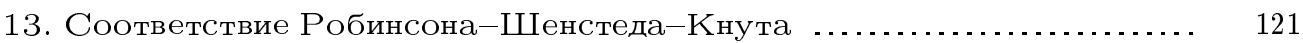

14. Дуальные таблицы и плоские разбиения $\ldots \ldots \ldots \ldots \ldots \ldots \ldots \ldots \ldots \ldots \ldots . . \ldots \ldots$

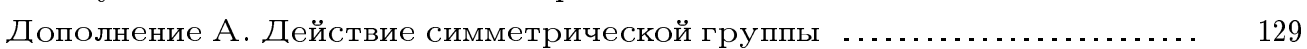

Дополнение В. Связь с частично упорядоченными множествами $\ldots . . . . .131$

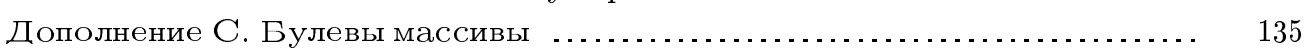

Список литературы ................................................ 140

(C) В. И. ДАнилов, Г. А. Кошевой 2005 


\section{1. Введение}

1.1. В этой работе мы хотим рассказать о таблицах Юнга - объектах, введенных сотню лет назад и оказавшихся довольно удобным и наглядньм средством изложения многих вешей, связанных с теорией представлений, эрмитовыми матрицами, исчислением Шуберта и т. п.; см. [40], [21], [29], [22], [45].

В этой науке имеются два основных понятия: диаграммы Юнга и таблицы Юнга. Диаграмма (или схема) Юнга - это просто несколько поставленных друг на друга рядов квадратиков (яшиков), приставленных друг к другу на манер, изображенный на рис. 1.

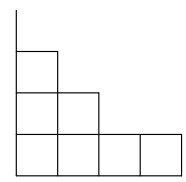

Рис. 1.

Главное - что ящики выровнены по левому краю и что вьшележаший ряд не длиннее нижележашего. (Отметим сразу, что во многих книгах и статьях, особенно на английском и русском языках, эти ряды направляют вниз, а не наверх, как у нас, что соответствует французской традиции.)

Обычно число ящиков в самом нижнем ряду обозначают $\lambda(1)$ (или $\left.\lambda_{1}\right)$, в следуюшем - $\lambda(2)$ и т.д. Так, приведенная вьше диаграмма задает последовательность $\lambda=(4,2,1,0)$. Таким образом, задать диаграмму Юнга - это то же самое, что задать невозрастающую последовательность неотрищательных целых чисел

$$
\lambda=(\lambda(1) \geqslant \cdots \geqslant \lambda(m))
$$

Сумма $|\lambda|=\sum_{k} \lambda(k)$ равняется обшему числу квадратиков в нашей диаграмме. С этой точки зрения диаграмма Юнга - это просто разбиение целого числа $|\lambda|$.

1.2. Почему же вместо разбиений предпочитают пользоваться диаграммами? Для этого есть две причины. Первая - диаграммы более наглядны, в некоторых случаях легче узнаются глазом (см. высказьвание Ю.И. Манина [41] о язьках и наглядных геометрических образах). Вторая - в яшики диаграммы можно помешать некоторые предметы. Пусть $Y$ - это диаграмма Юнга, понимаемая как множество своих ящиков. Тогда заполнение $Y$ элементами из некоторого множества (алфавита) $I$ - это отображение $F: Y \rightarrow I$. Мы пишем $F(i, j)$ для предмета, лежашего в яшике с координатами $(i, j) ; i$ здесь - горизонтальньй номер (номер столбца), а $j$ - вертикальньй (номер строки) рассматриваемого ящика.

Так как $Y$ естественно упорядочено (как подмножество $\mathbb{Z}_{+}^{2}$ ), разумно считать алфавит $I$ тоже упорядоченным множеством и интересоваться монотонньми заполнениями. Обычно в качестве $I$ берут множество целых (или натуральных) чисел. Заполнение $T$ называется полустандартной таблицей Юнга (или просто таблищей Юнга),

Работа частично поддержана грантом НШ-1939.2003.6. Г. А. Кошевой выражает также благодарность за поддержку российско-французской лаборатории LIFR MIIP и Фонду содействия отечественной науке. Авторы благодарят А. М. Вершика, М. Кашивару, Ан. Н. Кириллова, А. Ласку, Ж.-К. Новелли и И. Пака за полезные обсуждения. 
если $T$ нестрого возрастает по $i$ и строго возрастает по $j$. Это центральное понятие теории таблиц Юнга. Стоит сразу упомянуть два близких понятия. Полустандарт-

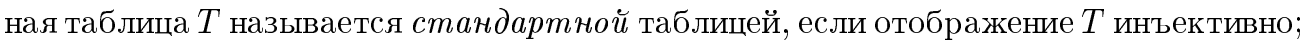
обычно в этом случае для заполнения используют числа от 1 до $|Y|$. Заполнение $F$ называется плоским разбиением, если $F$ нестрого убывает (по $i$ и $j$ ).

Все это можно разньми способами переиначивать. Мы не будем особенно увлекаться этим, как и указанием альтернативной терминологии. Лучше скажем, почему эти понятия представляют интерес.

1.3. Интерес диаграмм или разбиений в том, что они параметризуют разные интересные объекты. С простыми объектами (такими, как множества или векторные пространства) естественно связывать числа. Более сложные объекты часто оказываются связанными с разбиениями.

Представим, что на конечном множестве $X$ имеется некоторое отношение эквивалентности. Числа элементов классов эквивалентности, упорядоченные по невозрастанию, дают некоторое разбиение числа $n=|X|$, или диаграмму с $n$ клетками. Такая ситуация возникает, когда задан автоморфизм $\pi$ конечного множества $X$. Тогда $X$ разбивается на орбиты; мошности этих орбит дают разбиение $|X|$ - так назьваемьй цикловой, или орбитальный, тип перестановки $\pi$. Если мы заменим перестановку $\pi$ на сопряженную, орбитальный тип не изменится. Тем самьм множество классов сопряженных элементов в групе перестановок $S_{n}$ множества из $n$ элементов естественно отождествляется с множеством $\mathbb{Y}_{n}$ диаграмм Юнга с $n$ клетками.

Аналогично можно поступить, если имеется разложение конечномерного векторного пространства в прямую сумму.

Другая похожая ситуация. Пусть $M-$ модуль конечной длины над кольцом дискретного нормирования $A$ с униформизуюшей $t$. Хорошо известно [40], что такой модуль разлагается в прямую сумму циклических модулей $A / t^{l} A$ :

$$
M=\bigoplus_{i}\left(A / t^{l_{i}} A\right)
$$

Само разложение не единственно, но набор чисел $\left(l_{i}\right)$ определен однозначно с точностью до нумерации. Поэтому снова естественно упорядочить эти числа в невозрастающем порядке, и мы получаем разбиение, или диаграмму Юнга.

Эти примеры лежат на поверхности. Но есть и менее очевидные.

1.4. Представления симметрической группь $S_{n}$. Из теории представлений конечных групп известно, что число различных неприводимых представлений равно числу классов сопряженных элементов в групе. Применительно к симметрической группе $S_{n}$ это означает, что для каждой диаграммы Юнга с $n$ клетками (или для каждого разбиения $\lambda$ числа $n$ ) должен существовать свой неприводимый модуль. И действительно, такие модули $S_{\lambda}$ естественно строятся по $\lambda$ и называются модулями Шnехта. Об этом имеется многочисленная литература ([21], [23], [29], [45] - и это только самые последние книги). Размерность представления $S_{\lambda}$ равна числу стандартных таблиц формы $\lambda$. И снова это не просто совпадение чисел: имеется естественньй базис в $S_{\lambda}$, параметризованный стандартными таблицами [21], [43]. Именно при изучении представлений симметрических групп в работах Юнга появились диаграммы и таблищы Юнга. 
1.5. Представления общей линейной группь $G L(n)$. Линейным вариантом множества с $n$ элементами является $n$-мерное векторное пространство (скажем, над полем $\mathbb{C}$ ). Линейньм аналогом группы $S_{n}$ является группа $G L(n)$ всех линейных автоморфизмов такого пространства. Оказьвается, что неприводимые представления этой групшы также тесно связаны с диаграммами и таблицами Юнга. А именно, с каждой диаграммой Юнга, имеюшей не более $n$ строк (или с разбиением $\lambda$, имеющим $n$ частей), можно связать неприводимое представление $V_{\lambda}$ группы $G L(n)[21],[23]$. И снова эта конструкция естественна, по крайней мере в том смысле, что $V_{\lambda}$ обладает базисом, параметризованным полустандартными таблицами формы $\lambda$, заполненньми числами от 1 до $n$. Так получаются не все неприводимые конечномерные представления, а только полиномиальные. Но остальные получаются простой “подкруткой” на степень определителя (или на одномерное представление), так что в каком-то смысле мы получаем все представления.

1.6. Симметрические функиии. Симметрическая группа $S_{n}$ естественно действует на кольце многочленов $\mathbb{Z}\left[x_{1}, \ldots, x_{n}\right]$ от $n$ переменных. Инвариантные относительно этого действия многочлены назьваются симметрическими и образуют подкольцо $\Lambda_{n}$. Моном $x^{m}=x_{1}^{m_{1}} \cdots x_{n}^{m_{n}}$ действием группы можно превратить в такой, что $m_{1} \geqslant \cdots \geqslant m_{n}$. Поэтому естественно ожидать, что в кольце $\Lambda_{n}$ имеется базис, параметризованный разбиениями $\lambda$, имеющими $n$ частей. ${ }^{1}$

Наиболее интересньй базис образуют так называемые $S$-функции или функции (многочлены) Шура, $s_{\lambda}$. Более подробно о них мы расскажем в разделе 12. В частности, произведение двух функций Шура $s_{\mu} s_{\nu}$ является симметрической функцией и поэтому представляется как целочисленная линейная комбинация функций Шура:

$$
s_{\mu} s_{\nu}=\sum_{\lambda} c_{\mu, \nu}^{\lambda} s_{\lambda}
$$

Целые числа $c_{\mu, \nu}^{\lambda}$ известны как коэффициенты Литтлвуда-Ричардсона, поскольку последние предложили некоторое комбинаторное правило для их нахождения (драматическая история этого правила рассказывается в [51]).

Интерес функций Шура связан с тем, что $s_{\lambda}$ является характером неприводимого представления $V_{\lambda}$, рассмотренного в предыдушем пункте. В частности, коэффищиент Литтлвуда-Ричардсона $c_{\mu, \nu}^{\lambda}$ указьвает кратность, с которой представление $V_{\lambda}$ входит в тензорное произведение $V_{\mu} \otimes V_{\nu}$, откуда видно, что $c_{\mu, \nu}^{\lambda}$ - неотрищательны.

Мы видим, что имеется тесная связь между пш. 1.4-1.6. Развив одну из этих теорий (или абстрактную комбинаторику таблиц Юнга), можно уже без особого труда получать факты про остальные теории. В разных книгах выбирается своя последовательность изложения.

1.7. Исчисление Шуберта. Так назьвают обычно теорию пересечений на многообразиях Грассмана, см. [23], [26], [22]. Зафиксируем $n$-мерное комплексное векторное пространство $V$. Обозначим через $G r^{s}(V)=G r_{r}(V)$ множество всех векторных подпространств $W \subset V$ коразмерности $s$ (или размерности $r=n-s$ ). Это множество является гладким проективным алгебраическим многообразием размерности $s r$. Пусть теперь $\lambda=(\lambda(1) \geqslant \cdots \geqslant \lambda(r))$ - некоторое разбиение (или диаграмма Юнга)

\footnotetext{
${ }^{1}$ На самом деле, есть даже несколько таких базисов; взаимоотношения между ними см. в книге И. Макдоналшда [40].
} 
c $r$ частями, причем $\lambda(1) \leqslant s$. Наглядно это означает, что наша диаграмма вписана в прямоугольник с $r$ строками и $s$ столбцами, $\lambda \subset s \times r$ (см. рис. 2).

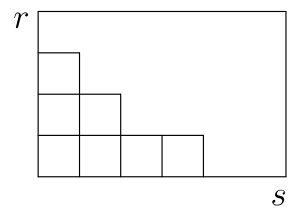

Рис. 2

$\mathrm{C}$ каждьм таким разбиением $\lambda$ можно связать так назьваемьй класс Шуберта $\sigma_{\lambda}$ в кольце когомологий $H^{*}\left(G r^{s}(V), \mathbb{Z}\right)$. Более точно, класс $\sigma_{\lambda}$ лежит в групе $H^{2|\lambda|}\left(G r^{s}(V), \mathbb{Z}\right)$. Например, если $\lambda=(0, \ldots, 0)$, то класс $\sigma_{0}$ есть фундаментальньй класс всего грассманиана. Если $\lambda=(s, \ldots, s)=\left(s^{r}\right)$, мы получаем класс точки в грассманиане.

Оказьвается, что классы Шуберта образуют аддитивньй базис кольца когомологий $H^{*}\left(G r^{s}(V), \mathbb{Z}\right)$. Произведение (в кольце когомологий) классов $\sigma_{\mu} \sigma_{\nu}$ снова представляется как линейная комбинация классов $\sigma_{\lambda}$, причем коэффициентами служат те же числа Литтлвуда-Ричардсона [22].

1.8. Таблицы Юнга участвуют и в других конструкциях, связанных с перестановками и их обобщениями. Наиболее знаменитым является соответствие Робинсона-Шенстеда-Кнута $(R S K)$. Оказывается [46], с любой перестановкой $\pi \in S_{n}$ можно связать пару стандартных таблиц $P(\pi)$ и $Q(\pi)$ одинаковой формы размера $n$. И что замечательно, эта конструкция обратима.

Кнут [34] обобщил эту конструкцию на произвольные неотрицательные матрищы (обобщенные перестановки); стандартные таблицы заменяются при этом полустандартными. Конструкция $R S K$ оставляет странное впечатление. С одной стороны, чувствуется, что она затрагивает какие-то глубокие веши (см., например, работы [2], [15], [32], [49]). С другой стороны, она выглядит как “кухонный рецепт": производи согласно некоторым предписаниям определенные преобразования с таблищами и получишь $P$ и $Q$. Собственно говоря, имеется даже два рецепта (алгоритма) - вставки (бампинга) и скольжения (слайдинга, или јеu de taquin), которые приводят к так называемому плактическому моноиду. Последний уже сравнительно легко позволяет переходить к функциям Шура и доказьвать правило вычисления произведения функций Шура. Все это и составляет стандартное содержание теории таблиц Юнга, см. [21], [45].

1.9. В настояшей работе излагаются некоторые (отнюдь не все!) понятия и результаты комбинаторики таблиц Юнга. Главное новшество заключается в несколько нетрадиционном взгляде на предмет. Мы бо́льшую часть времени будем заниматься не таблицами, а другими объектами, которые мы назьваем массивами и которые фактически являются матрицами. Массивы тесно связаны с таблицами (см. следуюший раздел, а также раздел 9); более точно, таблицы Юнга - это фактически $\mathbf{D}$-плотные массивы.

Мотивировка введения более общего объекта следуюшая. Упомянутые вьше операции-алгоритмы с таблицами состоят из ряда промежуточных шагов, и на этих этапах преобразуемый объект не является таблицей. Только на последнем шаге он снова 
становится таблицей. Это доставляет понятные технические неудобства. Одно из преимуществ массивов в том, что они имеют смысл и на промежуточных этапах.

Более точно, мы вводим серию “элементарных" операций над массивами, $D, U, L$ и $R$ (вниз, вверх, влево, вправо). Классические операции бампинга и слайдинга представляются как некоторые комплексы этих элементарных операций. "Вертикальные" операции $D$ и $U$ в некотором смысле почти обратны друг к другу; это же верно для “горизонтальных" операций $L$ и $R$ (см. предложение 3.5$)$. Центральный факт (теорема 4.2) состоит в том, что вертикальные и горизонтальные операции коммутируют между собой. Следствиями этого факта является независимость многих конструкций от произвола на промежуточных шагах (см. раздел 5). Другим важным следствием является теорема о биекции из раздела 6 , вариант соответствия $R S K$.

Стоит отметить еще одну черту массивов. Если рассматривать только вертикальные операции $D$ и $U$, мы получим некую пародию на представления групш $G L$. Например, имеется аналог леммы Шура (теорема 7.9). Более точно можно сказать, что массивы образуют кристалл в терминологии Кашивары. Подробнее об этом говорится в разделах 7 и 8. В частности, можно говорить о тензорных произведениях, разложениях на неприводимые и т. п. Стоит отметить одно преимущество массивов по сравнению с кристаллами или представлениями. Оно состоит в том, что даже изоморфные "неприводимые компоненты" индивидуализированы, каждая имеет свою "метку" (или индекс), выраженную D-плотным массивом. Это позволяет говорить не просто о числе неприводимых компонент, но о мно сестве. В разделе 8 мы останавливаемся на двух биекциях между такими множествами, связанных с ассоциативностью и коммутативностью тензорных произведений. Похожие вопросы с иных позиций рассматривались в [36], [28].

1.10. В части II мы показьваем, как основные результаты комбинаторики таблищ Юнга уже сравнительно просто и единообразно получаются из теории массивов. Мы кратко останавливаемся на связи наших операций $D$ и $U$ с традиционной техникой - бампингом Шенстеда-Кнута и јеu de taquin Шютценберже, а также понятием плактического моноида Ласку-Шютценберже. Обсуждаем правило Литтлвуда-Ричардсона, столь долго стимулировавшего развитие теории таблиц Юнга, а также соответствие $R S K$.

В разделе 14 устанавливается связь таблиц Юнга с плоскими разбиениями, или, что то же самое, с трехмерными вариантами диаграмм Юнга.

В дополнениях обсуждаются три более специальных результатапро массивы (действия симметрических груп на массивы, связь с частично упорядоченными множествами, теория булевых массивов).

1.11. Хотя понятие массива, насколько нам известно, оригинально, оно не является чем-то принципиально новым. Массивы в форме матриц встречаются уже у Кнута [34], хотя и без операций. Массивы как слова изучаются под названием плактического моноида. Правда, словесный подход страдает однобокостью. Кроме того, вместо операций там работают с менее информативньм понятием эквивалентности по Кнуту.

Мы бы хотели подчеркнуть два преимушества массивного подхода. Во-первых, табличньй и словесньй подходы страдают однобокостью. При этом подходе массивы читаются как тексты в горизонтальном алфавите $I$. Это нарушает изначальную симметрию и равноправие двух алфавитов $I$ и $J$. Какие-то понятия на одностороннем языке выглядят красиво или привычно. Но дуальные понятия и конструкции звучат 
уже диссонансом. В любом случае, практически все конструкции теории таблиц Юнга прекрасно выражаются на языке массивов. И второе, уже менее принципиальное преимущество. Массивньй подход изначально открыт для вешественных чисел, а не только для натуральных, тогда как клеточная структура диаграмм (или слов) приемлет лишь целые количества. В частности, многие естественные множества массивов реализуются точками вьпуклых многогранников (а целочисленные массивы - как целые точки в этих многогранниках). Стоит, правда, предостеречь, что интересные отображения между этим многогранниками часто оказываются не линейньми, но лишш кусочно линейными.

1.12. Недавно появился еще один интересный подход к предмету нашего обзора, о котором мы лишш кратко упомянем (см. также пп. 2.8 и 8.7). С этой стороны мы интересуемся функциями, заданньми на некоторых специальных подмножествах (гридах) в $\mathbb{Z}^{2}$. Функции тоже не произвольные, но обладают в той или иной мере свойствами “дискретной вогнутости”. Истоки этого подхода можно найти в понятиях паттерна Гельфанда-Цетлина и треугольников Беренштейна-Зелевинского [5]. Более точная формулировка была найдена У. Фултоном (см. [8]): таблица Литтлвуда-Ричардсона - это то же самое, что (целочисленная) дискретно вогнутая функция (или улей в терминологии [35], [36]). Авторы независимо пришли к этому наблюдению, занимаясь развитием общей теории дискретной вьпуклости [13]. Этот функциональньй подход с успехом применялся к задачам о спектрах эрмитовых матриц [35], [11] и о нильпотентных операторах [10]. Роль операции $\mathbf{D}$ для массивов (или jeu de taquin - для таблиц Юнга) играет так назьваемая октаэдральная рекурсия (см. [20], [36], [28]).

\section{Часть I. Теория массивов}

\section{2. Мотивировка и определение массивов}

2.1. Напомним, что таблица Юнга - это диаграмма Юнга, заполненная обычно числами $1,2, \ldots$, причем числа возрастают (нестрого) в каждой строке и (строго) по вертикали. Начнем с простейшего случая, когда имеется всего одна строка. Например,

\begin{tabular}{|l|l|l|l|l|l|}
\hline 1 & 1 & 3 & 3 & 3 & 4 \\
\hline
\end{tabular}

Видно, что значение имеет только порядок между числами. Эти числа никогда не складьвают, но только сравнивают. То есть символы внутри клеток означают не количества, но качества. И вместо чисел можно брать элементы (символы) из любого алфавита $I$, т.е. линейно упорядоченного множества. Если понимать элементы алфавита $I$ как буквы, то однострочная таблица - это просто слово в алфавите. Но не произвольное - буквы должны идти в неубывающем порядке. Так, предыдушая таблица в алфавите $a, b, c, d$ вьглядит как слово $a a c c c d$. Чтобы сократить эту запись (напоминаюшую изображение чисел как последовательности палочек), можно писать $a^{2} c^{3} d$ или, чуть более педантично, $a^{2} b^{0} c^{3} d^{1}$.

Таким образом, однострочную таблицу можно восстановить, зная только набор показателей $(2,0,3,1)$. В общем случае задать однострочную таблицу с алфавитом $I-$ то же самое, что задать отображение $a: I \rightarrow \mathbb{Z}_{+}$. (Для простоты мы будем предполагать алфавит $I$ конечным и равным $\{1, \ldots, n\}$; в обшем случае надо считать, что $a$ имеет конечный носитель.) Такое отображение, т.е. попросту вектор, мы и называем 
(однострочньмм) массивом. Сразу надо сказать, что ограничиваться массивами с целыми значениями совсем необязательно; можно допускать любые неотрицательные значения.

Массив, как и таблицу, удобно помещать в клетки. Нарисуем подряд $n$ клеток, и в клетку с номером $i$ поместим "массу" величины $a(i)$. Так, строка выше представляется как

\begin{tabular}{|l|l|l|l|}
\hline 2 & 0 & 3 & 1 \\
\hline
\end{tabular}

Когда эта масса целая, удобно считать, что в клетке-яшике $i$ лежит $a(i)$ шариков единичной массы. Внешне такой массив похож на таблицу, но стоит подчеркнуть два отличия. Во-первых, массив имеет фиксированное (равное $n$ ) число клеток. Во-вторых, в ящиках располагаются теперь количества.

В общем случае и таблица, и массив могут иметь несколько строк. Чтобы говорить об этом, нужно обзавестись еше одним, вертикальным алфавитом $J$ (обычно мы считаем, что $J=\{1, \ldots, m\})$. Так мы приходим к общему определению.

2.2. ОПРЕДЕЛЕНИЕ. Массивом (точнее, массивом формата $I \times J)$ назьвается семейство $a=(a(i, j), i \in I, j \in J)$ неотрицательных чисел.

Иначе говоря, массив - это отображение $a: I \times J \rightarrow \mathbb{R}_{+}$, т.е., попросту говоря, матрица с неотрицательными коэффициентами. Мы, однако, не хотим назьвать эти объекты матрицами. Матрицы естественно складывать и перемножать. Мы никогда не будем этого делать с массивами. Напротив, мы введем в следующем разделе серию операций, которые будем применять к массивам. Эти операции берут часть массы в некоторой клетке $(i, j)$ и переносят ее в соседнюю клетку.

Другая, менее серьезная причина, чтобы не назьвать массивы матрицами, состоит в том, что мы располагаем числа $a(i, j)$, пользуясь декартовой системой. То есть число $a(2,3)$ помешается в клетке, находящейся на пересечении второго столбца и третьей строки. Причем строки нумеруются снизу вверх. (В матричных обозначениях это число стояло бы во второй - считая сверху - строке и третьем столбце.)

Для облегчения понимания читатель может считать (и мы будем это делать в примерах), что числа $a(i, j)$ - целые неотрицательные. Так проще следить за последующими конструкциями. Переход к дробным массивам не представляет уже ничего сушественно нового.

Множество всех массивов обозначается $\mathscr{A}(I, J)$. Мы будем писать $\mathscr{A}_{\mathbb{Z}}$, если хотим подчеркнуть, что рассматриваем целочисленные массивы.

2.3. Еще несколько простых понятий, относяшихся к массивам. При фиксированном $i \in I$ семейство чисел $a(i, j), j \in J$, назьвается $i$-м столбиом массива $a$. Сумма $a(i, J)=\sum_{j \in J} a(i, j)$ всех элементов $i$-го столбца назьвается $i$-й столбиовой суммой. Вектор всех столбцовых сумм $a(\cdot, J)$ мы будем называть также $I$-весом массива $a$ и обозначать $w t_{I}(a)$; это элемент векторного пространства $\mathbb{R} \otimes I$. Симметрично определяются строки и строчные суммы; вектор строчных сумм $a(I, \cdot)$ назьвается $J$-весом и обозначается $w t_{J}(a)$.

Меняя $I$ и $J$ местами, мы транспонируем массив, т.е. получаем $(J \times I)$-массив $a^{T}$, $a^{T}(j, i)=a(i, j)$.

Есть еше одна интересная операция над массивами: центральная симметрия (здесь приходится считать множества $I$ и $J$ конечными). Для массива $a$ через $* a$ обозначается массив того же формата, причем $* a(i, j)=a(n+1-i, m+1-j)$. 
2.4. Как уже говорилось вьше, каждая таблица (с $J$ строками и алфавитом $I$ ) определяет соответствуюший массив формата $I \times J$. Конечно, эта таблица однозначно определяется своим массивом. Однако из таблиц получаются не любые массивы. Например, если мы попробуем восстановить “таблицу” по массиву

\begin{tabular}{|l|l|l|l|}
\hline 1 & 1 & 3 & 2 \\
\hline 2 & 0 & 3 & 1 \\
\hline
\end{tabular}

мы получим две строки

$$
\begin{array}{lllllll}
1 & 2 & 3 & 3 & 3 & 4 & 4 \\
1 & 1 & 3 & 3 & 3 & 4 &
\end{array},
$$

которые не образуют таблицу Юнга. Иначе говоря, таблицы Юнга дают некоторые специальные массивы. Нетрудно написать соответствуюшие условия. Так как эти условия связьвают две соседние строки, мы можем ограничиться здесь таблицами и массивами из двух строк.

2.5. ЛеммА. Двухстрочный иелочисленный массив $a(i, j), j=1,2$, происходит из полустандартной таблиць Юнга тогда и только тогда, когда для любого $k \in I$ выполнены неравенства

$$
a(1,2)+\cdots+a(k, 2) \leqslant a(1,1)+\cdots+a(k-1,1) .
$$

ДокАЗАТЕльство. Напишем по массиву а соответствуюшую “таблицу”. На первых $a(1,1)+\cdots+a(k-1,1)$ местах нижнего ряда этой таблицы стоят символы от 1 до $k-1$. Аналогично для верхнего ряда. Поэтому если символ $k$ находится в верхнем ряду, он стоит на месте с номером $\leqslant a(1,2)+\cdots+a(k, 2)$. В силу неравенства $(*)$ под ним стоит символ, строго меньший, чем $k$.

Рассуждение в обратную сторону аналогично.

Массив, удовлетворяющий неравенствам (*) (во всех соседних строках), мы называем $\mathbf{D}$-nлотнылм или плотным вниз. Таким образом, из леммы мы получаем описание тех массивов, которые получаются из полустандартных таблиц: это $\mathbf{D}$-плотные и целочисленные массивы.

Приведем оправдание терминологии в случае целочисленного массива; позже такая интерпретация будет очень полезна. Довольно легко понять, что неравенства (*) означают, что для каждого шара из второго ряда имеется "свой” шар в нижнем ряду, которьй расположен строго левее. То есть каждьй шар из второго ряда имеет "свою опору" в нижележашем ряду, которая не дает ему опуститься. И в этом смысле шары в массиве расположены "плотно".

2.6. Основная конструкция теории массивов состоит в том, что неплотные массивы можно уплотнять, делая их все более плотным. Чтобы объяснить происходящее, рассмотрим простой двухстрочньй массив из п. 2.4. Ему соответствует “таблица"

$$
\begin{array}{lllllll}
1 & 2 & 3 & 3 & 3 & 4 & 4 \\
1 & 1 & 3 & 3 & 3 & 4 &
\end{array}
$$


Если мы сдвинем на три (или более) позиций нижнюю строку, мы получим то, что в теории таблиц называют косой таблицей

$$
\begin{array}{lllllllll}
1 & 2 & 3 & 3 & 3 & 4 & 4 & & \\
* & * & * & 1 & 1 & 3 & 3 & 3 & 4
\end{array} .
$$

То есть мы восстановили полустандартность (строгое возрастание по вертикали), но за счет добавления фиктивных пустых (у нас они помечены как *) клеточек. Теперь, пользуясь jeu de taquin, игрой, придуманной Шютценберже, мы можем одну за другой “вьгнать” наружу пустые клетки. В этой игре пустая клетка меняется местами со своим соседом справа или сверху. Если при этом у нее два соседа, она меняется местами с меньшим соседом; если оба соседа одинаковы, она меняется с верхним соседом. Легко проверить, что после вытеснения всех пустых клеток останется таблища

$$
\begin{array}{lllllllll}
2 & 3 & 4 & 4 & & & & & \\
1 & 1 & 1 & 3 & 3 & 3 & 3 & 3 & 4
\end{array}
$$

Чтобы понять это преобразование на языке массивов, удобнее чуть иначе взглянуть на эту игру. Давайте передвинем нижнюю строку, но не как целое, а с разрывами. Возьмем в нижней строке клетку с символом 1. Сдвинем нижние клетки так, чтобы над 1 стало стоять большее число. В нашем примере надо сдвинуть на одно место. Потом перейдем к клеткам с символом 2; у нас таких нет. Потом к клеткам с символом три. Их (и все следующие за ними) приходится сдвинуть еше на два места. И так далее. В результате мы получим “таблицу" с рваной нижней строкой

\begin{tabular}{|l|l|l|l|l|l|l|l|l|}
\hline 1 & 2 & 3 & 3 & 3 & 4 & 4 & \multicolumn{3}{|c|}{} \\
\hline & 1 & 1 & & & 3 & 3 & 3 & 4 \\
\hline
\end{tabular}

Теперь опускаем на пустые места клетки из второго ряда:

\begin{tabular}{|l|l|l|l|l|l|l|l|l|}
\hline & 2 & 3 & & & 4 & 4 & \multicolumn{2}{|l}{} \\
\hline 1 & 1 & 1 & 3 & 3 & 3 & 3 & 3 & 4 \\
\hline
\end{tabular}

и уплотняем влево верхний ряд, ликвидируя разрывы. Получаем ту же таблицу

$$
\begin{array}{llllllllll}
2 & 3 & 4 & 4 & & & & & \\
1 & 1 & 1 & 3 & 3 & 3 & 3 & 3 & 4
\end{array} \text {. }
$$

2.7. Переводя сказанное вьше на язык массивов и шаров, мы получаем такую картину. Двигаясь слева направо по верхнему ряду, мы ишем для каждого шара подходящего партнера (внизу и слева). Некоторые шары (в нашем примере, шар из клетки $(1,2)$ и два шара из клетки $(3,2))$ не находят поддержки и висят “свободно". Рецепт состоит в том, чтобы опустить вниз эти свободные шары. В результате мы получаем D-плотньй массив

\begin{tabular}{|l|l|l|l|}
\hline 0 & 1 & 1 & 2 \\
\hline 3 & 0 & 5 & 1 \\
\hline
\end{tabular}


Можно не опускать сразу все свободные шары, а делать это постепенно. При этом, как мы увидим, начинать надо с самого правого верхнего свободного шара. Эту операцию мы обозначим как $D$. Можно пойти еше дальше и переносить вниз не единичную массу, а лишш ее порцию $t$. Такую операцию естественно обозначать как $D^{t}$. Более обстоятельно эти операции обсуждаются в следуюшем разделе.

2.8. Как мы увидим, массивы тесно связаны не только с таблицами, но также со словами-текстами и частично упорядоченньми множествами (посетами). В разделах 9-11 и дополнении В мы расскажем об этом более подробно. Наконец, как мы упомянули во введении, массивы связаны с функциями на прямоугольных гридах $\{0,1, \ldots, n\} \times\{0,1, \ldots, m\}$. Скажем об этом кратко.

Пусть $a$ - массив. Свяжем с ним функцию $f=f_{a}$, определенную на множестве $\{0,1, \ldots, n\} \times\{0,1, \ldots, m\}$, с помошю следующей формулы:

$$
f(i, j)=\sum a\left(i^{\prime}, j^{\prime}\right)
$$

где $i^{\prime} \leqslant i, j^{\prime} \leqslant j$. Если $i=0$, то в сумме нет слагаемых и мы полагаем ее равной нулю. Аналогично для $j=0$. Если же $i$ и $j$ больше нуля, то

$$
f(i, j)-f(i-1, j)-f(i, j-1)+f(i-1, j-1)=a(i, j) .
$$

Так как $a(i, j) \geqslant 0$, функция $f$ супермодулярна, т.е. левая часть (разностньй аналог смешанной частной производной $\left.\partial^{2} f / \partial x \partial y\right)$ неотрицательна. Верно и обратное: если нам дана супермодулярная функция $f$ на $\{0,1, \ldots, n\} \times\{0,1, \ldots, m\}$, мы с помошњю предыдущей формулы получаем массив $a$.

Массив $a$ является $\mathbf{D}$-плотным тогда и только тогда, когда функция $f$ обладает дополнительным свойством “вогнутости”, а именно, когда вьполняются неравенства

$$
f(i+1, j)+f(i, j)-f(i, j-1)-f(i+1, j+1) \geqslant 0 .
$$

\section{3. Операции $D, U, L$ и $R$}

3.1. На массивах формата $I \times J$ мы введем четыре серии операций $D_{j}, U_{j}(j=$ $1, \ldots, m-1)$ и $L_{i}, R_{i}(i=1, \ldots, n-1)$. Операция $D_{j}$ перемещает (быть может) некоторьй шар из $(j+1)$-й строки в $j$-ю строку, сохраняя расположение шара в том же столбце. То есть она спускает шар вертикально вниз на один этаж, откуда и обозначение $D$ (down). Операция $U_{j}$, напротив, перемешает шар вертикально вверх, из $j$-й строки в $(j+1)$-ю. Операция $L_{i}$ двигает некоторый шар влево, а $R_{i}$ - вправо. Все они определяются однотипно, поэтому мы ограничимся определением только операции $D_{j}$. Более того, действие $D_{j}$ (как и $U_{j}$ ) зависит только от строения массива в строчках $j$ и $j+1$. Поэтому мы можем далее считать, что наш массив состоит ровно из двух строчек - нижней $a(\cdot, 1)$ и верхней $a(\cdot, 2)$. Вместо $D_{1}$ и $U_{1}$ мы будем говорить про $D$ и $U$.

Чтобы определить действие $D$, мы должны сказать, из какой клетки $(i, 2)$ нужно взять шар и переместить его вниз. В п. 2.6 мы уже объяснили на примере, как это делать. Повторим это теперь в общем случае.

3.2. Мы должны поделить все шары верхнего ряда на “имеющие опору" и "свободные, висяшие без поддержки”. Обратимся к яшику $(i, 2)$, в котором находится $a(i, 2)$ 
шаров. Обозначим через $a^{\prime}(i, 2)$ количество свободных шаров и через $a^{\prime \prime}(i, 2)$ - число связанных шаров в этом яшике. Ясно, что $a=a^{\prime}+a^{\prime \prime}$. В первом ряду левее места $i$ находится $A=a(1,1)+\cdots+a(i-1,1)$ шаров; среди них $A^{\prime \prime}=a^{\prime \prime}(1,2)+\cdots+$ $a^{\prime \prime}(i-1,2)$ уже связаны с верхними шарами. Если оставшихся шаров $A-A^{\prime \prime}$ хватит на $a(i, 2)$, то все шары в ящике $(i, 2)$ будут связанньми. Если нет, в этом ящике останется $a^{\prime}(i, 2)=\max \left(0, a(i, 2)-A+A^{\prime \prime}\right)$ свободных шаров. Так шаг за шагом, рекуррентно, мы определяем числа $a^{\prime}(i, 2)$, начиная с $a^{\prime}(1,2)=a(1,2)$.

Можно чуть преобразовать. Обозначим через

$$
\beta_{i}=a(1,2)+\cdots+a(i, 2)-a(1,1)-\ldots-a(i-1,1) .
$$

Тогда

$$
a^{\prime}(i, 2)=\max \left(0, \beta_{i}-\left(a^{\prime}(1,2)+\cdots+a^{\prime}(i-1,2)\right)\right) .
$$

Или

$a^{\prime}(1,2)+\cdots+a^{\prime}(i, 2)=\max \left(a^{\prime}(1,2)+\cdots+a^{\prime}(i-1,2), \beta_{i}\right)=\max \left(\beta_{1}, \ldots, \beta_{i}\right) .(* * *)$

Итак, мы поделили верхние шары на имеющие опору и свободные. Теперь можно определить действие операции $D$. Мы должны взять самьй правьй свободньй шар и переместить его в нижний ряд. Если свободных шаров нет, операция $D$ не меняет массив.

Именно потому, что мы опускали самьй правый свободный шар, он, опустившись вниз, не может стать чьей-то поддержкой (правее его нет свободных шаров). Поэтому повторное действие операции $D$ опускает следующий самый правый свободный шар. И так далее. Так что можно сразу сказать, как вьглядит $k$-я степень $D^{k}$ этого действия: нужно взять $k$ самых правых свободных шаров и опустить их вниз. Если свободных шаров меньше $k$, нужно опустить сколько есть.

Неявно мы предполагали вьше, что массив целочисленньй. Но теперь видно, что это совершенно неважно. Пусть массив произвольньй и $t \geqslant 0$-некоторое вешественное число. Тогда действие $D^{t}-t$-й степени оператора $D$ - состоит в том, что мы берем самую правую свободную массу в количестве $t$ ( а если вся свободная масса $\leqslant t$, то просто всю) и опускаем ее вертикально вниз на первый этаж. Количество свободной массы $a^{\prime}(i, 2)$ в клетке $(i, 2)$ задается формулой $(* * *)$.

Видно, что действие полугруппы $\left(D^{t}, t \geqslant 0\right)$ кусочно линейное. Пока мы перемещаем массу из одной клетки, все линейно. Но когда свободная масса в клетке $(i, 2)$ кончается, мы должны перейти в клетку $(i-1,2)$. И это вызьвает смену линейного режима. Например, если $t$ больше, чем $\max \left(\beta_{1}, \ldots, \beta_{n}\right)$, траектория $D^{t} a$ останавливается.

Кстати, число $\delta=\delta(a)=\max \left(\beta_{1}, \ldots, \beta_{n}\right)$ можно назвать дефектом нашего двухстрочного массива. Если бы левее нашего массива на первом этаже было столько шаров, то этот "расширенный” массив был бы плотным вниз. Но так как их нет, нам нужно применить $D^{\delta(A)}$, чтобы сделать наш массив плотньм. Легко понять, что применение $D^{t}$ уменьшает дефект массива на $t$ (если, конечно, $t \leqslant \delta(a)$ ). Так что, образно говоря, массив $a$ отстоит от своего уплотнения $D^{\delta(a)} a$ на расстоянье $\delta(a)$.

3.3. Аналогично определяется действие $U$. Более формально, оно определяется как сопряжение $D$ с помощью переворачивания * (см. п. 2.3),

$$
U(a)=* D(* a) .
$$


А содержательно, мы должны искать для нижних шаров партнеров на втором этаже (причем расположенных строго правее); шары, не нашедшие партнеров, объявляются свободньми. И операция $U$ поднимает вверх самьй левый свободный нижний шар (если таковой найдется).

Аналогично введенному выше понятию дефекта можно ввести понятие эксцесса. (избытка) $\varepsilon, \varepsilon(a)=\delta(* a)$. Эксцесс $\varepsilon(a)$ указывает расстояние от $a$ до его уплотнения вверх. Можно проверить, что $\varepsilon(a)+\delta(a)=w_{2}(a)-w_{1}(a)$, где $w_{j}(a)=a(I, j), j=1,2$.

Главное свойство операций $D$ и $U$ состоит в том, что они “почти" обратны друг к другу. Они не могут быть в точности обратными друг к другу, и причина проста. Например, если массив $a$ плотньй вниз, то операция $D$ оставляет его на месте, $D a=a$. В то время как операция $U$ вполне может действовать нетривиально. И в этом случае $U D a=U a \neq a$.

Однако если $D$ действует на $a$, то $U D a=a$. Дело в том, что когда $D$ опускает некоторьй шар, то $U$ “этот же” шар поднимает обратно. Чтобы убедиться в этом, нам нужно более аккуратно производить спаривание, или матчинг, верхних и нижних шаров.

3.4. Стабильный матчинг. Мы не хотим отвлекать читателя точным определением, что такое стабильньй матчинг. Вместо этого укажем процедуру его организации. Будем перебирать шары верхнего ряда один за другим, двигаясь по яшиками слева направо. (Фактически, мы разворачиваем верхнюю строчку массива как слово в таблище.) Взяв очередной шар $b$, мы назначаем ему партнером самый правьй шар с первого этажа, которьй а) расположен левее $b$, и b) не является партнером других, более ранних шаров. Если такого нет, шар $b$ объявляется свободным. И мы переходим к следуюшему шару $b^{\prime}$.

В результате мы организуем паросочетание (матчинг) части верхних шаров с частью нижних. Оставшиеся неспаренными шары (как на втором этаже, так и на первом) объявляются свободными. Важно, что верхние свободные шары лежат (нестрого) левее нижних свободных шаров. Так что после удаления связанных пар картина выглядит так:

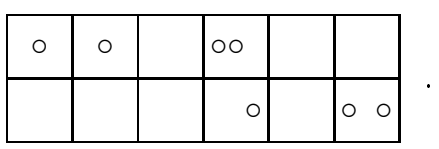

Операция $D$, как уже говорилось, опускает самый правый свободньй верхний шар. Операция $U$ поднимает самый левьй свободный нижний шар. Опущенньй операцией $D$ самьй правьй свободньй верхний шар $b$ становится самым левым свободным нижним. Поэтому под действием $U$ он возвращается на исходное место. То есть $U D a=a$. Если только операция $D$ действительно опускала некоторьй шар.

В предыдущем рассуждении мы неявно предполагали целочисленность массива. В обшем случае мы получаем следующее предложение.

3.5. ПРЕДЛОЖЕНИЕ. Пусть $t \geqslant 0$ таково, что для любого $t^{\prime}<t$ выполнено $D^{t^{\prime}} a \neq D^{t} a$. Тогда $U^{t} D^{t} a=a$.

Конечно, аналогичное утверждение верно и для $D U$.

3.6. Общий случай. Вьше мы определили операции $D$ и $U$ для массива из двух строк. Пусть теперь $a$ - массив с $m$ строчками. Чтобы задать действие, скажем, $D_{j}$, нужно взять строки $j$ и $j+1$ и применить операцию $D$ к этому двухстрочному массиву. 
Аналогично задается действие $U_{j}$. Операции $L_{i}$ определяются как сопряжение $D_{i}$ с помощью транспонирования массива: $L_{i} a=\left(D_{i} a^{T}\right)^{T}$. Аналогично для $R_{i}: R_{i} a=$ $\left(U_{i} a^{T}\right)^{T}$.

Ясно, что операшии $D_{j}$ и $U_{j}$ почти обратны друг к другу, как и $L_{i}$ и $R_{i}$. Кроме того, имеют место следуюшие очевидные соотношения:

$$
D_{j}(* a)=*\left(U_{m-j} a\right), \quad L_{i}(* a)=*\left(R_{n-i} a\right) .
$$

Заметим, наконец, что $D_{j}$ (или любая его степень) не меняет $I$-вес массива, т.е. $w t_{I}\left(D_{j} a\right)=w t_{I}(a)$ для любых $a$ и $j$. Аналогично горизонтальные операции $L$ и $R$ не меняют вертикальный вес $w t_{J}$.

\section{4. Теорема о коммутировании}

4.1. Центральное утверждение про введенные вьше операции состоит в том, что “вертикальные” операции $D_{j}$ и $U_{j}$ (а также любые их степени) коммутируют с “горизонтальными" операциями $L_{i}$ и $R_{i}$.

Убедимся в этом на примере - см. рис. 3.

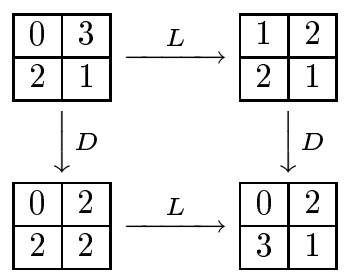

Рис. 3

4.2. ТЕОРемА. Любая степень оператора $L_{i}$ коммутирует с любой степенью onepamopa $D_{j}$.

Тут мы для краткости понимаем $R_{i}$ как $L_{i}^{-1}$ и аналогично для $U_{j}$.

4.3. ДокАЗАТЕльство. Докажем сначала, что оператор $L_{i}$ коммутирует с $D_{j}$, предполагая дополнительно, что массив $а$ целочисленный. (Общий случай сводится к этому в п. 4.5.) Для краткости обозначим $D=D_{j}$ и $L=L_{i}$. Строку $j$ назовем нижней, строку $j+1$ - верхней, аналогично понимаем левьй и правый столбец. Нам нужно показать, что $D L a=L D a$.

Предположение целочисленности позволяет пользоваться ящично-шариковым представлением массива. Чтобы подействовать операцией $D$ на массив $a$, мы должны организовать матчинг $\mu$ между шарами из верхней и нижней строки, взять самьй правьй свободный шар $b$ верхней строки (если он есть) и опустить его вниз. Аналогично действует $L$ : нужно образовать матчинг $\nu$ между правым и левьм столбцами, взять самый верхний свободный шар $b^{\prime}$ справа и переместить его влево.

Действуя операцией $L$ на $a$, мы перемешаем шар $b^{\prime}$ влево. Если мы теперь хотим подействовать операцией $D$ на $L a$, нам нужно пересчитать матчинг $\mu$ для нового массива $L a$. Ключевое наблюдение состоит в том, что матчинг фактически не меняется. Шар $b$, который нужно опустить вниз в массиве $a$, нужно опускать вниз и в массиве $L a$. 

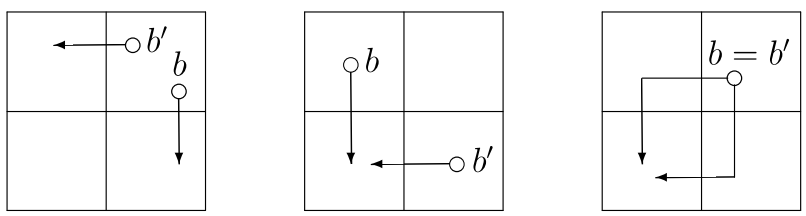

Рис. 4.

Это верно и в том случае, когда $b=b^{\prime}$, и в том случае, когда $b$ (или $b^{\prime}$ ) отсутствует. На рис. 4 изображено несколько вариантов того, что может случиться.

Итак, мы свели все к следующему ключевому утверждению: применение операиии $L$ не меняет матчинг между верхней и нижней строчкой. Конечно, аналогичное утверждение имеет место и в отношении матчинга между правьм и левьм столбцом при действии $D$.

4.4. Доказательство ключевого утверждения. Утверждение, очевидно, верно в двух простых случаях:

a) когда $L$ тривиально действует на массив $a$ и

b) когда передвигаемьй операцией $L$ шар $b^{\prime}$ находится не в интересующих нас верхней и нижней строках.

В самом деле, в этих случаях расположение шаров в интересуюших нас строках совсем не меняется. Поэтому остается рассмотреть два случая - когда шар $b^{\prime}$ находится в нижней строке и когда в верхней.

с) Шар $b^{\prime}$ в нижней строке, т.е. в юго-восточной клетке Ю-В (см. рис. 5).

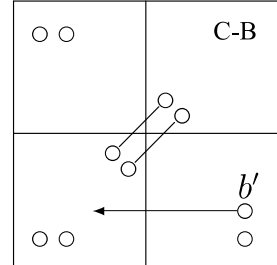

Рис. 5.

Заметим, что все шары, находящиеся в клетке C-B, имеют партнеров (при матчинге $\nu$ ) в клетке Ю-З. В самом деле, так как $b^{\prime}$ - самьй верхний свободный шар в правом столбце, то любой шар в клетке С-В имеет партнера слева и ниже. Этот партнер не может находиться ниже Ю-З, потому что в этом случае его перехватил бы шар $b^{\prime}$.

Но раз так, то всем шарам в клетке С-В в матчинге $\mu$ можно назначить тех же партнеров, что и в матчинге $\nu$. Теперь подействуем операцией $L$ и переместим шар $b^{\prime}$ влево. Видно, что если мы оставим старый матчинг $\mu$, он будет стабильным и в новой ситуации.

d) Шар $b^{\prime}$ в верхней строке, т.е. в клетке С-В (см. рис. 6).

Заметим теперь, что все шары в клетке Ю-З имеют партнеров (при матчинге $\nu$ ) в клетке С-В. В самом деле, в противном случае такой шар мог бы стать партнером шара $b^{\prime}$, что противоречило бы тому, что $b^{\prime}$ свободный. 


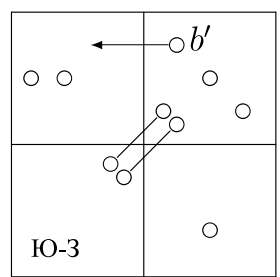

Рис. 6.

Снова в матчинге $\mu$ назначим шарам из клетки Ю-З тех же партнеров, что в матчинге $\nu$. Теперь подействуем операцией $L$ и переместим шар $b^{\prime}$ влево. Снова видно, что старый матчинг $\mu$ останется стабильным и в новой ситуации. Если у $b^{\prime}$ был партнер (в $\mu$ ), то он был в клетке строго левее Ю-З. Если партнера не было, он тем более не появится.

Итак, ключевое утверждение доказано, а вместе с ним и частный случай теоремы.

4.5. Общий случай. Если $D_{j}$ и $L_{i}$ коммутируют, то коммутируют и любые их целые степени. Случай рациональных массивов и рациональных степеней получается простым дроблением шаров единичной массы на более мелкие шарики. Обший случай сводится к рациональному из соображений непрерьвности (и даже липшицевости) наших операторов. Теорема 4.2 доказана.

4.6. В заключение скажем несколько слов о коммутировании $D_{j}$ и $D_{j^{\prime}}$.

Конечно, $D_{j}$ и $D_{j^{\prime}}$ (как и любые их степени) коммутируют между собой, если $j$ и $j^{\prime}$ не соседние символы. Что касается $D_{j}$ и $D_{j+1}$, то они в общем случае не коммутируют. Причина проста: перемешение шара с этажа $j+2$ на этаж $j+1$ может изменить стабильньй матчинг между этажами $j+1$ и $j$.

Тем не менее интересен вопрос о соотношениях между “соседними" операциями $D_{i}$ и $D_{i+1}$. (Конечно, мы можем здесь считать $i=1$, а сам массив имеюшим три строчки.) Например, верно ли соотношение $D_{1}^{\alpha} D_{2}^{\alpha+\beta} D_{1}^{\beta}=D_{2}^{\beta} D_{1}^{\beta+\alpha} D_{2}^{\alpha}$ ?

Следуюший пример показывает, что даже простейшее такое соотношение с $\alpha=$ $\beta=1$ не имеет места. Рассмотрим массив

$$
a=\left(\begin{array}{ll}
1 & 1 \\
0 & 0 \\
1 & 0
\end{array}\right)
$$

Тогда

$$
\begin{aligned}
& D_{2} D_{1} D_{1} D_{2}(a)=D_{2} D_{1} D_{1}\left(\begin{array}{ll}
1 & 0 \\
0 & 1 \\
1 & 0
\end{array}\right)=D_{2}\left(\begin{array}{ll}
1 & 0 \\
0 & 1 \\
1 & 0
\end{array}\right)=\left(\begin{array}{ll}
0 & 0 \\
1 & 1 \\
1 & 0
\end{array}\right) \\
& D_{1} D_{2} D_{2} D_{1}(a)=D_{1} D_{2} D_{2}\left(\begin{array}{ll}
1 & 1 \\
0 & 0 \\
1 & 0
\end{array}\right)=D_{1}\left(\begin{array}{ll}
0 & 0 \\
1 & 1 \\
1 & 0
\end{array}\right)=\left(\begin{array}{ll}
0 & 0 \\
0 & 1 \\
2 & 0
\end{array}\right) .
\end{aligned}
$$

Забавно отметить, что эти соотношения верны как при малых $\alpha$ и $\beta$ (см. [12]), так и при больших $\alpha$ и $\beta$ (см. п. 6.7). 


\section{5. Уплотнение массивов}

5.1. ОПРеДЕЛЕНИЕ. Массив $a$ называется D-nлотнылм, если операции $D_{j}(j=$ $1,2, \ldots)$ действуют на него тривиально, т.е. $D_{j}(a)=a$ для любого $j$. Симметрично определяются $\mathbf{L}$-плотные массивы, а также $\mathbf{U}$-плотные и $\mathbf{R}$-плотные.

Вспомним (см. пп. 3.4, 3.6), как определяется действие $D_{j}$. Нужно взять стабильньй матчинг между строками $j+1$ и $j$, найти самьй правьй свободньй шар в строке $j+1$ и опустить его вниз. Тривиальность действия $D_{j}$ означает в точности, что свободных шаров в строке $j+1$ нет. То есть каждый шар в строке $j+1$ находит себе партнера (или опору) в нижележащей строке $j$.

Из леммы 2.5 мы получаем следуюший критерий $\mathbf{D}$-плотности массива.

5.2. Лемма. Массив а D-плотный тогда и только тогда, когда неравенство $a(\{1, \ldots, i\}, j+1) \leqslant a(\{1, \ldots, i-1\}, j)$ имеет место для любого $i \in I u j=$ $1, \ldots, m-1$.

В частности, если массив $a \mathbf{D}$-плотньй, то в яшиках вьше диагонали шаров нет: $a(i, j)=0$ при $j>i$. В самом деле, мы имеем последовательность неравенств

$$
a(\{1, \ldots, i\}, j) \leqslant a(\{1, \ldots, i-1\}, j-1) \leqslant \cdots \leqslant a(1, j-i+1) \leqslant a(0, j-i)=0 .
$$

На “главной” диагонали $(i=j)$ числа $a(i, i)$ расположены в невозрастающем порядке. Действительно, в силу предыдушего

$$
a(j+1, j+1)=a(\{1, \ldots, j+1\}, j+1),
$$

поэтому

$$
a(j+1, j+1)=a(\{1, \ldots, j+1\}, j+1) \leqslant a(\{1, \ldots, j\}, j)=a(j, j) .
$$

Или чуть иначе: каждый шар из ящика $(j+1, j+1)$ должен иметь "нижне-левого" партнера, а его найти можно только в ящике $(j, j)$.

5.3. ЛЕммА. Пусть массив а D-плотный. Тогда $\mathbf{D}$-плотным является любой массив $L_{i}$ а и $R_{i}$. Аналогичное утверәсдение верно для $\mathbf{L}$-плотных массивов.

В самом деле, в силу коммутирования $L_{i}$ и $D_{j}$, для любого $j$ имеем $D_{j} L_{i} a=$ $L_{i} D_{j} a=L_{i} a$.

5.4. ОПРЕДЕЛЕНИЕ. Массив $a$ назьвается LD-nлотнылм (или биплотным), если он одновременно $\mathbf{D}$-плотный и $\mathbf{L}$-плотный.

У такого массива шары лежат только в диагональных ящиках $(a(i, j)=0$, если $i \neq j)$, и при этом

$$
a(1,1) \geqslant \cdots \geqslant a(k, k) \geqslant \cdots
$$

Иными словами, биплотный массив - это просто убывающая последовательность (неотрицательных) чисел (в количестве $\min (n, m))$.

5.5. Применяя операции $D_{j}$ достаточное число раз, можно любой массив превратить в $\mathbf{D}$-плотньй. В самом деле, реальное применение операции $D_{j}$ опускает некоторьй шар нашего массива. Так как опускать ниже уровня 1 нельзя, то через конечное число таких операций все операторы $D_{j}$ будут оставлять массив на месте. 
ПРИмеР. Пусть массив $а$ имеет вид

$$
\begin{array}{|l|l|}
\hline 3 & 1 \\
\hline 1 & 1 \\
\hline 1 & 2 \\
\hline
\end{array}
$$

Стабильные матчинги соединяют шары из ящиков $(1,2)$ и $(2,3)$, а также $(1,1)$ и $(2,2)$. Шары в яшиках $(1,3)$ и $(1,2)$ не находят себе нижних партнеров. Поэтому мы можем применить $D_{1}$ или $D_{2}$. Применив $D_{1}$, мы получаем массив $D_{1} a$ вида

$$
\begin{array}{|l|l|}
\hline 3 & 1 \\
\hline 0 & 1 \\
\hline 2 & 2 \\
\hline
\end{array}
$$

Теперь можно (уже четырежды) эффективно применить $D_{2}$ и получить массив $D_{2}^{4} D_{1} a$ :

$$
\begin{array}{|l|l|}
\hline 0 & 0 \\
\hline 3 & 2 \\
\hline 2 & 2 \\
\hline
\end{array}
$$

Применяя трижды $D_{1}$, мы получаем $\mathbf{D}$-плотньй массив $D_{1}^{3} D_{2}^{4} D_{1} a$ :

$$
\begin{array}{|l|l|}
\hline 0 & 0 \\
\hline 0 & 2 \\
\hline 5 & 2 \\
\hline
\end{array}
$$

Однако можно начать с (трехкратного) применения $D_{2}$; получится массив $D_{2}^{3} a$ :

$$
\begin{array}{|l|l|}
\hline 0 & 1 \\
\hline 4 & 1 \\
\hline 1 & 2 \\
\hline
\end{array}
$$

Теперь можно четыре раза применить $D_{1}$ и получить $D_{1}^{4} D_{2}^{3} a$ :

$$
\begin{array}{|l|l|}
\hline 0 & 1 \\
\hline 0 & 1 \\
\hline 5 & 2 \\
\hline
\end{array}
$$

Наконец, подействуем $D_{2}$ и получим тот же (и это не случайно, как мы увидим дальше) $\mathbf{D}$-плотный массив $D_{2} D_{1}^{4} D_{2}^{3} a=D_{1}^{3} D_{2}^{4} D_{1} a$.

5.6. ОПРЕДЕЛЕНИЕ. $D$-словом мы называем (конечную) последовательность $\mathscr{D}=$ $D(K) \cdots D(k) \cdots D(1)$, буквы которой $D(k)$ берутся из алфавита $\left\{D_{1}, \ldots, D_{m-1}\right\}$. Аналогично понимаются $L$-слова, $U$-слова и $R$-слова.

$D$-слово $\mathscr{D}$ назьвается D-yплотняющим массив $a$, если массив $\mathscr{D} a$ является $\mathbf{D}$-плотным. Аналогично понимается $\mathbf{L}$-уплотняющее $L$-слово $\mathscr{L}$.

Например, $\mathbf{D}$-уплотняюшим является слово $\left(D_{n-1} \cdots D_{2} D_{1}\right)^{N}$ (при большом $\left.N\right)$, равно как и слово $\left(D_{1} D_{2} \cdots D_{n-1}\right)^{N}$. 
ОПРЕДЕЛЕНИЕ. $D$-слово $\mathscr{D}$ назьвается эффективны.м применительно к массиву $a$, если все массивы $a_{k}=D(k) \cdots D(1) a, k=1, \ldots, K$, различны. Аналогично понимается эффективность $L$-слов.

Очевидно, что для любого массива $a$ сушествует (причем много) эффективных D-уплотняющих слов.

Назовем формальным обращением $D$-слова $\mathscr{D}=D(K) \cdots D(1) U$-слово $\mathscr{U}=$ $U(1) \cdots U(K)$, где $U(k)=U_{j}$, если $D(k)=D_{j}$; это $U$-слово $\mathscr{U}$ можно обозначить каK $\mathscr{D}^{-1}$.

5.7. ЛЕмма. Если D-слово $\mathscr{D}$ эффективно действует на массив а, то обратное слово $\mathscr{D}^{-1}$ эффективно действует на массив $d=\mathscr{D} a, \mathscr{D}^{-1} \mathscr{D} a=а и$ $\mathscr{D} \mathscr{D}^{-1} d=d$.

5.8. Лемма. Пусть $\mathscr{D}$ - D-слово. L-слово $\mathscr{L}$ эффективно действует на массив а тогда и только тогда, когда оно әффективно действует на массив Да.

ДокАЗАТЕльство. Достаточно доказать это для $L$-слова, состоящего из одной буквы $L_{i}$. Из коммутирования имеем $L_{i} \mathscr{D} a=\mathscr{D} L_{i} a$. Если $L_{i} a=a$, то $L_{i} \mathscr{D} a=\mathscr{D} a$. Обратно, предположим, что $L_{i} a \neq a$. Тогда $i$-я столбцовая сумма массива $L_{i} a$ строго больше $i$-й столбцовой суммы массива $a$. С другой стороны, применение оператора $\mathscr{D}$ не меняет столбцовые суммы. Поэтому $\mathscr{D} L_{i} a$ отлично от $\mathscr{D} a$.

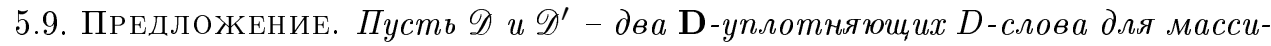
ва а. Тогда $\mathscr{D} a=\mathscr{D}^{\prime} a$.

ДокАЗАТЕльство. Рассмотрим сначала частньй случай, когда массив $a$ является L-плотным. Тогда $\mathscr{D} a$ и $\mathscr{D}^{\prime} a-$ два биплотных массива (см. лемму 5.3). Кроме того, они имеют одни и те же столбцовые суммы, так как вертикальные операции $\mathscr{D}$ и $\mathscr{D}^{\prime}$ не меняют столбцовые суммы. Значит, массивы $\mathscr{D} a$ и $\mathscr{D}^{\prime} a$ совпадают.

В общем случае пусть $\mathscr{L}-L$-слово, эффективное и $\mathbf{L}$-уплотняющее для $a$. Согласно предыдущему, $\mathscr{D}^{\prime}(\mathscr{L}(a))$ и $\mathscr{D}(\mathscr{L}(a))$ совпадают. Пусть теперь $\mathscr{R}=\mathscr{L}^{-1}$. По лемме 5.8 $\mathscr{L}$ эффективно действует на массив $\mathscr{D} a$. Поэтому $\mathscr{D} a=\mathscr{R}(\mathscr{L} \mathscr{D} a)=\mathscr{R}(\mathscr{D} \mathscr{L} a)$. Аналогично $\mathscr{D}^{\prime} a=\mathscr{R}\left(\mathscr{D}^{\prime} \mathscr{L} a\right)$. Поэтому $\mathscr{D} a=\mathscr{D}^{\prime} a$, что и доказьвает предложение.

Обозначим через $\mathbf{D} a$ массив $\mathscr{D} a$, где $\mathscr{D}$ - произвольное $\mathbf{D}$-уплотняющее массив $a$ $D$-слово. Симметрично можно говорить об операторах $\mathbf{L}, \mathbf{U}$ и $\mathbf{R}$.

5.10. СЛЕДСТВИЕ. Для любого массива а и любого D-слова $\mathscr{D}$ имеем $\mathbf{D} \mathscr{D} a=$ $\mathbf{D}$. Аналогично $\mathbf{L} \mathscr{L} a=\mathbf{L} a$ для любого L-слова $\mathscr{L}$.

В самом деле, пусть слово $\mathscr{D}^{\prime}$ уплотняющее для $\mathscr{D} a$. Тогда, очевидно, слово $\mathscr{D}^{\prime} \mathscr{D}$ уплотняет $а$. И все следует из предложения 5.9. Аналогично для $U$-слова.

5.11. Мы уже видели, что $\mathbf{L D} a=\mathrm{DL} a$ для любого массива $a$. Такой биплотный массив представляет просто убьвающую последовательность чисел (столбцовые суммы $\mathbf{L} a$ или строчные суммы $\mathbf{D} a)$. Этот массив, или убьвающая последовательность, называется формой массива $a$ и обозначается $\Phi(a)$.

5.12. ЛЕмма. $\Phi(a)=\Phi(* a)$. 
ДокАЗАтельство. Так как операции $L, R, U$ и $D$ не меняют форму, мы можем предполагать, что массив $a$ уже биплотный. Как мы знаем, в этом случае $a$ является диагональным массивом, причем количество шаров в диагональных клетках идет в убьваюшем порядке $\lambda(1) \geqslant \cdots \geqslant \lambda(k) \geqslant 0=\cdots$. Когда мы применим $*$, массив $* a$ будет располагаться на “диагонали”, идушей в клетку $(n, m)$, и шары будут расположены в обратном, возрастаюшем порядке: $\cdots=0 \leqslant \lambda(k) \leqslant \cdots \leqslant \lambda(1)$. См. второй массив на рис. 7.

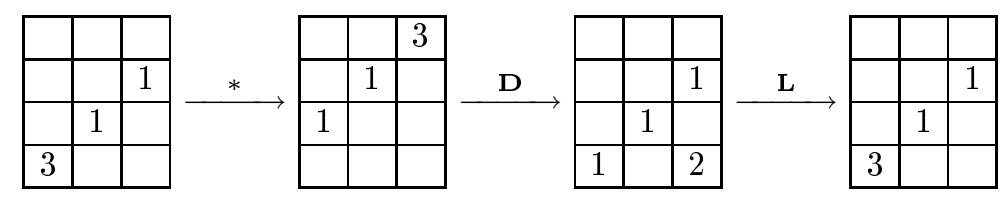

Рис. 7

Когда мы применим $\mathbf{D}$, шары расположатся линейно по диагоналям, как в третьем массиве. Наконец, когда мы применим $\mathbf{L}$, они снова соберутся на главной диагонали, причем в количестве $\lambda(1), \lambda(2), \ldots$. Так что наше утверждение проверено.

Еще одно доказательство леммы 5.12 будет дано в дополнении В.

\section{6. Теорема о биекции}

6.1. Предыдушим построениям можно придать следующую форму. Обозначим через $\mathscr{A}$ множество всех массивов (над $I \times J)$. Обозначим через $\mathbf{L} \mathscr{A}, \mathbf{D} \mathscr{A}$ и $\mathbf{B} \mathscr{A}$ множества всех L-плотных, D-плотных и биплотных массивов. Мы получаем отображение

$$
(\mathbf{L}, \mathbf{D}): \mathscr{A} \rightarrow \mathbf{L} \mathscr{A} \times \mathbf{B} \mathscr{A} \mathbf{D} \mathscr{A}
$$

множества $\mathscr{A}$ в расслоенное произведение $\mathbf{L} \mathscr{A}$ и $\mathbf{D} \mathscr{A}$ над $\mathbf{B} \mathscr{A}$ (имеются в виду естественные отображения $\mathbf{D}: \mathbf{L} \mathscr{A} \rightarrow \mathbf{B} \mathscr{A}$ и $\mathbf{L}: \mathbf{D} \mathscr{A} \rightarrow \mathbf{B} \mathscr{A})$.

6.2. Теорема. Отображсение $(\mathbf{L}, \mathbf{D}): \mathscr{A} \rightarrow \mathbf{L} \mathscr{A} \times_{\mathbf{B} \mathscr{A}} \mathbf{D} \mathscr{A}$ является биекиией.

ДОкАЗАТЕЛЬСТво. Инбективность. Пусть для массивов $a$ и $b$ выполняются равенства $\mathbf{L} a=\mathbf{L} b$ и $\mathbf{D} a=\mathbf{D} b$. Пусть $\mathscr{D}-\mathbf{D}$-уплотняющее слово для $\mathbf{L} a=\mathbf{L} b$, эффективно действующее на $\mathbf{L} a=\mathbf{L} b$. По лемме $5.8 \mathscr{D}$ эффективно действует на $a$ и на $b$. Поэтому, согласно лемме 5.7, $a=\mathscr{D}^{-1} \mathbf{D} a=\mathscr{D}^{-1} \mathbf{D} b=b$.

Сюрвективность. Пусть теперь даны $\mathbf{L}$-плотный массив $l$ и $\mathbf{D}$-плотньй массив $d$, причем $\mathbf{D} l=\mathbf{L} d$. И пусть $\mathscr{D}-\mathbf{D}$-уплотняюшее слово для $l$, эффективно действуюшее на $l$. Тогда по лемме $5.7 l=\mathscr{D}^{-1} \mathbf{D} l$, и $U$-слово $\mathscr{D}^{-1}$ эффективно действует на $\mathbf{D} l=$ $\mathbf{L} d$. По лемме $5.8 \mathscr{D}^{-1}$ эффективно действует на $d$, и поэтому $\mathscr{D} \mathscr{D}^{-1} d=d$. Положим $a=\mathscr{D}^{-1} d$. Тогда $\mathbf{L} a=\mathbf{L} \mathscr{D}^{-1} d=\mathscr{D}^{-1} \mathbf{L} d=\mathscr{D}^{-1} \mathbf{D} l=l$. Так как $\mathscr{D}$ уплотняющее и эффективное $D$-слово для $l=\mathbf{L} a$, то оно уплотняюшее и эффективное слово для $a$. По лемме $5.7 \mathbf{D} a=\mathscr{D} a=\mathscr{D}^{-1} d=d$. Теорема 6.2 доказана.

6.3. Отображение $(\mathbf{L}, \mathbf{D})$ согласовано с операциями $L, D, R, U$, если их правильно определить на расслоенном произведении. Пусть дана "согласованная" пара массивов $(l, d)$, где $l \mathbf{L}$-плотный, $d \mathbf{D}$-плотньй массивы, и $\mathbf{D} l=\mathbf{L} d$. Положим

$$
L_{i}(l, d)=\left(L_{i} l, L_{i} d\right)=\left(l, L_{i} d\right)
$$


По лемме 5.3 массив $L_{i} d$ снова $\mathbf{D}$-плотный. Кроме того, $\mathbf{L} L_{i} d=\mathbf{L} d=\mathbf{D} l$ в силу следствия 5.10. Поэтому это действие $L_{i}$ определено корректно. Аналогично определяются действия $R_{i}, D_{j}$ и $U_{j}$.

При таком определении действий они коммутируют с отображением $(\mathbf{L}, \mathbf{D})$. B caмом деле, если $l=\mathbf{L} a$, а $d=\mathbf{D} a$, то $\mathbf{L} L_{i} a=\mathbf{L} a=l$, а $\mathbf{D} L_{i} a=L_{i} \mathbf{D} a=L_{i} d$. То есть $(\mathbf{L}, \mathbf{D})$ коммутирует с $L_{i}$. Аналогично оно коммутирует с операторами $R, D$ и $U$.

6.4. Уплотняющие слова. При построении биекции в теореме 6.2 важную роль играли $D$-слова, эффективно $\mathbf{D}$-уплотняющие $\mathbf{L}$-плотный массив $l$. Предложим один конкретный способ построения таких слов.

1) Начинаем с этажей 1 и 2 . На первом этаже шары есть только в ящике $(1,1)$, на втором - в ящиках $(1,2)$ и $(2,2)$. Причем в силу $\mathbf{L}$-плотности массива $l$ все шары из ящика $(2,2)$ имеют (левых) партнеров в яшике $(1,1)$. Поэтому в стабильном матчинге между первой и второй строчками все шары в ящике $(2,2)$ находят (нижних) партнеров. Шары в яшике $(1,2)$ партнеров, естественно, не находят. Поэтому на втором этаже есть ровно $l(1,2)$ свободных шаров. Это означает, что слово $D_{1}^{l(1,2)}$ эффективно действует на $l$ и перемешает все шары из яшика $(1,2)$ в яшик $(1,1)$.

2 ) Теперь мы имеем новьй массив $l_{1}=D_{1}^{l(1,2)} l$. У него яшик $(1,2)$ пустой. Так как $l_{1}$ снова $\mathbf{L}$-плотный массив, все шары из яшика $(3,3)$ имеют партнеров в ящике $(2,2)$. C другой стороны, все шары в яшиках $(1,3)$ и $(2,3)$ не имеют "нижней опоры”. Поэтому слово $D_{2}^{l(1,3)+l(2,3)}$ эффективно действует на $l_{1}$, очищая ящики $(1,3)$ и $(2,3)$. При этом в яшике $(1,2)$ появляются шары в количестве $l(1,3)$. Если теперь мы подействуем (эффективно) словом $D_{1}^{l(1,3)}$, то получим массив $l_{2}$ с пустыми яшиками $(1,2),(1,3)$ и $(2,3)$. Вьше третьего этажа массив $l_{2}$ совпадает с исходным $l$.

3 ) Теперь начинаем применять $D_{3}$ и действуем этим оператором $l(1 . .3,4)=l(1,4)+$ $l(2,4)+l(3,4)$ раз. Яшики $(1,4),(2,4)$ и $(3,4)$ освобождаются, но наполняются яшики $(1,3)$ и $(2,3)$. Действуем теперь словом $D_{2}^{l(1 . .2,4)}$. Наконец, вычищаем ящик $(1,2)$, применяя $D_{1}^{l(1,4)}$. Получаем массив $l_{3}$.

4) Применяя $D_{4}$ нужное число раз, затем $D_{3}, D_{2}$ и $D_{1}$, получая массив $l_{4}$. И так далее. Обшая формула для очередного массива $l_{j}$ вьглядит так:

$$
l_{j}=D_{1}^{l(1 . .1, j+1)} \cdots D_{j-1}^{l(1 . . j-1, j+1)} D_{j}^{l(1 . . j, j+1)} l_{j-1} .
$$

6.5. Если мы не хотим следить за точньм количеством итераций каждой операции $D_{j}$ (и тем самым за эффективностью действия), можно ввести обозначение $\mathbf{D}_{j}=$ $D_{j}^{\infty}$. Тогда переход от $l_{j-1} \mathrm{k} l_{j}$ выглядит компактнее:

$$
l_{j}=\mathbf{D}_{1} \cdots \mathbf{D}_{j} l_{j-1} .
$$

Теперь можно написать формулу для окончательного биплотного массива $\mathbf{D} l=l_{m-1}$

$$
\mathbf{D} l=\left(\mathbf{D}_{1} \cdots \mathbf{D}_{m-1}\right)\left(\mathbf{D}_{1} \cdots \mathbf{D}_{m-2}\right) \cdots\left(\mathbf{D}_{1} \mathbf{D}_{2}\right) \mathbf{D}_{1} l .
$$

Заметим, что эта формула дает $\mathbf{D}$-уплотнение произвольного массива $a$ :

$$
\mathbf{D} a=\left(\mathbf{D}_{1} \cdots \mathbf{D}_{m-1}\right)\left(\mathbf{D}_{1} \cdots \mathbf{D}_{m-2}\right) \cdots\left(\mathbf{D}_{1} \mathbf{D}_{2}\right) \mathbf{D}_{1} a .
$$


6.6. Впрочем, это не единственньй способ написать $\mathbf{D}$-уплотнение. Можно сначала подействовать $\mathbf{D}_{1}$, "вычистив" вторую строку. Затем применить $\mathbf{D}_{2}$, вычишая третью строку, но засоряя вторую. Затем $\mathbf{D}_{3}$ и т. д. В результате мы вычишаем самую верхнюю ( $m$-ю) строку. Теперь все повторяем: $\mathbf{D}_{1}, \mathbf{D}_{2}$ и т. д., очишая строку $(m-1)$. И так далее. Получается другая формула для $\mathbf{D}$-уплотнения:

$$
\mathbf{D} a=\mathbf{D}_{1}\left(\mathbf{D}_{2} \mathbf{D}_{1}\right) \cdots\left(\mathbf{D}_{m-1} \mathbf{D}_{m-2} \cdots \mathbf{D}_{1}\right) a \text {. }
$$

Имеется много других способов D-уплотнения "обшего" массива. Все они получаются один из другого с помошњю следуюших трех основных соотношений между операторами $\mathbf{D}_{j}$ (вырожденная алгебра Гекке $\left.H_{m}(0)\right)$.

6.7. ПРЕДЛОЖЕНИЕ. а) $\mathbf{D}_{j} \mathbf{D}_{j}=\mathbf{D}_{j}$.

b) $\mathbf{D}_{j}$ и $\mathbf{D}_{k}$ коммутируют, если $j$ и $k$ несоседние индексь.

c) $\mathbf{D}_{j} \mathbf{D}_{j+1} \mathbf{D}_{j}=\mathbf{D}_{j+1} \mathbf{D}_{j} \mathbf{D}_{j+1}$ для любого $j$.

ДокАЗАТЕльство. Соотношение а) очевидно из определения $\mathbf{D}_{j}$. b) следует из коммутирования $D_{j}$ и $D_{k}$ для несоседних $j$ и $k$ (см. п. 4.6). Остается доказать с). Без ограничения общности мы можем считать $j=1$. А также, что наш массив $a$ является L-плотным. Мы уже видели, что левое слово D-уплотняет массив. Остается проверить, что это же делает правое слово. Применение $\mathbf{D}_{2}$ “очищает” яшик $(1,3)$. Применение $\mathbf{D}_{1}$ очищает ящик $(1,2)$; жик $(1,3)$ при этом как был пустьм, так и остается пустым. Наконец, применение $\mathbf{D}_{2}$ очищает и ящик $(2,3)$, и мы получаем “диагональньй”, т.е. биплотньй, массив.

Кстати, применение этого принципа дает еще одну формулу:

$$
\mathbf{D} a=\mathbf{D}_{m-1}\left(\mathbf{D}_{m-2} \mathbf{D}_{m-1}\right) \cdots\left(\mathbf{D}_{2} \cdots \mathbf{D}_{m-1}\right)\left(\mathbf{D}_{1} \cdots \mathbf{D}_{m-1}\right) a .
$$

6.8. Приведенные разложсения. Пункты 6.8-6.10 не нужны для понимания дальнейшего и призваны лишь указать связи с другими темами.

Соотношения из предложения 6.7 выглядят почти как соотношения Мура-Кокстера (см. п. А.1) для симметрической групш; разница только в соотношении а). Польза этого замечания такая. Обозначим, как это принято, через $w_{0}$ "обратную" перестановку $(m, m-1, \ldots, 2,1)$ в симметрической групе $S_{m}=S(J)$. Как и любая перестановка, $w_{0}$ может быть записана как произведение инволюций $\sigma_{1}, \ldots, \sigma_{m-1}\left(\sigma_{j}\right.$ переставляет соседние $j$ и $j+1)$. Например, $w_{0}=\left(\sigma_{1} \cdots \sigma_{m-1}\right) \cdots\left(\sigma_{1} \sigma_{2}\right) \sigma_{1}$. Это представление имеет минимальную длину $N=m(m-1) / 2$. Есть много других представлений такой же минимальной длины; множество их обозначим $R=R\left(w_{0}\right)$. (Кстати, сушествует нетривиальная биекция Грина-Эдельмана между $R$ и множеством стандартных таблиц формы $(m-1, m-2, \ldots, 2,1)$.) Любой элемент из $R$ назовем легендой (А.В. Зелевинский назьвает их направлениями); это некоторая (не любая) последовательность $N$ индексов $\left(i_{N}, \ldots, i_{1}\right), i_{s}=1, \ldots, m-1$.

Назовем легенды соседними, если они отличаются либо двумя соседними буквами $(\ldots i j \ldots$ и $\ldots j i \ldots$, причем $|i-j|>1)$, либо тремя соседними буквами $(\ldots i j i \ldots$ и $\ldots j i j \ldots$, и $|i-j|=1)$. Известно, что любые две легенды можно соединить цепочкой соседних.

Из этого следует, что если $\mathbf{i}=\left(i_{N}, \ldots, i_{1}\right)$ - легенда, то оператор $\mathbf{D}_{i_{N}} \cdots \mathbf{D}_{i_{1}}$ задает $\mathbf{D}$-уплотнение, т.е. совпадает с $\mathbf{D}$. 
6.9. Параметризачии канонического базиса. Выше, в пш. 6.5-6.7, мы не следили за степенями $D_{j}$, необходимьми для уплотнения массива. Оказьвается полезно проследить и за степенями.

Пусть $z=(z(i, j))$ - массив из чисел, стоящих вьше диагонали (т.е. $z(i, j)=0$ при $j \leqslant i \leqslant m)$. Иными словами, такой массив - это точка в ортанте $\mathbb{R}_{+}^{N}$ (где $N=$ $m(m-1) / 2$, как и в п. 6.7). Поместим в диагональных клетках достаточно быстро убьваюшие числа (которые не будут в дальнейшем никак участвовать) так, чтобы наш массив был $\mathbf{L}$-плотньм.

Выберем теперь некоторую легенду $\mathbf{i}=\left(i_{N}, \ldots, i_{1}\right)$, как в п. 6.7, и будем осушествлять $\mathbf{D}$-уплотнение в соответствии со следующим простым рецептом. Действуем оператором $D_{i_{1}}$ на $z$ до тех пор, пока он действует эффективно. Более точно, мы действуем им в степени $\delta_{i_{1}}(z)$ ( $\delta$ здесь - это дефект из п. 3.3$)$. В результате получаем массив $z^{\prime}=D_{i_{1}}^{\delta_{i_{1}}(z)}(z)$. С этим массивом мы повторяем предыдущую инструкцию, но используя теперь оператор $D_{i_{2}}$. И так далее до $i_{N}$. Грубо говоря, мы идем по направлению $i_{1}$ до тех пор, пока не упремся; длину пройденного на этом первом этапе пути, т.е. $\delta_{i_{1}}(z)$, обозначим как $t_{1}$. Затем идем в направлении $i_{2}$ до упора и пройденный путь отмечаем как $t_{2}$. И так далее. В результате мы получаем набор $\left(t_{N}, \ldots, t_{1}\right)$ неотрищательных чисел ("струну" $z$ в направлении $\mathbf{i}$ ), который обозначается $c_{\mathbf{i}}(z)$. Тем самым мы получаем отображение $c_{\mathbf{i}}$ из $\mathbb{R}_{+}^{N}$ в $\mathbb{R}_{+}^{N}$.

Мы утверждаем, что отображение $c_{\mathbf{i}}$ является вложением. Это следует из двух замечаний. Во-первых, применение слова $D_{i_{N}}^{t_{N}} \cdots D_{i_{1}}^{t_{1}}$ к $z$ производит $\mathbf{D}$-уплотнение массива $z$. С другой стороны, в силу эффективности действия $D$-слова $D_{i_{N}}^{t_{N}} \cdots D_{i_{1}}^{t_{1}}$ на массив $z$ обратное слово восстанавливает $z$ из $\mathbf{D} z$.

Можно показать, что отображение $c_{\mathbf{i}}$ кусочно линейное и что его образ $C_{\mathbf{i}}$ является вьпукльп (полиэдральным) конусом. Кроме того, это отображение, очевидно, переводит целые массивы в целые струны, т.е. задает биекцию $\mathbb{Z}_{+}^{N}$ на $C_{\mathbf{i}}(\mathbb{Z})$. Реальное значение этих биекций в том, что они дают различные параметризации канонического базиса (что это такое и зачем нужно, читатель может узнать из статей А. В. Зелевинского и А. Д. Бернштейна [5], [6], [53]; см. также [24]). Кроме того, для соседних легенд довольно легко переходить от одной параметризации к другой. Рассмотрим более подробно простейший случай $m=3$. (В силу теоремы коммутирования 4.2 этим случаем можно и ограничиться.)

6.10. ПримеР. Тут у нас есть только две легенды: 121 и 212. Вычислим соответствующие струны для массива $z$. Выгисление для легенды 121 уже было проделано в п. 6.5 и дает такой ответ:

$$
c_{121}(z)=(z(1,3), z(1,3)+z(2,3), z(1,2)) .
$$

Действуя согласно легенде 212 , мы сначала должны применить $\mathbf{D}_{2}$ к массиву $z$ вида

\begin{tabular}{|c|c|}
\hline$z(1,3)$ & $z(2,3)$ \\
\hline$z(1,2)$ & 0 \\
\hline$\infty$ & 0 \\
\hline
\end{tabular}

Какой путь $t_{1}$ мы при этом пройдем? То есть сколько шаров мы опустим с третьего этажа? В яшике $(2,3)$ поддержку имеют $\min (z(1,2), z(2,3))$ шаров; остальные опускаются. Кроме того, опускаются все шары из ящика $(1,3)$. Поэтому $t_{1}=z(1,3)+$ 
$\max (z(2,3)-z(1,2), 0)$. Кроме того, в результате применения $D_{2}^{t_{1}}$ мы получим массив $z^{\prime}$

\begin{tabular}{|c|c|}
\hline 0 & $\min (z(1,2), z(2,3))$ \\
\hline$z(1,2)+z(1,3)$ & $t_{1}$ \\
\hline$\infty$ & 0 \\
\hline
\end{tabular}

Теперь уже ясно, что нужно применить $t_{2}=z(1,2)+z(1,3)$ раз операцию $D_{1}$ и затем $t_{3}=\min (z(1,2), z(2,3))$ раз операцию $D_{2}$. Так что струна $c_{212}(z)$ имеет вид

$$
c_{212}(z)=(\min (z(2,3), z(1,2)), z(1,2)+z(1,3), z(1,3)+\max (z(2,3)-z(1,2), 0)) .
$$

Переход от первой параметризации ко второй, т.е. от конуса $C_{121}$ к конусу $C_{212}$ (совпадающему в данном случае с $C_{121}$ ), задается формулами

$$
z^{\prime}=\min (x+z, y)-z, \quad y^{\prime}=x+z, \quad x^{\prime}=\max (x+z, y)-x .
$$

\section{7. Массивы как кристаллы}

7.1. Связь с кристаллами. Наличие, скажем, вертикальных операций $D_{j}$ и $U_{j}$ позволяет говорить о (целочисленных) массивах как о кристаллах. Нам не хотелось бы утяжелять рассказ отвлечением про кристаллы (подробнее об этом см. [31], [30], [12], [37]). Скажем только, что понятие кристалла определено для любой системы корней (и выделенного базиса простых корней); в этом смысле массивы образуют кристалл для системы $\mathbb{A}_{m-1}$ (или, лучше, для системы $\mathbb{A}(J)$ и корней вида $e_{j+1}-e_{j}$, $j=1, \ldots, m-1)$. Такой кристаллический взгляд на вещи часто помогал нам находить правильные понятия и определения.

Отметим две особенности нашей "массивной" ситуации. Во-первых, кроме вертикальных операций $D$ и $U$ у нас имеются горизонтальные операции $L$ и $R$. Это дает другую кристаллическую структуру (для системы корней $\mathbb{A}(I))$ на $\mathscr{A}$, коммутируюшую с "вертикальной" кристаллической структурой. Можно сказать, что $\mathscr{A}$ является бикристаллом. Во-вторых, конструкция переносится на вешественные массивы (и дробные степени $D$ и $U$ ) и приводит к понятию "непрерьвного" кристалла, как бы дико это ни звучало.

7.2. Будем рассматривать для определенности действие вертикальных операций $D$ и $U$. Попросту говоря, мы имеем действие полугруппы, свободно порожденной этими операторами. Замечательная особенность ситуации в том, что операторы $D$ и $U$ "почти" обратны друг к другу. Так что хотя наша полугруппа формально и не является групой, она "почти" группа. Во всяком случае мы можем говорить об инвариантных подмножествах массивов и об орбитах. Уточним сказанное, ориентируясь на целочисленные массивы и целые степени операторов $D$ и $U$ (чтобы быть максимально близко к кристаллам).

ОПРЕДЕЛЕНИЕ. Подмножество $A$ в $\mathscr{A}_{\mathbb{Z}}(I \times J)$ назьвается инвариантныц (или $D$ - $U$-инвариантным), если оно инвариантно относительно применения любых операторов $D_{j}$ и $U_{j}$.

Например, фиксируем некоторый вес $x \in \mathbb{Z}_{+}^{I}$. Тогда множество $S(x)$ всех массивов $I$-веса $x$ является инвариантным подмножеством. В самом деле, операции $D_{j}$ и $U_{j}$ сохраняют $I$-вес. 
Очевидно, что пересечение или объединение инвариантных подмножеств снова инвариантно. Почти столь же очевидно, что и дополнение тоже инвариантно. В самом деле, пусть мы имеем два инвариантных подмножества $A^{\prime} \subset A$; проверим, что $A \backslash A^{\prime}$ тоже инвариантно. Представим, что массив $a \in A \backslash A^{\prime}$ под действием, скажем, оператора $D_{j}$ вьшел из $A \backslash A^{\prime}$, т.е. попал в $A^{\prime}$. Но это значит, что $D_{j} a \neq a$. А тогда по предложению $3.5 a=U_{j} D_{j} a$ и в силу инвариантности $A^{\prime}$ массив $a$ тоже принадлежит $A^{\prime}$. Противоречие.

Минимальное (непустое) инвариантное подмножество назьвается орбитой. Иначе говоря, из любого массива орбиты в любой другой можно перейти с помощью серии операций $D_{j}$ и $U_{j}$. (В каком-то смысле инвариантное подмножество - это аналог представления группы $G L(m)$, а орбита - аналог неприводимого представления.) Из предыдущего утверждения мы получаем следующий аналог полной приводимости.

7.3. ЛЕмМА. Любое инвариантное множество есть непересекающееся обтединение орбит.

Легко понять, как устроена произвольная орбита $O$. Пусть $a \in O$. Тогда массив $\mathbf{D} a$ также принадлежит $O$. Более того, он не зависит от выбора массива $a$ внутри $O$ (см. п. 5.10). Поэтому его можно обозначить $\mathbf{D} O$ и назвать нижним концом орбиты $O$. Ясно, что нижний конец однозначно определяет орбиту. Обратно, если взять произвольный $D$-плотньй массив $d$, то он будет нижним концом своей орбиты. Тем самым мы получаем следующее предложение.

7.4. ПРЕДЛОЖЕНИЕ. Орбиты находятся в биективном соответствии с Dплотными массивами. Для D-плотного массива d его орбита $O(d)$ состоит из массивов вида $\mathscr{U} d$, где $\mathscr{U}$ пробегает множество $U$-слов.

7.5. ПРимеР. Зафиксируем разбиение $\mu=(\mu(1), \ldots, \mu(n))$ и обозначим той же буквой биплотньй массив формы $\mu$. Обозначим через $\mathscr{A}_{\mathbb{Z}}(* \backslash \mu)$ множество массивов $a$ таких, что “двухэтажный” массив $\left(\begin{array}{l}a \\ \mu\end{array}\right)(a$ стоит над $\mu$ ) является L-плотным. По теореме о коммутировании это множество $\mathscr{A}_{\mathbb{Z}}(* \backslash \mu)$ инвариантно. Орбиты этого множества состоят из таких $\mathbf{D}$-плотных массивов $d$, что $\left(\begin{array}{l}d \\ \mu\end{array}\right)$ является $\mathbf{L}$-плотньм массивом.

Обычно тут еще фиксируют $I$-вес (или форму) $\lambda$ L-плотного массива $\left(\begin{array}{l}a \\ \mu\end{array}\right)$. Конечно, это означает в точности, что $I$-вес массива $a$ равен $\lambda-\mu$. Получившееся инвариантное множество обозначим $\mathscr{A}_{\mathbb{Z}}(\lambda \backslash \mu)$. Орбиты параметризуются $\mathbf{D}$-плотньми массивами $d$ $I$-веса $\lambda-\mu$ такими, что “двухэтажньй” массив $\left(\begin{array}{l}d \\ \mu\end{array}\right) \mathbf{L}$-плотньй.

7.6. Итак, задать орбиту - значит задать $\mathbf{D}$-плотньй массив $d$ (или, что то же самое, полустандартную таблицу, см. предложение 9.3 ниже). Все массивы орбиты $O(d)$ имеют один и тот же $I$-вес, совпадающий, конечно, с $I$-весом массива $d$.

Все массивы из орбиты $O(d)$ имеют также одну и ту же форму, совпадающую с формой $d$, т.е. с $J$-весом $d$. Если обозначить через $\mathscr{L}$ произвольное $L$-уплотняющее слово, то $\mathscr{L}$ задает отображение орбиты $O(d)$ в орбиту $O(\mathbf{L} d)$. Это отображение

$$
\mathscr{L}: O(d) \rightarrow O(\mathbf{L} d)
$$

во-первых, является биекцией множеств, а во-вторых, согласовано с действиями $D$ и $U$. Иначе говоря, эти орбиты изоморфны как кристаллы.

Биплотньй массив $\mathbf{L} d$ можно отождествить с разбиением $\lambda$ (см. п. 5.4). Орбиту $O(\lambda)$ этого биплотного массива $\lambda$ будем назьвать эталонной орбитой формы $\lambda$. Она состоит из L-плотных массивов формы $\lambda$. Любая орбита изоморфна эталонной. 
Таким образом, задать орбиту с точностью до изоморфизма - значит задать некоторое разбиение $\lambda$ с $m$ частями (будем считать для простоты, что $n \geqslant m$ ). Число элементов в орбите равно размерности соответствующего неприводимого представления $V_{\lambda}$ (см. п. 1.5). Это лишний раз подчеркивает аналогию с представлениями $G L(m)$. Преимушество же в том, что даже изоморфные "представления"-орбиты четко различаются по своим нижним концам (аналогам векторов старшего веса).

7.7. ПримеРы. а) Пусть $\lambda=(1,0, \ldots, 0)=(1)$. Орбита $O(1)$ состоит из $m$ массивов; каждый массив содержит один шар в первом столбце. Это аналог стандартного представления $G L(m)$.

b) Пусть $\lambda=(k, 0, \ldots, 0)=(k)$, где $k$-целое неотрицательное. Орбита $O(k)$ образована всеми (целочисленными) массивами, у которых $k$ шаров размешаются в первом столбце (а остальные столбцы пустые). Это аналог $k$-й симметрической степени стандартного представления.

с) Пусть $\lambda=(1, \ldots, 1,0, \ldots, 0)=\left(1^{k}\right)$. Как устроена орбита $O\left(1^{k}\right)$ ? Каждый массив из $O\left(1^{k}\right)$ содержит один шар в каждом из первых $k$ столбцов. При этом, как легко понять, каждый следуюший шар должен располагаться строго вьше предыдушего. Так что задать массив из $O\left(1^{k}\right)$ - значит задать строго монотонное отображение из $\{1, \ldots, k\}$ в $J=\{1, \ldots, m\}$. Или, что то же самое, задать $k$-элементное подмножество в $J$. Отсюда видно, что $O\left(1^{k}\right)$ является аналогом $k$-й внешней степени стандартного представления.

d) Пусть $m=3$ и $\lambda=(2,1)$. Орбита $O(\lambda)$ состоит из 8 массивов:

$$
\begin{aligned}
& a=\begin{array}{l|l|}
\hline 0 & 0 \\
\hline 0 & 1 \\
\hline 2 & 0 \\
\hline 1 & 0 \\
\hline 0 & 1 \\
\hline 1 & 0 \\
\hline
\end{array}, \quad b=\begin{array}{|l|l|}
\hline 0 & 1 \\
\hline 0 & 0 \\
\hline 2 & 0 \\
\hline 0
\end{array}, \quad c=\begin{array}{|l|l|l|}
\hline 0 & 0 \\
\hline 1 & 1 \\
\hline 1 & 0 \\
\hline 2 & 0 \\
\hline 0 & 0 \\
\hline
\end{array}, \quad g=\begin{array}{|l|l|}
\hline 1 & 1 \\
\hline 1 & 0 \\
\hline 0 & 0 \\
\hline
\end{array}, \quad h=\begin{array}{|l|l|}
\hline 0 & 1 \\
\hline 1 & 0 \\
\hline 1 & 0 \\
\hline 1 & 1 \\
\hline 0 & 0 \\
\hline 1 & 0 \\
\hline
\end{array} .
\end{aligned}
$$

Наглядно (как кристалл Кашивары над решеткой $\mathbb{Z} \otimes J=\mathbb{Z}^{3}$ ) эта орбита $O_{3}(2,1)$ выглядит как на рис. 8.

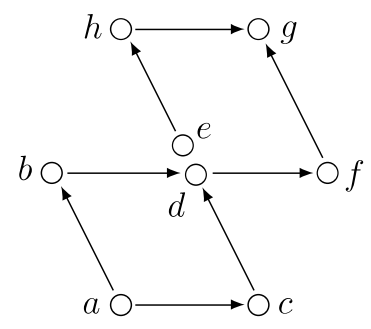

Рис. 8. Горизонтальные стрелки обозначают действие оператора $U_{1}$, а наклонные - оператора $U_{2}$

7.8. Морфизмы. Пусть $A$ и $A^{\prime}$ - два инвариантных множества целочисленных массивов (быть может, с разными $I$ и $I^{\prime}$, но одним и тем же $J$ ). Морфизмом $A$ в $A^{\prime}$ назовем отображение $f: A \rightarrow A^{\prime}$, коммутирующее с действиями $D_{j}$ и $U_{j}, j=1, \ldots, m-1$. 
Например, пусть $\mathscr{R}$ - некоторое $R$-слово. Тогда сопоставление $a \mapsto \mathscr{R} a$ задает морфизм $\mathscr{A}_{\mathbb{Z}}$ в себя (теорема 4.2 ). Аналогично для $L$-слов.

Пусть $O$ и $O^{\prime}-$ две орбиты с нижними концами $d$ и $d^{\prime}$, и пусть $f-$ морфизм $O$ в $O^{\prime}$. Тогда очевидно, что $f(d)=d^{\prime}$ (и аналогично для верхних конщов). Если $a \in O$ имеет вид $a=\mathscr{U} d$ (где $\mathscr{U}$ - некоторое $U$-слово), то $f(a)=f(\mathscr{U} d)=\mathscr{U} f(d)=\mathscr{U} d^{\prime}$. Поэтому морфизм $f$ однозначно определен и сюръективен.

Если $d^{\prime}=\mathscr{R} d$ для некоторого $R$-слова $\mathscr{R}$, то соответствуюший морфизм $\mathscr{R}: O(d) \rightarrow$ $O\left(d^{\prime}\right)$ является изоморфизмом. Не всегда морфизм между орбитами является изоморфизмом. Например, если орбита $O^{\prime}$ состоит из единственного массива $d^{\prime}$ (которьй одновременно $\mathbf{D}$-плотньй и $\mathbf{U}$-плотньй), тогда единственное отображение любой орбиты $O$ в $O^{\prime}=\left\{d^{\prime}\right\}$ является морфизмом. Однако это единственное исключение. И имеет место следуюший аналог леммы Шура.

7.9. ТеоремА. Пусть $f: O \rightarrow O^{\prime}-$ морфизм двух орбит. Тогда либо $f$ биективен, либо орбита $O^{\prime}$ состоит из единственного массива.

ДокАЗАТЕльство. Пусть $f: O \rightarrow O^{\prime}$ морфизм орбит. Мы уже говорили, что $f$ сюръективен. Предположим, что $f$ не инъективен, т.е. $f(a)=f\left(a^{\prime}\right)$ для двух различных массивов из орбиты $O$. Назовем такую пару массивов двойняшками. Если $a$ и $a^{\prime}$ двойняшки, тогда либо $D_{j} a$ и $D_{j} a^{\prime}$ различны (и тоже двойняшки), либо $D_{j} a=D_{j} a^{\prime}$.

Действуя операторами $D_{j}$, найдем самую “нижнюю” пару двойняшек $a$ и $a^{\prime}$. Это значит, что (для любого $j$ ) $D_{j} a=D_{j} a^{\prime}$. Ясно, что $a$ и $a^{\prime}$ не могут одновременно быть неподвижными относительно действия $D_{j}$. Не могут они и одновременно двигаться, так как в этом случае $a=U_{j} D_{j} a=U_{j} D_{j} a^{\prime}=a^{\prime}$. Значит, один из них, скажем, $a$ неподвижен при действии $D_{j}$, а другой $-a^{\prime}-$ двигается, $D_{j} a^{\prime} \neq a^{\prime}$.

Но тогда $f(a)=f\left(D_{j} a\right)=D_{j} f(a)$, т.е. $f(a)$ - плотньй вниз. Но $f(a)$ одновременно и плотный вверх! В самом деле, $U_{j} f(a)=U_{j} f\left(D_{j} a\right)=U_{j} f\left(D_{j} a^{\prime}\right)=f\left(U_{j} D_{j} a^{\prime}\right)=$ $f\left(a^{\prime}\right)=f(a)$.

7.10. ЗАмЕчАНИЕ. Легко понять, что эталонные орбиты $O(\lambda)$ и $O(\mu)$ изоморфин тогда и только тогда, когда разбиения $\lambda$ и $\mu$ отличаются на константу. Можно сказать, что переход к кристаллическим графам склеивает неприводимые представления, отличающиеся подкруткой на степень определителя, т.е. на $O\left(1^{m}\right)$.

7.11. Изоморфизм Шютиенберже. Важньй пример изоморфизма дает конструкция, навеянная понятием инволюции Шютценберже. Для этого вспомним про операцию $*$ центральной симметрии массива из п. 2.3 .

Пусть $A$ - инвариантное множество массивов формата $I \times J$. Рассмотрим множество $* A=\{* a, a \in A\}$. Очевидно, что оно тоже инвариантное (см. п. 3.6). И хотя само отображение $*$ не является морфизмом, мы утверждаем, что существует канонический изоморфизм $S c h=S c h_{I}: A \rightarrow * A$. Он однозначно определен тем, что переводит орбиту массива $a \in A$ в орбиту массива $* a$. Так как, согласно лемме 5.12 , форма массива $а$ совпадает с формой массива $* a$, орбиты $O=O(a)$ и $* O=O(* a)$ канонически изоморфны.

Если $d$ - нижний конец орбиты $O$, то нижний конец орбиты $* O$ равен $\mathbf{D}(* d)$. Покажем, как это вьглядит на конкретном примере. Пусть $\mathbf{D}$-плотный массив $d$ имеет вид

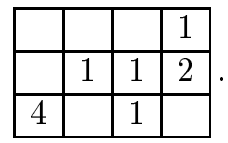


Перевернутьй массив $* d$ имеет вид

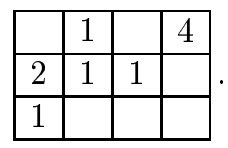

Делая уплотнение вниз, мы получаем $S \operatorname{ch}(d)$ :

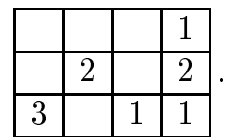

Обратим внимание, что исходньй $I$-вес $(4,1,2,3)$ поменялся на противоположный $(3,2,1,4)$.

\section{8. Тензорное произведение массивов}

8.1. Конкатеначия массивов. До сих пор мы работали с массивами фиксированного формата $I \times J$. Теперь мы, оставив фиксированньм вертикальньй алфавит $J$, будем варьировать горизонтальные алфавиты. Пусть имеются два алфавита, $I$ и $I^{\prime}$. Через $I \sqcup I^{\prime}$ обозначим (непересекающееся) объединение $I$ и $I^{\prime}$, сохраняя старые порядки в $I$ и $I^{\prime}$ и считая элементы из $I^{\prime}$ больше элементов $I$.

Пусть теперь $a$ - массив формата $I \times J$, а $a^{\prime}-$ массив формата $I^{\prime} \times J$. Помешая массив $a^{\prime}$ после $a$, мы получаем массив $\left(a, a^{\prime}\right)$ формата $\left(I \sqcup I^{\prime}, J\right)$. Естественно задаться вопросом: как действует операция $D_{j}$ на такой составной массив? Здесь можно считать, что массивы $a$ и $a^{\prime}$ имеют только две строчки (и обозначать $D_{j}$ просто как $D$ ).

Обозначим через $\mu$ стабильньй матчинг для $a$, а через $\mu^{\prime}-$ для $a^{\prime}$. В массиве $a$ имеется $\varepsilon(a)$ свободных нижних шаров (мы тут обрашаемся к определениям из пп. $3.2,3.3$ ), а массив $a^{\prime}$ имеет $\delta\left(a^{\prime}\right)$ свободных верхних шара. Чтобы получить матчинг для составного массива $\left(a, a^{\prime}\right)$, мы должны спарить эти свободные шары между собой. Возможны две ситуации.

Первая: $\varepsilon(a) \geqslant \delta\left(a^{\prime}\right)$. Тогда все свободные верхние шары в массиве $a^{\prime}$ получают себе партнеров. И в массиве $\left(a, a^{\prime}\right)$ свободные верхние шары - это в точности свободные верхние шары массива $a$. Отсюда видно, что

$$
D\left(a, a^{\prime}\right)=\left(D a, a^{\prime}\right)
$$

и что $\delta\left(a, a^{\prime}\right)=\delta(a), \varepsilon\left(a, a^{\prime}\right)=\varepsilon\left(a^{\prime}\right)+\varepsilon(a)-\delta\left(a^{\prime}\right)$.

Вторая: $\varepsilon(a)<\delta\left(a^{\prime}\right)$. Теперь только $\varepsilon(a)$ свободных верхних шаров найдут себе свободных партнеров. Причем те, которые стоят левее. А остальные $\delta\left(a^{\prime}\right)-\varepsilon(a)$ так и останутся свободными. Теперь уже ясно, что в этом случае

$$
D\left(a, a^{\prime}\right)=\left(a, D a^{\prime}\right),
$$

$\delta\left(a, a^{\prime}\right)=\delta(a)+\delta\left(a^{\prime}\right)-\varepsilon(a), \varepsilon\left(a, a^{\prime}\right)=\varepsilon\left(a^{\prime}\right)$.

В каком-то смысле эти формулы можно рассматривать как определение операции $D$.

8.2. Тензорное произведение. Пусть теперь $A$-инвариантное (относительно операций $D$ и $U)$ подмножество $(I \times J)$-массивов, а $A^{\prime}$ - инвариантное подмножество $\left(I^{\prime} \times J\right)$-массивов. Назовем тензорным произведением $A$ на $A^{\prime}$ и обозначим $A \otimes A^{\prime}$ 
множество массивов вида $\left(a, a^{\prime}\right)$, где $a \in A, a^{\prime} \in A^{\prime}$. Из предыдушего ясно, что это снова инвариантное подмножество.

Исходя из аналогии с тензорным произведением представлений, возникают по меньшей мере три вопроса (впрочем, вопросов тут больше, чем ответов). Это ассоциативность, коммутативность и разложение на неприводимые, т.е. на орбиты.

Начнем с ассоциативности, так как тут ответ краток. Очевидно, что мы имеем равенство

$$
\left(A \otimes A^{\prime}\right) \otimes A^{\prime \prime}=A \otimes\left(A^{\prime} \otimes A^{\prime \prime}\right) .
$$

Например, тензорное произведение $O\left(k_{1}\right) \otimes \cdots \otimes O\left(k_{n}\right)$ - это множество всех массивов $I$-веса $k=\left(k_{1}, \ldots, k_{n}\right)$. В частности, если $O$ - произвольная орбита и $k$ - ее вес, то $O$ лежит в $O(k):=O\left(k_{1}\right) \otimes \cdots \otimes O\left(k_{n}\right)$. Это утверждение является аналогом того, что любое неприводимое представление $G L$ есть подпредставление тензорного произведения симметрических степеней стандартного представления.

8.3. Коммутативность. Конечно, $A \otimes A^{\prime}$ не равно $A^{\prime} \otimes A$. Интуитивно ясно, что они должны быть тесно связаны. Однако естественная, казалось бы, биекция, которая отправляет $\left(a, a^{\prime}\right)$ в $\left(a^{\prime}, a\right)$, не является морфизмом, т.е. не согласована с действиями $D$ и $U$. Тем не менее, множества $A \otimes A^{\prime}$ и $A^{\prime} \otimes A$ изоморфныл.

Чтобы показать это, воспользуемся введенным в п. 7.11 изоморфизмом Шютценберже $S c h: A \otimes A^{\prime} \rightarrow *\left(A^{\prime} \otimes A\right)$. Так как для любых $a, a^{\prime}$ верно равенство $*\left(a, a^{\prime}\right)=$ $\left(* a^{\prime}, * a\right)$, мы имеем равенство $*\left(A^{\prime} \otimes A\right)=* A^{\prime} \otimes * A$. Тем самым имеется канонический изоморфизм $S c h: A \otimes A^{\prime} \stackrel{\sim}{\longrightarrow} * A^{\prime} \otimes * A$. Теперь композиция изоморфизмов

$$
A \otimes A^{\prime} \stackrel{S c h \otimes 1}{\longrightarrow} * A \otimes A^{\prime} \stackrel{S c h}{\longrightarrow} * A^{\prime} \otimes A \stackrel{S c h \otimes 1}{\longrightarrow} A^{\prime} \otimes A
$$

дает изоморфизм $C o m: A \otimes A^{\prime} \rightarrow A^{\prime} \otimes A$. Назовем его комьютером.

В связи с этим естественно задать вопрос: будут ли изоморфизмы Sch u/uли Com удовлетворять уравнению Янга-Бакстера? Иначе говоря, будет ли коммутативна диаграмма

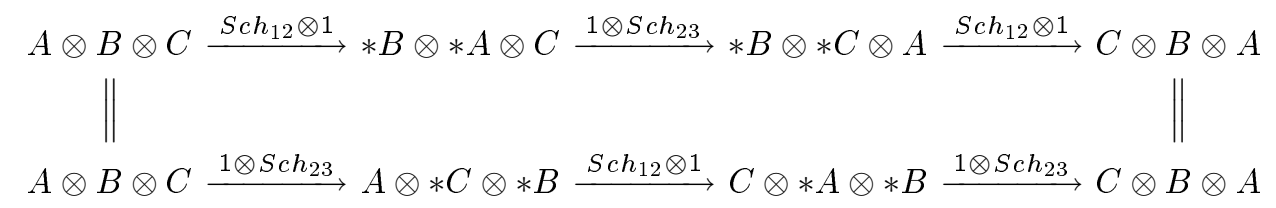

Можно показать, что это так, если алфавиты $I, I^{\prime}$ и $I^{\prime \prime}$ одноэлементные.

8.4. Разложсение на орбиты. Вопрос о разложении на орбиты множества $A \otimes A^{\prime}$ сводится к случаю, когда инвариантные множества $A$ и $A^{\prime}$ сами являются орбитами. Более того, мы будем считать эти орбиты эталонными, т.е. имеюшими вид $O(\mu)$ и $O(\nu)$ для разбиений (или биплотных массивов) $\mu$ и $\nu$.

Как и любое инвариантное множество, $O(\mu) \otimes O(\nu)$ является объединением орбит вида $O(a, b)$, где $a \in O(\mu), b \in O(\nu)$. Кроме того, пару $(a, b)$ можно считать $\mathbf{D}$-плотным массивом. Отсюда видно, что a $\mathbf{D}$-плотный, т.е. фактически совпадает c $\mu$. Что касается $b$, то условие $b \in O(\nu)$ можно переписать как требование, что $b$ является $\mathbf{L}$-плотным массивом формы $\nu$ (или, что то же самое, $I^{\prime}$-веса $\nu$ ). Такую пару $(a, b)$ назовем стандартной парой типа $(\mu, \nu)$; это пара массивов, удовлетворяющая трем требованиям:

1) $a$ - L-плотный массив $I$-веса $\mu$, 
2) $b$ - $\mathbf{L}$-плотный массив $I^{\prime}$-веса $\nu$, и

3) составной массив $(a, b) \mathbf{D}$-плотньй.

Кстати, чему равна форма орбиты $O(a, b)$ ? Ответ: так как $(a, b)$ - D-плотный массив, то его форма равна $J$-весу $(a, b)$.

Зафиксируем итог: орбиты $O(\mu) \otimes O(\nu)$ находятся в биективном соответствии со стандартными парами типа $(\mu, \nu)$.

Обозначим через $S P(\mu, \nu ; \lambda)$ множество стандартных пар $(a, b)$ типа $(\mu, \nu)$ и $J$ веса $\lambda$. (Можно было бы их также обозначить $L R$ от Литтлвуда-Ричардсона.) Тогда множество орбит $O(\mu) \otimes O(\nu)$, изоморфных $O(\lambda)$, отождествляется $c$ $S P(\mu, \nu ; \lambda)$.

Заметим, что похожий ответ имеется и для примера 7.5. Там мы видели, что орбиты инвариантного множества $\mathscr{A}_{\mathbb{Z}}(\lambda \backslash \mu)$ параметризуются $\mathbf{D}$-плотными массивами $a$ такими, что составной массив $\left(\begin{array}{l}a \\ \mu\end{array}\right)$ L-плотньй. Если транспонировать ответ, мы получим множество L-плотных массивов $a^{T}$ таких, что $\left(\mu, a^{T}\right)$ D-плотный массив формы $\lambda$. То есть $S P(\mu, \nu ; \lambda)$, где $\nu$ - форма массива $a^{T}$.

8.5. Биекция коммутативности. Вьше, в п. 8.3, мы построили конкретньй изоморфизм (комьютер) между $O(\mu) \otimes O(\nu)$ и $O(\nu) \otimes O(\mu)$. Поэтому имеется каноническая биекция (комьютер) Com между множествами $S P(\mu, \nu)$ и $S P(\nu, \mu)$. Вспоминая определения, скажем, как она устроена.

Пусть $(a, b)$ - стандартная пара. Сначала мы должны применить инволюцию Шютценберже к массиву $a$. Так как массив $a \mathbf{L}$-плотный, то $S \operatorname{ch}(a)=\mathbf{R} a$ (см. лемму 5.12). Так как массив $(\mathbf{R} a, b)$ D-плотный, то $S c h(\mathbf{R} a, b)$ равен уплотнению вниз перевернутого массива, т.е. $\mathbf{D}(* b, * \mathbf{R} a)=\mathbf{D}(* b, \mathbf{L}(* a))$. Обозначим полученный массив $\left(b^{\prime}, \mathbf{L} a^{\prime}\right)$. Наконец, мы должныприменить $S c h$ к $b^{\prime}$. Так как массив $b$ был $\mathbf{L}$-плотньм, то массивы $* b$ и $b^{\prime}$ являются $\mathbf{R}$-плотными. Поэтому $S c h\left(b^{\prime}\right)=\mathbf{L} b^{\prime}$. И мы получаем окончательно, что $\operatorname{Com}(a, b)=\left(\mathbf{L} b^{\prime}, \mathbf{L} a^{\prime}\right)$, где $\left(b^{\prime}, a^{\prime}\right)=\mathbf{D}(* b, * a)=\mathbf{D} *(a, b)=* \mathbf{U}(a, b)$.

Эта биекция совпадает с переключением таблиц из п. 11.6, а также с биекцией из [14] (и первой фундаментальной биекщией из [44]).

8.6. Биекиия ассоциативности. Аналогично можно рассмотреть разложение на орбиты тройного произведения $O(\mu) \otimes O(\nu) \otimes O(\lambda)$. Они соответствуют стандартньг тройкам $(a, b, c)$, т.е. тройкам массивов, которые удовлетворяют условиям:

a) $a, b, c$ - L-плотные массивы формы (или горизонтального веса) $\mu, \nu$ и $\lambda$ соответственно;

b) массив $(a, b, c) \mathbf{D}$-плотный.

Множество таких троек обозначим $S T(\mu, \nu ; \lambda)$.

По каждой стандартной тройке $(a, b, c)$ можно построить две пары стандартных пар, соответствующие двум способам расстановки скобок в произведении $O(\mu) \otimes$ $O(\nu) \otimes O(\lambda)$.

Сначала мы расставим скобки так: $(O(\mu) \otimes O(\nu)) \otimes O(\lambda)$. Это означает, что мы сначала разлагаем множество $O(\mu) \otimes O(\nu)$ на орбиты $O(a, b)$, а затем каждое произведение $O(a, b) \otimes O(\lambda)$ на орбиты. Это дает нам две стандартные пары $(a, b)$ и $(\mathbf{L}(a, b), c)$. Ясно, что мы получаем биекцию между $S T(\mu, \nu ; \lambda)$ и $\bigsqcup_{\sigma} S P(\mu, \nu ; \sigma) \times S P(\sigma, \lambda)$.

Расставим теперь скобки иначе: $O(\mu) \otimes(O(\nu) \otimes O(\lambda))$. Это дает две другие стандартные пары: $(a, \mathbf{L}(b, c))$ и $\left(b^{\prime}, c^{\prime}\right)$, где $\left(b^{\prime}, c^{\prime}\right)=\mathbf{D}(b, c)$. Стандартность этих пар 
очевидна; отметим только, что вторая пара имеет тип $(\nu, \lambda)$, так как операция $\mathbf{D}$ не меняет $\left(I^{\prime} \sqcup I^{\prime \prime}\right)$-вес. Как и в первом случае, эти две пары связаны соотношением: формы массивов $\mathbf{L}(b, c)$ и $\left(b^{\prime}, c^{\prime}\right)=\mathbf{D}(b, c)$ совпадают. Тем самьм мы построили отображение

$$
S T(\mu, \nu ; \lambda) \rightarrow \bigsqcup_{\tau} S P(\mu, \tau) \times S P(\nu, \lambda ; \tau) .
$$

Тут уже чуть менее очевидно, что это биекция. Но это действительно так. В самом деле, пусть мы имеем элемент правого множества, т.е. две стандартные пары $(a, d)$ и $\left(b^{\prime}, c^{\prime}\right)$, причем форма $d$ совпадает с формой $\left(b^{\prime}, c^{\prime}\right)$. Массив $d$ L-плотный, a $\left(b^{\prime}, c^{\prime}\right)$ - $\mathbf{D}$-плотный. Тогда теорема о биекции 6.2 утверждает, что существует в точности один массив $(b, c)$ такой, что $\mathbf{D}(b, c)=\left(b^{\prime}, c^{\prime}\right)$ и $\mathbf{L}(b, c)=d$.

Собирая все вместе, мы получаем биекцию ассоциативности

$$
\bigsqcup_{\sigma} S P(\mu, \nu ; \sigma) \times S P(\sigma, \lambda) \stackrel{\sim}{\longrightarrow} \bigsqcup_{\tau} S P(\mu, \tau) \times S P(\nu, \lambda ; \tau) .
$$

Ее можно переписать также в виде

$$
S P(\mu, \nu) \times_{\Lambda} S P(\cdot, \lambda) \stackrel{\sim}{\longrightarrow} S P(\mu, \cdot) \times_{\Lambda} S P(\nu, \lambda) .
$$

Эта форма удобна тем, что верна для любых $\mu, \nu, \lambda$, а не только для целочисленных разбиений. При этом сомножители расслоенных произведений являются полиэдрами, как и сами расслоенные произведения. Однако стоит предупредить, что биекция ассоциативности не линейна, а только кусочно линейна.

8.7. ЗАмЕчАнИЕ. При функциональном взгляде на массивы множество $S P(\mu, \nu ; \lambda)$ отождествляется с множеством дискретно вогнутых функций на треугольном гриде размера $m$, которые вдоль сторон имеют прирашения $\mu, \nu$ и $\lambda$. Можно показать, что биекция ассоциативности совпадает с биекцией, построенной в работе [36] с помошњю так называемой октаэдральной рекурсии. Однако введение этого языка и изложение деталей доказательства потребовало бы слишком много места.

\section{Часть II. Таблицы Юнга}

\section{9. Словесное представление массивов}

9.1. Мы уже говорили в п. 2.5, что D-плотные целочисленные массивы соответствуют полустандартньм таблицам Юнга. С другой стороны, ясно, что даже если мы интересуемся только полустандартными таблицами, т.е. D-плотными массивами, то на промежуточных этапах удобно использовать произвольные массивы. Не зная массивов, традищионная теория нашла два способа работать с этими промежуточными объектами. Один способ - работать со словами (или текстами, см. ниже). Второй - работать с косьми таблицами. Начнем с первого, частично напоминая сказанное в п. 2.4 .

Рассмотрим сначала однострочный (целочисленньй) массив. Это просто последовательность $a=(a(1), \ldots, a(n))$ натуральных чисел. Свяжем с ним слово $w(a)$ в алфавите $I=\{1, \ldots, n\}: w(a)=1^{a(1)} \ldots n^{a(n)}$. Например, массиву $a=(0,2,1,0,1,2,0)$ 
соответствует слово $w(a)=223566$. В этом слове буквы идут в (нестрого) возрастающем порядке. Такие монотонные слова будем назьвать словами-строками. Ясно, что слова-строки взаимно однозначно соответствуют однострочным массивам.

Перейдем теперь к массивам из $m$ строк. Такой массив представляет $m$ однострочных массивов $a(\cdot, 1), \ldots, a(\cdot, m)$, поставленных друг на друга. С каждым массивом $a(\cdot, j)$ связано слово-строка $w(j)=w(a(\cdot, j))$. Естественно массиву $a$ сопоставить слово $w(m) \ldots w(1)$. Однако чтобы можно было однозначно выделять строки их этого сплошного слова (и тем самым восстанавливать исходный массив), удобно разделять слова-строки $w(j)$ вспомогательным символом-разделителем ·. Такую последовательность слов-строк, разделенных символом ·, назовем текстом. Теперь тексты биективно соответствуют массивам с неопределенным числом строк. Например, текст $\cdot 24 \cdot 1335$ соответствует массиву

\begin{tabular}{|l|l|l|l|l|l|}
\hline 0 & 0 & 0 & 0 & 0 & 0 \\
\hline 0 & 1 & 0 & 1 & 0 & 0 \\
\hline 0 & 0 & 0 & 0 & 0 & 0 \\
\hline 1 & 0 & 2 & 0 & 1 & 0 \\
\hline
\end{tabular}

Пользуясь язьком текстов, мы можем не упоминать про массивы. Надо понимать, однако, что при этом нарушается симметрия между алфавитами $I$ и $J$, что приводит к разным неудобствам.

9.2. Пусть у нас есть два слова-строки $w=w_{1} \ldots w_{s}$ и $v=v_{1} \ldots v_{t}$. Скажем, что $v$ доминирует $w$, если $t \leqslant s$ и если для любого $k \leqslant t$ вьполнено $w_{k}<v_{k}$. То есть если мы запишем слово $w$ под словом $v$, мы получаем строгое возрастание букв по вертикали.

Текст $w(m) \cdot \ldots \cdot w(1)$ назьвается таблицей (или, чтобы не путаться с таблицами Юнга, словом-таблицей), если каждое слово $w(j+1)$ доминирует следующее слово $w(j)$. Очевидно, что это просто другой способ говорить о полустандартных таблищах. Из сказанного в п. 2.5 видно, что слова-таблицы биективно соответствуют D-плотным массивам. Зафиксируем это как следующее предложение.

9.3. ПРЕДЛОЖЕНИЕ. Пусть а-челочисленный массив. Эквивалентны утверждения:

1) массив а является D-плотным ;

2) текст $w($ а) является словом-таблицей;

3) соответствующая “таблица" Т(а) является полустандартной таблиией Юнга.

Отметим еше, что “форма” $Y(a)$ таблицы $T(a)$ совпадает с формой массива $a$. В самом деле, таблица $T(a)$ вписана в диаграмму Юнга $Y(a)$, у которой в $j$-й строке имеется $l(j)$ клеток, где $l(j)$ - длина слова $w(j)$, т.е. $w_{j}(a)$. То есть соответствуюшее разбиение $\lambda=(l(1), \ldots, l(m))$ - это $J$-вес $w_{J}(a)$. Но для $\mathbf{D}$-плотного массива $a$ его форма равна $J$-весу.

9.4. Часто используют еще одну интерпретацию полустандартных таблиц. Рассмотрим те квадратики в $Y(a)$, в которых стоит символ $n \in I$, и удалим их из диаграммы $Y(a)$. Ясно, что мы получим форму $Y\left(a^{\prime}\right)$ массива $a^{\prime}$, полученного выбрасыванием последнего $n$-го столбца массива $a$. Диаграмма $Y\left(a^{\prime}\right)$ вписана в диаграмму 
$Y(a), Y\left(a^{\prime}\right) \subset Y(a)$. Но этого мало. Разность $Y(a) \backslash Y\left(a^{\prime}\right)$ является так назьваемой горизонтальной полосой, т.е. по вертикали может стоять не более одной клетки.

Эту процедуру можно продолжать, выбрасьвая по очереди клетки с символами $n-1, \ldots, 1$. В результате мы получим фильтрацию диаграмм Юнга

$$
\varnothing=Y_{0} \subset Y_{1} \subset \cdots \subset Y_{n}=Y(a) .
$$

При этом все разности $Y_{i+1} \backslash Y_{i}$ являются горизонтальными полосками. Обратно, если имеется такая фильтрация, мы можем заполнить каждую разность $Y_{i+1} \backslash Y_{i}$ символом $i+1$ и получим полустандартую таблицу или $\mathbf{D}$-плотньй массив $a$. При этом диаграмма $Y_{i}$ - это форма массива $a_{i}$, полученного из а удалением столбцов $i+1, \ldots, n$.

С этой точки зрения полустандартная таблица - это некоторьй специальньй монотонньй путь в множестве диаграмм Юнга.

9.5. Паттерны Гельфанда-Цетлина. Вьше мы предполагали, что D-плотный массив $a$ целочисленный. Однако целочисленность нужна была только для того, чтобыговорить о словах-текстах или полустандартных таблицах. Теперь мы можем ввести терминологию, пригодную для любого массива. А именно, назовем дробной формой (с $m$ частями) убываюшую последовательность неотрицательных вещественных чисел $\lambda=(\lambda(1) \geqslant \cdots \geqslant \lambda(m))$. Для двух таких форм $\lambda$ и $\lambda^{\prime}$ мы пишем $\lambda^{\prime} \subset \lambda$, если $\lambda(j) \geqslant \lambda^{\prime}(j)$ для любого $j$. Скажем, что разность $\lambda \backslash \lambda^{\prime}$ элементарна, если

$$
\lambda(1) \geqslant \lambda^{\prime}(1) \geqslant \lambda(2) \geqslant \lambda^{\prime}(2) \geqslant \cdots
$$

(иначе говоря, $\lambda$ и $\lambda^{\prime}$ перемежаются).

Убываюшая последовательность $\lambda_{1} \supset \cdots \supset \lambda_{n}$ дробных форм назьвается namтерном Гельфанда-Цетлина, если все разности $\lambda_{i} \backslash \lambda_{i-1}$ элементарны. Тогда $\mathbf{D}$ плотньй массив - это то же самое, что элементарная фильтрация "дробных" диаграмм Юнга.

Начальная форма $\lambda_{1}$ назьвается формой паттерна Гельфанда-Цетлина; конечно, это то же самое, что форма соответствующего $\mathbf{D}$-плотного массива. Множество $G C(\lambda)$ паттернов Гельфанла-Цетлина фиксированной формы $\lambda$ является многогранником, как видно из приведенных выше неравенств перемежаемости. Структура этого многогранника исследовалась в работах $[33],[16],[9]$.

9.6. Стоит сказать и про словесную интерпретацию $\mathbf{L}$-плотных массивов. Если массив $a \mathbf{L}$-плотный, то для любого шара в клетке $(i, j)$ (т.е. буквы $i$ в $j$-м слове текста $w(a))$ существует его “левый” партнер в клетке $\left(i-1, j^{\prime}\right), j^{\prime}<j$ (т.е. буква $i-1$ в некотором следуюшем слове нашего текста). Иначе говоря, для каждого вхождения буквы $i$ есть свое вхождение буквы $i-1$, расположенное правее. Такие слова (или тексты) назьваются словами Я Я манучи в [21], [38] (или обратныци решетчатыми словами в [40]). Слово $w$ назьвается словом Яманучи, если для любого его суффикса $v$ вес (конечно, $I$-вес) этого слова $v$ образует убывающую последовательность (т.е. является разбиением). В частности, если мы обрежем наш массив до массива $a \leqslant k$, оставив только $k$ нижних строк, вес этого массива $a^{\leqslant k}$ по-прежнему будет убываюшей последовательностью, и так для любого $k$.

Конечно, верно и обратное. 


\section{7. ПРЕДЛОЖЕНИЕ. Эквивалентны утверждения:}

1) массив а L-плотный

2) текст $w($ а) является словом Яманучи.

ЗАмечанИЕ. Симметричные понятия $\mathbf{D}$-плотности и $\mathbf{L}$-плотности на языке слов вьглядят совершенно по-разному - одно соответствует понятию таблищы (как текста), а другое - понятию слова Яманучи.

9.8. Наконец, если массив $a$ биплотньй, т.е. одновременно $\mathbf{D}$-плотньй и $\mathbf{L}$-плотньй, его слово-текст $w(a)$ является одновременно таблицей и словом Яманучи. Это значит, что $i$-я строка этого слова состоит из одних букв $i$ (в количестве $a(I, i)$ ).

Можно повторить, что биплотньй (целочисленный) массив - это просто разбиение (форма). А произвольньй - дробная форма.

\section{0. Эквивалентность Кнута}

10.1. Итак, вместо массивов (формата $I \times\{1,2, \ldots\})$ можно работать с текстами (в алфавите $I$ ). Основной структурой для массивов были операции $D$ и $U$. Частичной заменой операций $D$ и $U$ на языке текстов служит эквивалентность Кнута.

Кнут [34] (см. также [19]) задавал свою эквивалентность двумя соотношениями, которые мы сейчас приведем. Однако так как мы используем еще разделитель ·, приходится ввести еше одно тривиальное соотношение, с которого мы и начнем. Далее $x, y$ и $z$ обозначают символы из алфавита $I$, упорядоченные так, что $x \leqslant y \leqslant z ; u$ и $v-$ произвольные тексты.

0) Текст $u x \cdot y v$ эквивалентен тексту $u \cdot x y v$ (а также тексту $u x y \cdot v$ ). Кроме того, $u \cdot v$ эквивалентен $u \cdot v$.

А теперь две собственно кнутовские эквивалентности:

1) если $x \leqslant y<z$, то эквивалентны тексты

$u x z \cdot y v$ и $u z \cdot x y v$

2) если $x<y \leqslant z$, то эквивалентны тексты

$u y z \cdot x v$ и $и y \cdot x z v$.

10.2. ОПРЕДЕЛЕНИЕ. Эквивалентность Кнута - это отношение эквивалентности на текстах, порожденное соотношениями 0)-2).

Очевидно, что эквивалентность Кнута согласована с естественным умножением (конкатенацией) текстов. Плактическим моноидом называется фактормоноид свободного моноида, порожденного $I$ и ·, по отношению эквивалентности Кнута. Единичным элементом этого моноида служит пустое слово (а также любое слово вида …).

10.3. ТеОрема. Пусть а и по Кнуту тогда и только тогда, когда массивы а и относительно действий $D$ и $U$.

Доказательство этой теоремы приводится в следующих двух пунктах. 
10.4. Проверим сначала, что элементарные эквивалентности 0)-2) представляют не что иное, как применение операций $D$ (или $U$ ) к соответствуюшим массивам. Начнем с простейшего случая 0$)$. Текст $* x \cdot y *$ соответствует массиву

$$
\begin{array}{llllll}
* & * & * & x & 0 & 0 \\
0 & 0 & 0 & y & * & *
\end{array} .
$$

(Мы выделили из всего массива только две строчки, соответствующие разделителю · Символ $y$ располагается ровно под $x$, если $x=y$. Если $x<y$, под $x$ стоит 0 , а шар $y$ располагается правее.) Операция $D$ (с соответствуюшим номером) опускает шар $x$ вниз, превращая массив в

$$
\begin{array}{cccccc}
* & * & * & 0 & 0 & 0 \\
0 & 0 & 0 & x y & * & *
\end{array},
$$

которьй и соответствует тексту $* \cdot x y *$.

Рассмотрим теперь эквивалентность 1$)$. Первое слово $* x z \cdot y *$ соответствует массиву (снова мы рисуем только две соответствующие строчки)

$$
\begin{array}{llllllll}
* & * & x & 0 & 0 & z & 0 & \\
0 & 0 & 0 & y & * & * & * & \text {. }
\end{array} \text {. }
$$

Символ $y$ располагается под $x$ или правее, но точно левее $z$. Когда мы образуем стабильный матчинг между этими строками, шар $z$ точно получает себе партнера ( $y$ или кого-то еше ближе). Поэтому самым правым свободньм шаром в верхней строчке будет $x$. Значит, операция $D$ перемешает $x$ вниз, и мы получаем массив

$$
\begin{array}{lllllll}
* & * & 0 & 0 & 0 & z & 0 \\
0 & 0 & x & y & * & * & *
\end{array},
$$

как раз соответствующий второму слову.

Аналогично разбирается эквивалентность 2$)$. Второму слову $* y \cdot x z *$ соответствует массив

$$
\begin{array}{lllllll}
* & * & * & y & 0 & 0 & 0 \\
0 & 0 & x & 0 & z & * & *
\end{array} .
$$

Шар $x$ получает себе партнера наверху ( $y$ или что-то ближе), шар $z$ - нет. Поэтому $z-$ самьй левый свободный шар в нижней строчке, и операция $U$ поднимает его наверх. Мы получаем массив

$$
\begin{array}{lllllll}
* & * & * & y & z & 0 & 0 \\
0 & 0 & x & 0 & 0 & * & *
\end{array},
$$

которьй соответствует первому слову $* y z \cdot x *$.

10.5. Мы знаем, что, применяя операции $D_{j}$ достаточное число раз, мы преврашаем любой массив в $\mathbf{D}$-плотный. Плактическим аналогом этого служит следующее утверждение: применяя эквивалентности 0)-2), можно любой текст превратить в текст-таблицу. Это будет доказывать теорему 10.3 .

Ключом к доказательству этого утверждения служит операция вставки (insertion, bamping) буквы в строку. Пусть $u$ - некоторое слово-строка и $l$-некоторая буква из $I$. Утверждается, что текст вида $u \cdot l$ эквивалентен тексту вида $l^{\prime} \cdot u^{\prime}$ (или $u^{\prime}$ ), где $l^{\prime}-$ буква, а $u^{\prime}$ - строка. 
Мы оставляем читателю проверку этого нехитрого утверждения. Вместо этого мы покажем, что происходит при этом на уровне массивов. Текст $u \cdot l$ представляется двухстрочным массивом

$$
\begin{array}{llllll}
* & * & * & * & * & * \\
0 & 0 & l & 0 & 0 & 0
\end{array}
$$

(верхняя строка представляет слово $u$, нижняя - букву $l$ ). Если правее $l$ в верхней строке нет ничего, мы имеем случай тривиальной перестройки 0). Применение операции $\mathbf{D}=D^{\infty}$ опускает всю верхнюю строку, преврашая массив в

$$
\begin{array}{llllll}
0 & 0 & 0 & 0 & 0 & 0 \\
* & * & l & 0 & 0 & 0
\end{array} .
$$

В общем случае $l$ находит в верхней строке партнера $l^{\prime}$, так что наш массив имеет вид

$$
\begin{array}{llllll}
* & * & * & 0 & l^{\prime} & * \\
0 & 0 & l & 0 & 0 & 0
\end{array}
$$

(заметим, что в промежутке между $l$ и $l^{\prime}$ ничего нет). Снова применяем $D$ много раз. Вначале опускаются шары, стояшие правее $l^{\prime}$ (перестройка 2)). Затем опускаются все шары, стоящие левее $l^{\prime}$ (перестройка 1$)$ ). И в результате получается массив

$$
\begin{array}{lllllll}
0 & 0 & 0 & 0 & l^{\prime} & 0 & \\
* & * & l & 0 & 0 & * & .
\end{array} .
$$

Оглядываясь на проделанное, мы видим, что операция вставки буквы $l$ в строку $u-$ это применение операции $\mathbf{D}=D^{\infty}$. А операция Шенстеда-Кнута вставки буквы в текст-таблицу - это применение операции $\mathbf{D}_{m} \ldots \mathbf{D}_{1}$. Так что “элементарньй” шаг алгоритма вставки - это довольно длинная серия истинно элементарных операций $D_{j}$.

10.6. Вьше мы показали, что эквивалентные (по Кнуту) тексты соответствуют массивам, лежашим в одной орбите (относительно операторов $D$ и $U$ ). Кроме того, каждый текст $w$ эквивалентен таблице. Согласно сказанному вьше, эта таблица определена однозначно. Традиционно она обозначается $P(w)$. Из сказанного вьше ясно также, что

$$
P(w(a))=w(\mathbf{D}(a))
$$

То есть что эта таблица - это в точности таблица, соответствующая $\mathbf{D}$-плотному массиву $\mathbf{D} a$.

10.7. Кроме эквивалентности по Кнуту на множестве текстов можно рассматривать так называемую дуальную (или коплактическую) эквивалентность. Мы не приводим точного определения, потому что оно звучит не слишком естественно на языке слов-текстов. Но понятно, что на языке массивов это соответствует орбитам относительно горизонтального действия $L-R$. Чуть подробнее это написано в [38]. 


\section{1. Косые таблицы}

11.1. Второй способ обходиться без массивов - использование так назьваемых коcblx полустандартных таблиц. Такая таблица размещается в “косой” диаграмме Юнга. Так называют разность $\lambda \backslash \mu$ двух "прямых" диаграмм $\lambda$ и $\mu$ таких, что $\mu \subset \lambda$. Заполнение такой диаграммы символами из алфавита $I$ назьвается полустандартной таблицей (“формы” $\lambda \backslash \mu$ ), если в строках символы (нестрого) возрастают, а в столбцах - возрастают строго.

Если мы, как обычно, прочитаем строки такой таблицы, мы получим массив. Тут все как в пाп. 2.4-2.6). Обратно, если у нас дан массив $a$, мы можем представить его косой таблищей. Косой, потому что "прямые" таблицы соответствуют, как мы знаем, D-плотным массивам. Нужно сдвинуть строки, соответствуюшие словам $w(a(\cdot, j))$, чтобы добиться строгого возрастания в столбцах. Например, пусть массив $a$ имеет вид

$$
\begin{array}{lllll}
0 & 1 & 0 & 0 & 3 \\
0 & 0 & 0 & 1 & 0 \\
2 & 0 & 0 & 0 & 0
\end{array} .
$$

Он дает таблоид

$$
\begin{array}{llll}
2 & 5 & 5 & 5 \\
4 & & & \\
1 & 1 & &
\end{array}
$$

Это не полустандартная таблища Юнга. Однако, сдвигая вправо строки, можно превратить это в косую полустандартную таблицу. Например, такую:

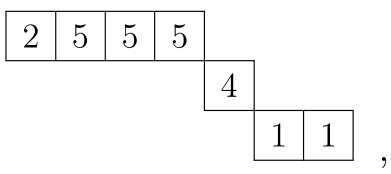

или в “более экономную"

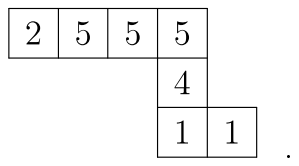

11.2. Кстати, на сколько нужно сдвигать строки? Ответ уже был дан в п. 3.2, где мы ввели понятие дефицита. Более точно, обозначим через $\delta_{j}$ дефицит между строками $j$ и $j+1$ нашего массива. Тогда при построении диаграммы мы должны сдвинуть вправо $j$-ю строку диаграммы как минимум на $\delta_{j}$ относительно $(j+1)$-й строки (самую верхнюю строчку можно совсем не сдвигать). Таким образом, при минимальном, или экономном, сдвиге диаграмма $\mu$ для нашей формы $\lambda \backslash \mu$ имеет вид: $\mu(1)=\delta_{1}+\cdots+\delta_{m-1}$, $\ldots, \mu(m-1)=\delta_{m-1}$. Или: $\mu(m)=0$ и $\mu(j)=\delta_{j}+\mu(j+1)$ при $j<m$.

Конечно, "внешняя форма" $\lambda$ нашей диаграммы равна $\mu+w t_{J}(a)$.

Видно, что такое представление массивов, как и словесное, страдает односторонностью. Кроме того, как мы видели, несколько косых таблиц могут соответствовать одному и тому же массиву. Тем не менее это достаточно гибкая форма. 
11.3. Jeu de taquin. Частичную замену операциям $D_{j}$ доставляет операция jeu de taquin, придуманная М. П. Шютценберже (см. [39], [52] и [47]). Применяя эту операцию нужное число раз, мы косую таблицу преврашаем в "прямую” полустандартную таблицу. На языке массивов это соответствует переходу от массива $a$ к массиву $\mathbf{D} a$.

Опишем элементарньй шаг этой операции. Пусть у нас есть косая таблица $T$ формы $\lambda \backslash \mu$. Возьмем угловую клеточку диаграммы $\mu$. Справа и сверху ее ограничивают клеточки нашей таблицы. Переместим в эту пустую клетку символ из соседней “табличной” клетки (соответственно, “пустой” становится та клетка, из которой символ ушел). Правило тут такое - наша пустая клетка меняется с той клеткой (справа или сверху), в которой стоит меньший символ; если оба соседних символа равны, меняется с верхней клеткой. Новая пустая клетка меняется со своими соседями и так далее до тех пор, пока у нее есть соседи справа или сверху.

В результате такого слайдинга (или эвакуации) пустой клетки мы получаем новую таблицу формы $\lambda^{\prime} \backslash \mu^{\prime}$, у которой и внешняя диаграмма $\lambda^{\prime}$, и внутренняя $\mu^{\prime}$ уменьшились на одну клетку. В конще конщов мы эвакуируем все пустые клетки из диаграммы $\mu$ и получим нормальную прямую таблицу. Ее обозначают как $J e u(T)$ или $T^{E}$ (от слова evacuation). Может показаться, что результирующая таблища $J e u(T)$ зависит от порядка эвакуации пустых клеток, но это не так.

Чтобы убедиться в этом, рассмотрим массивный смысл операции эвакуации.

11.4. Удобно превратить нашу косую таблицу формы $\lambda \backslash \mu$ в прямую, вставив на пустые клетки диаграммы $\mu$ фиктивные, или вспомогательные, символы $0,-1,-2, \ldots$ Вставлять можно как угодно, лишь бы получившаяся таблица формы $\mu$ была полустандартной (тогда автоматически “расширенная" таблица формы $\lambda$ будет полустандартной). Например, можно заполнить ее словом Яманучи.

Фактически мы тавтологическим способом преврашаем наш массив $a$ в $\mathbf{D}$-плотньй. Если в исходном массиве $a$ был свободный шар (в строке $j+1)$, мы добавляем в ящик $(0, j)$ фиктивньй шар. Добавив так нужное число шаров в столбец 0 , мы найдем партнеров для всех старых свободных шаров в массиве $a$. Однако в новом массиве шары в столбце 0 станут свободными (кроме самого нижнего яшика $(0,1)$ ). Добавим для них фиктивных партнеров в столбце -1 и т. д. В результате мы получаем "экономное" продолжение нашего массива $a$ до $\mathbf{D}$-плотного массива $\widetilde{a}$ (конечно, при этом к алфавиту $I$ добавляются фиктивные символы $-m+1, \ldots,-1,0)$. Более формально, расширенньй массив $\widetilde{a}=\left(a^{\prime}, a\right)$ есть конкатенация вспомогательного массива $a^{\prime}$ формата $\{-m+1, \ldots,-1,0\} \times J$ и массива $a$. По построению $\widetilde{a} \mathbf{D}$-плотньй, откуда, конечно, следует, что $a^{\prime}$ тоже $\mathbf{D}$-плотньй.

Приступим теперь к эвакуации. Мы берем некоторьй фиктивньй шар $b$ и удаляем его. Пусть он находился в строчке $j$. Возможны два случая. Либо этот шар $b$ не был опорой ни для какого шара из строки $j+1$. Тогда его удаление не сказывается на плотности массива. В таблице это скажется только в сдвиге (на единицу влево) всех символов, стояших вправо от $b$. То есть наша дыра движется вправо до конща строки. Либо (второй случай) наш шар $b$ был опорой (партнером) для некоторого шара $b^{\prime}$ на этаже $j+1$. Лишенньй опоры, этот шар $b^{\prime}$ опускается на этаж ниже (и там уже обретает опору). Однако опускание этого шара может лишить опоры некоторый шар $b^{\prime \prime}$, которьй опускается вниз, и т. д.

Тут важно отметить, что когда лишается поддержки очередной шар из массива $a$, то он является самым правьм свободным шаром в своем ряду. И его опускание - это 
в точности применение операции $D$ с соответствующим номером.

Таким образом, эвакуация одной пустой клеточки соответствует (в терминах исходного массива) применению операторов $D_{j}, D_{j+1}, \ldots, D_{k}$, где $j$ - это номер строки, в которой расположена начальная дыра, a $k+1$ - номер строки, где эта дыра исчезает, пройдя таблицу насквозь. А эвакуация всех пустых клеток (или всех клеток с фиктивными символами) - это применение $\mathbf{D}$ к исходному массиву $a$.

11.5. С помощью введенной выше операции эвакуации вводится понятие эквивалентности по Кнуту косых таблиц. Таблищы эквивалентны по Кнуту, если они приводятся к одной и той же прямой таблице. Или, что то же самое, если они получаются друг из друга применением нескольких операций jeu de taquin. Конечно, в терминах массивов это означает, что массивы имеют одинаковое $\mathbf{D}$-уплотнение, или что они лежат в одной орбите.

Симметрично (но менее естественно) вводится и дуальная эквивалентность косых таблиц, см. [27]. Разумеется, дуальная эквивалентность в терминах массивов означает, что массивы имеют одинаковые L-уплотнения. Как отмечено в [4], глубочайший результат М. Хаймана состоит в том, что класс эквивалентности по Кнуту и класс дуальной эквивалентности пересекаются не более чем по одному элементу. Конечно, это следует из нашей теоремы о биекции 6.2.

11.6. Интересное использование процедуры эвакуации было предложено в [4]. А именно, вместо того, чтобы просто эвакуировать пустую клетку, можно перевозить на ней записанньй в нее символ. Допустим, мы, как в п. 11.4, заполнили диаграмму $\mu$ символами из вспомогательного алфавита $I^{\prime}=\{-m, \ldots,-1,0\}$, так что внутри диаграммы $\mu$ получается таблища $T^{\prime}$. Символы из $I^{\prime}$ меншше символов из $I$. Теперь возьмем и прибавим к числам из $I^{\prime}$ "большое" число (например, $N>n+m$ ). Все символы из $I^{\prime}$ станут больше символов из $I$ и полустандартность нарушится. Начнем теперь по очереди эвакуировать символы из диаграммы $\mu$, перенося на них написанный символ. В результате мы получим новую прямую таблицу формы $\nu \subset \lambda$, заполненную символами из $I$, и косую таблицу формы $\lambda / \nu$, заполненную символами из $I^{\prime}+N$.
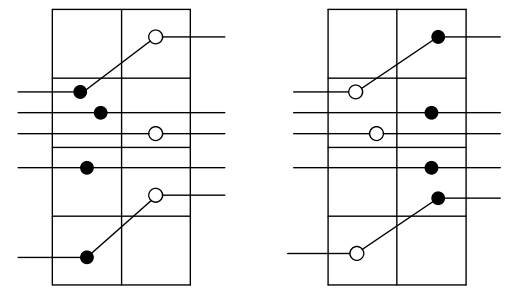

Рис. 9.

Массивньй смысл этой операции такой. Пусть у нас есть два массива, $a$ и $a^{\prime}$ (с горизонтальными алфавитами $I$ и $\left.I^{\prime}, I<I^{\prime}\right)$, и мы образовали составной массив $\left(a, a^{\prime}\right)$. Тогда можно сделать “переключение” этих массивов, т.е. построить новый составной массив $\left(b^{\prime}, b\right)$, причем

1) дефициты (и эксцессы) у $\left(a, a^{\prime}\right)$ те же, что и у $\left(b^{\prime}, b\right)$;

2) $I$-вес $a$ равен $I$-весу $b$;

3) $I^{\prime}$-вес $a^{\prime}$ равен $I^{\prime}$-весу $b^{\prime}$. 
Идею этой конструкции можно продемонстрировать на примере, где мы переключаем только два соседних столбца (см. рис. 9).

\section{2. Правило Литтлвуда-Ричардсона}

12.1. Функции Шура. Хотя числа (или коэффищиенты) Литтлвуда-Ричардсона появляются в различных теориях, проще всего их определить в терминах многочленов Шура. Пусть $a$ - массив из $m$ строчек. Будем понимать его $J$-вес мультипликативно. То есть сопоставим этому массиву следуюший моном от переменных $x_{1}, \ldots, x_{m}$, $x^{w t_{J}(a)}=x_{1}^{w_{1}(a)} \cdots x_{n}^{w_{n}(a)}$, которьй для краткости обозначают как $x^{a}$.

Если $A$ - некоторое конечное множество целочисленных массивов, то можно рассмотреть $s(A)$, сумму всех мономов $x^{a}, a \in A$. Это Элемент кольца многочленов $\mathbb{Z}\left[x_{1}, \ldots, x_{m}\right]$. Если множество $A$ инвариантно (относительно $D U$-действий, см. раздел 7$)$, можно показать, что этот многочлен $s(A)$ симметричен (это сразу не очевидно, но см., например, дополнение А).

Нас особенно будет интересовать случай, когда $A=O$-орбита, и даже эталонная орбита $O(\lambda)$, где $\lambda$ - разбиение с $m$ частями. Многочлен $s(O(\lambda))$ обозначается $s_{\lambda}$ и называется многочленом (или функиией) Шура. ${ }^{2}$

Орбита $O(\lambda)$ состоит из всевозможных L-плотных массивов $a$ формы $\lambda$. Если транспонировать $a$, мыполучаем $\mathbf{D}$-плотный массив $a^{T}$, или, согласно п. 9.3, полустандартную таблицу $T\left(a^{T}\right)$ формы $\lambda$. Вес этой таблицы равен $J$-весу $a$. Число полустандартных таблиц формы $\lambda$ и веса $\mu$ назьвается иислом Костки и обозначается $K_{\lambda, \mu}$. В этих терминах

$$
s_{\lambda}=\sum_{\mu} K_{\lambda, \mu} x^{\mu},
$$

где $\mu$ пробегает $\mathbb{Z}_{+} \otimes J=\mathbb{Z}_{+}^{m}$. При этом полезно иметь в виду, что число Костки $K_{\lambda, \mu}$ отлично от нуля тогда и только тогда, когда вектор $\lambda$ мажорирует вектор $\mu$ (см. [42], [21], [9]).

12.2. ПРимеР (см. пример $7.7, \mathrm{~d})$ ). Пусть $J=\{x, y, z\}$ и $\lambda=(2,1)$. Орбита $O(\lambda)$ состоит из 8 массивов. Соответствуюшая функция Шура $s_{\lambda}$ равна

$$
S_{(2,1)}=x x y+x x z+x y y+2 x y z+y y z+x z z+y z z .
$$

Коэффищиент 2 при $x y z$ объясняется тем, что два массива нашей орбиты имеют вес $(1,1,1)$.

12.3. ПримеР. Пусть $O(k)$ - множество всех (одностолбцовых) массивов веса $k$. Соответствуюший многочлен Шура $s_{(k)}$ есть не что иное, как полный симметрический многочлен $h_{k}$ степени $k$ (от переменных $x_{1}, \ldots, x_{m}$ ).

\footnotetext{
${ }^{2}$ Моном $x^{a}$ определен для произвольного массива $a$. Рассмотрим непрерывную орбиту $O^{\mathbf{R}}(\lambda)$ применяя произвольные степени операций $D_{j}^{t}, U_{j}^{t}, t \geqslant 0$. Соответствующая “сумма" мономов станет интегралом Шура
}

$$
\mathscr{S}_{\lambda}\left(x_{1}, \ldots, x_{n}\right)=\frac{\sum_{\sigma \in S_{n}} \operatorname{sign}(\sigma) x^{\sigma(\lambda)}}{\prod_{i<j}\left(\ln x_{i}-\ln x_{j}\right)} .
$$


Для вектора $\mu=\left(\mu_{1}, \ldots, \mu_{n}\right) \in \mathbb{Z}_{+}^{m}$ через $h_{\mu}$ обозначают произведение $h_{\mu_{1}} \cdots h_{\mu_{n}}$. Конечно, это многочлен $s(O(\mu))$, где $O(\mu)=O\left(\mu_{1}\right) \otimes \cdots \otimes O\left(\mu_{n}\right)$. Разложение этого множества на орбиты (см. п. 8.4) дает формулу

$$
h_{\mu}=\sum_{\lambda} K_{\lambda, \mu} s_{\lambda} .
$$

Отсюда легко получить, что многочлены Шура дают $\mathbb{Z}$-базис кольца симметрических многочленов (см. также [40]). А обрашение этой формулы приводит к детерминантной формуле Якоби для функций Шура.

12.4. Так как функции Шура образуют аддитивный базис кольца симметрических многочленов, то произведение функций Шура линейно выражается через функции Шура. Иначе говоря,

$$
s_{\mu} s_{\nu}=\sum_{\lambda} c_{\mu, \nu}^{\lambda} s_{\lambda}
$$

для некоторых целых чисел $c_{\mu, \nu}^{\lambda}$. Эти числа назьваются коэффициентами Литтлвуда-Ричардсона, потому что Литтлвуд и Ричардсон дали комбинаторное описание этих чисел.

Чтобы привести это описание, вспомним, что функция $s_{\mu}$ соответствует орбите $O(\mu)$ (можно даже назвать ее характером этой орбиты), а $s_{\nu}$ - орбите $O(\nu)$. Очевидно, что произведение $s_{\mu} s_{\nu}$ соответствует тензорному произведению $O(\mu) \otimes O(\nu)$. В самом деле, $w t_{J}\left(a, a^{\prime}\right)=w t_{J}(a)+w t_{J}\left(a^{\prime}\right)$, откуда $x^{\left(a, a^{\prime}\right)}=x^{a} x^{a^{\prime}}$. Разложение вьше соответствует разложению произведения на орбиты. Коэффициент $c_{\mu, \nu}^{\lambda}$ указывает, сколько раз в этом разложении встречается орбита, изоморфная $O(\lambda)$. Как раз этой задачей мы занимались в п. 8.4. Там мы не только нашли это число, но и идентифицировали орбиты. А именно, множество орбит, изоморфных $O(\lambda)$, отождествляется с множеством $S P(\mu, \nu ; \lambda)$ стандартных пар $(a, b)$ типа $(\mu, \nu)$, у которых $J$-вес равен $\lambda$. И нам остается только перевести этот ответ на язык таблиц. Ответ вьглядит так.

12.5. Теорема (правило Литтлвуда-Ричардсона). Коэффициент $c_{\mu, \nu}^{\lambda}$ равен числу косых полустандартных таблии $T$ формы $\lambda \backslash \mu$ и веса $\nu$, у которых соответствующее слово $w(T)$ является словом Яманучи.

В самом деле, стандартная пара $(a, b)$, являясь $\mathbf{D}$-плотным массивом, дает полустандартную таблицу формы, равной $J$-весу $(a, b)$, т.е. $\lambda$. Биплотньй подмассив $a$ дает подтаблицу формы $\mu$, которая нас в данный момент не интересует. Оставшаяся косая часть таблицы $\lambda \backslash \mu$ заполнена словом $w(b)$, которое в силу $\mathbf{L}$-плотности массива $b$ является словом Яманучи. Ясно, что все это обратимо.

Одно замечание. Из коммутативности умножения в кольце симметрических функций видно, что $c_{\mu, \nu}^{\lambda}=c_{\nu, \mu}^{\lambda}$. В п. 8.5 эта симметрия объясняется на орбитном уровне.

12.6. ПримеР. Рассмотрим конкретньй пример. Пусть снова $m=3, \mu=(1), \nu=$ $(1,1)$. Будем действовать согласно правилу 12.5 . Непосредственно можно убедиться, что имеются 4 косые таблищы формы ? \(1) и веса $\nu=(1,1)$. Это

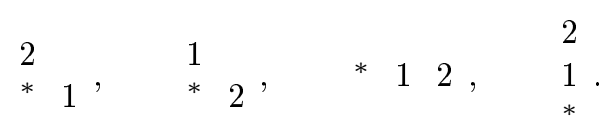


Из них первая и четвертая дают слово Яманучи 21, тогда как вторая и третья дают слово 12 , которое не является словом Яманучи. Форма одной равна $(2,1)$, другой - $(1,1,1)$. Так что

$$
s_{(1)} s_{(1,1)}=s_{(1,1,1)}+s_{(2,1)} \text {. }
$$

Конечно, в этом легко убедиться и непосредственно, так как $s_{(1)}=x+y+z, s(1,1)=$ $x y+x z+y z, s_{(1,1,1)}=x y z$, a $s_{(2,1)}=x x y+x x z+x y y+2 x y z+y y z+x z z+y z z$.

12.7. Правила Пьери. Предыдущий пример есть частный случай более общей ситуации. Предположим, что $\nu=(1, \ldots, 1)=\left(1^{k}\right)$. Существует единственное слово Яманучи веса $\nu$, а именно слово $\kappa=k^{\prime}(k-1)^{\prime} \ldots 2^{\prime} 1^{\prime}$ (штрихи мы ставим, чтобы подчеркнуть, что это символы алфавита $I^{\prime}$ ). Когда мы хотим расширить диаграмму $\mu$ до диаграммы $\lambda$ так, чтобы в разность $\lambda \backslash \mu$ можно было вписать слово $\kappa$, у нас возникает единственный запрет - нельзя ставить два символа в одну строчку. В самом деле, иначе они строго убывают в строке, что запрещается условием полустандартности. Так что мы получаем в этом случае чисто диаграммный ответ:

$$
s_{\mu} s_{\left(1^{k}\right)}=\sum_{\lambda} s_{\lambda}
$$

где $\lambda$ пробегает множество таких расширений диаграммы $\mu$, что разность $\lambda \backslash \mu$ является “вертикальной полосой” размера $k$.

Похоже вьглядит случай $\nu=(k)$. Снова имеется единственное слово Яманучи такого веса, а именно $\alpha=1^{\prime} \ldots 1^{\prime}=1^{\prime k}$. Когда мы хотим расширить диаграмму $\mu$ до диаграммы $\lambda$ так, чтобы в разность $\lambda \backslash \mu$ можно было вписать слово $\alpha$, у нас возникает единственньй запрет - нельзя ставить два символа в один столбец. Поэтому

$$
s_{\mu} s_{(k)}=\sum_{\lambda} s_{\lambda}
$$

где $\lambda$ пробегает множество таких расширений диаграммы $\mu$, что разность $\lambda \backslash \mu$ является "горизонтальной полосой" размера $k$.

12.8. Иногда правило Литтлвуда-Ричардсона формулируется в терминах, дуальных к теореме 12.5. Для этого транспонируем стандартную пару $(a, b)$, т.е. рассмотрим массив $(a, b)^{T}=\left(\begin{array}{c}b^{T} \\ a^{T}\end{array}\right)$. Условия стандартности 1)-3) (см. п. 8.4) преврашаются тогда в условия:

$\left.1^{\prime}\right)$ массив $\left(\begin{array}{c}b^{T} \\ a^{T}\end{array}\right)$ L-плотньй;

$\left.2^{\prime}\right)$ массив $a^{T}$ биплотньй формы (веса) $\mu$;

$\left.3^{\prime}\right)$ массив $b^{T}$ D-плотный “вертикального” веса $\nu$.

Условие $3^{\prime}$ ) на словесном языке означает, что мы имеем слово-таблицу $t$ веса $\nu$. А условие $1^{\prime}$ ) означает, что если мы приставим к тексту $t$ (единственное) слово-строку Яманучи веса $\mu$ (обозначим это слово снова как $\mu$ ), то получившийся текст $t \cdot \mu$ будет словом Яманучи. Иными словами, слово-таблица $t$ должно стать словом Яманучи после добавления в конец слова Яманучи $\mu$. Такая формулировка приведена в работе [38]. 


\section{3. Соответствие Робинсона-Шенстеда-Кнута}

13.1. Теорема 6.2 о биекции вызьвает живейшие аналогии со знаменитым соответствием Робинсона-Шенстеда-Кнута $(R S K)$. В самом деле, она говорит, что задать массив $a$ - это то же самое, что задать два массива $\mathbf{L} a$ и $\mathbf{D} a$ (которые имеют одну и ту же форму). Соответствие $R S K$ тоже по целочисленному массиву (матрице или тексту-слову $w)$ строит две таблицы $P(w)$ и $Q(w)$ одинаковой формы. И точно так же это соответствие является биекцией. Естественно спросить - как связаны между собой эти две конструкции?

Мы уже объяснили выше, как по слову-тексту $w$ строится текст-таблица $P(w)$ (или $P(a)$ по массиву $a)$. И видели в п. 10.4 , что эта таблица соответствует $\mathbf{D}(a)$, если $w=w(a)$. Однако $Q(a)$ не совпадает с таблищей, соответствуюшей $\mathbf{D}$-плотному массиву $\mathbf{L}(a)^{T}$. Это видно на простейших примерах. Причина такого несовпадения тоже достаточно проста, и мы скажем об этом в следуюшем пункте.

13.2. Мы не будем давать точного определения таблищы $Q(w)$. Вместо этого мы воспользуемся фактом (установленным Д. Кнутом [34]; см. также [21]), что $Q(a)$ - это фактически $P$, но примененное не к матрице $a$, а к транспонированной матрище. Чтобы не путаться с операцией транспонирования массива из п. 2.3, обозначим применение этой операции к массиву $a$ как $a^{M}$. Предположим, что $a$ имеет конечньй формат $I \times J$. Тогда $a^{M}-$ массив формата $J \times I$ и

$$
a^{M}(j, i)=a(n+1-i, m+1-j) .
$$

Так вот, $Q$-символ $Q(a)=P\left(a^{M}\right)$. Заметим, что это текст-таблица в алфавите $J$. Конечно, мы можем понимать $Q(a)$ как $\mathbf{D}$-плотньй массив формата $J \times I$.

Основная формула, связьвающая $R S K$ и нашу биекцию, задается как

$$
Q(a)=\mathbf{L}(* a)^{T}=\mathbf{D}\left(* a^{T}\right) .
$$

В самом деле, $Q(a)=P\left(a^{M}\right)=\mathbf{D}\left(a^{M}\right)=\mathbf{L}\left(a^{M T}\right)^{T}$. Остается заметить, что $a^{M T}=* a$.

Можно также сказать, что $Q$-таблица Кнута $Q(a)$ получается из нашего $\mathbf{D}$-плотного массива $\mathbf{L}(a)^{T}$ применением инволюции Шютценберже. В самом деле, инволюция Шютценберже, примененная к массиву $\mathbf{L}(a)^{T}=\mathbf{D}\left(a^{T}\right)$, по определению равна

$$
\mathbf{D}\left(* \mathbf{D}\left(a^{T}\right)\right)=\mathbf{D}\left(\mathbf{U}\left(* a^{T}\right)\right)=\mathbf{D}\left(* a^{T}\right) .
$$

13.3. Приведем несколько классических применений соответствия $R S K$ или нашей биекции 6.2. Напомним, что произвольному массиву а мы сопоставляли два массива специального вида: лево-плотньй массив $\mathbf{L} a$ и нижне-плотньй массив $\mathbf{D} a$, причем формы $\mathbf{L} a$ и $\mathbf{D} a$ были одни и те же. И это биекция. Отметим также, что $J$-вес $\mathbf{L} a$ совпадает с $J$-весом $a$, а $I$-вес $\mathbf{D} a$ равен $I$-весу $a$.

а) Предположим, что (целочисленный) массив $a$ обладает свойством, что каждый его столбец содержит единственный шар. Тогда и его уплотнение $\mathbf{D} a$ обладает тем же свойством. Если мы будем читать $\mathbf{D} a$ как текст-таблицу, то каждая буква будет встречаться ровно один раз. Такие таблицы назьваются стандартнымми.

Массив $a$ с таким свойством можно понимать как отображение $a: I \rightarrow J . R S K$ заставляет нас непривычно смотреть на это отображение. При стандартном подходе мы 
интересовались бы в первую очередь тем, сколько прообразов у каждого элемента $j \in J$. То есть интересовались бы $J$-весом $a$. Теперь для нас главная характеристика - форма $a$, т.е. разбиение $\mathbf{L} \mathbf{D} a$. Нижний конец $\mathbf{D} a$ - это тоже связанное с $a$ отображение из $I$ в $J$ какого-то специального вида.

b) Симметрично для строк. В этом случае $\mathbf{L} a$ в каждой строке содержит единственный шар. И соответственно $I$-слово-таблица $w\left(\mathbf{L} a^{T}\right)$ будет стандартной.

c) Представим теперь, что вьполнены оба условия. Это означает, что массив $a$, рассмотренньй как отображение из $I$ в $J$, является биекцией. (То есть если отождествить $I$ и $J$ с сохранением порядка, то мы имеем перестановку $I$.) Мы видим, что каждая перестановка множества $I$ из $n$ элементов кодируется двумя стандартными таблицами одной и той же формы. Замена перестановки на обратную соответствует транспозищии соответствуюшего массива, а значит, инверсии этих двух таблиц. Именно это обнаружил К. Шенстед [46].

d) Представим теперь, что массив $a$ симметричен, $a=a^{T}$. Тогда очевидно, что $(\mathbf{L} a)^{t}=\mathbf{D} a^{t}=\mathbf{D} a$, т.е. что обе таблицы, ассоциированные с $a$, совпадают. Мы получаем, что задать симметричньй массив формата $I \times I-$ то же самое, что задать одну таблищу в алфавите $I$. Или, что правильнее, один $\mathbf{D}$-плотньй массив $d$. Или, как говорилось в п. 8.5, паттерн Гельфанда-Цетлина.

е) Наконец, если $a$ - симметричньй и биективньй массив (т.е. представляет инволютивную перестановку множества из $n$ элементов), то он однозначно задается произвольной стандартной таблицей размера $n$.

13.4. ЗАмЕчАНИЕ. Интересно было бы сравнить конструкцию d) с другой, которую можно назвать конструкцией Рэлея. Будем понимать симметричньй массив $a$ как симметрическую матрицу. Обозначим через $\lambda=\lambda_{n}$ ее спектр. Затем отрежем от матрицы $a n$-е строчку и столбец и с полученной $(n-1)$-матрицей проделаем то же самое, получив новую убьвающую последовательность-спектр $\lambda_{n-1}$. Главньй факт состоит в том, что $n$-вектор $\lambda_{n}$ и $(n-1)$-вектор $\lambda_{n-1}$ перемежаются (теорема Коши-Рэлея). Продолжая урезать нашу матрицу, мы получим последовательность

$$
\lambda_{n} \supset \cdots \supset \lambda_{1}
$$

соседние члены которой перемежаются. То есть получим некоторьй паттерн Гельфанда-Цетлина (треугольник Рэлея).

Конечно, это разные паттерны. Например, собственные значения могут быть отрицательными, тогда как форма массива всегда $\geqslant 0$. Если массив $a$ целочисленньй, форма тоже целочисленная, тогда как спектр может не быть целым. Наконец, массив $\mathbf{D} a$ однозначно определяет симметричный массив $a$, тогда как отображение Рэлея (из симметрических матриц в паттерны), хотя и сюръективно (теорема Хорна), но не инъективно, а только конечно.

13.5. Обычно на факты с) и е) смотрят обезличенно, т.е. как на соотношения между числами. Число стандартных таблиц формы $\lambda$ обозначается $f_{\lambda}$. И тогда утверждение пункта с) преврашается в тождество Фробениуса

$$
n !=\sum_{\lambda} f_{\lambda}^{2}
$$

а утверждение пункта е) - в утверждение, что в симметрической группе $S_{n}$ имеется $\sum_{\lambda} f_{\lambda}$ инволюций. 
Для числа $f_{\lambda}$ (т.е. для числа Костки $\left.K_{\lambda,(1, \ldots, 1}\right)$ имеется симпатичная формула крюков. Много других фактов можно найти в книгах [21], [40], [45], [48].

На факты типа d) тоже можно смотреть обезличенно, как на числа. Однако так как тут участвует явно бесконечное число объектов, нужно их снабдить ярлыками и считать по отдельности.

13.6. Формула Коши. Начнем с общей биекции 6.2 (или модифицированного $R S K)$. Пусть сначала у нас имеется один шар, расположенньй в клетке $(i, j)$. Его бивесом назовем выражение $x_{i} y_{j}$. Пусть $a$-целочисленньй массив. Переберем все его шары и перемножим их веса. То есть мы сопоставляем массиву $а$ моном $x^{a} y^{a^{t}}$ в обозначениях п. 11.1 .

Так как операция $\mathbf{L}$ сохраняет $J$-вес, $y^{a^{t}}$ является мономом таблицы $\mathbf{L} a$. Симметрично, $x^{a}$ является мономом таблицы $\mathbf{D} a$. Таким образом, $x^{a} y^{a^{t}}=y^{\mathbf{L} a} x^{\mathbf{D} a}$, и сумма мономов $x^{a} y^{a^{t}}$ по всем массивам $a$ фиксированной формы $\lambda=\mathbf{L D} a$ есть произведение функций Шура $s_{\lambda}(x)$ и $s_{\lambda}(y)$. Соответственно, если мы просуммируем по всем формам, т.е. фактически по всем массивам из $\mathscr{A}_{\mathbb{Z}}(I \times J)$, мы получим сумму $\sum_{\lambda} s_{\lambda}(x) s_{\lambda}(y)$.

Теперь посчитаем эту сумму по-другому. А именно, рассмотрим произведение $\prod_{i, j}\left(1+x_{i} y_{j}+x_{i}^{2} y_{j}^{2}+\cdots\right)=\prod_{i, j}\left(1-x_{i} y_{j}\right)^{-1}$. Каждый моном этого произведения соответствует в точности некоторому массиву (если в массиве $a$ на $(i, j)$-м месте находилось $a(i, j)$ шаров, то надо из сомножителя с номером $i j$ взять $\left.\left(x_{i} y_{j}\right)^{a(i, j)}\right)$. Так что это произведение есть снова сумма мономов по всем массивам $a$. В общем, мы получаем формулу Коши

$$
\prod_{i, j}\left(1-x_{i} y_{j}\right)^{-1}=\sum_{\lambda} s_{\lambda}\left(x_{1}, \ldots, x_{n}\right) s_{\lambda}\left(y_{1}, \ldots, y_{m}\right)
$$

13.7. Тождество Шура. Аналогично можно посмотреть на сумму по всем симметричньм массивам. Теперь с симметричным массивом $a$ свяжем моном $x^{a}=\sqrt{x^{a} x^{a^{t}}}$. Сумма этих мономов по всем симметричньм массивам формы $\lambda$ приводит тогда к функции Шура $s_{\lambda}(x)$. А сумма по всем симметричным массивам дает $\sum_{\lambda} s_{\lambda}(x)$. С другой стороны, эту сумму можно представить как произведение

$$
\prod_{i}\left(1+x_{i}+x_{i}^{2}+\cdots\right) \prod_{i<j}\left(1+x_{i} x_{j}+x_{i}^{2} x_{j}^{2}+\cdots\right)=\prod_{i}\left(1-x_{i}\right)^{-1} \prod_{i<j}\left(1-x_{i} x_{j}\right)^{-1} .
$$

Это так назьваемое тождество Шура.

\section{4. Дуальные таблицы и плоские разбиения}

14.1. Дуальные таблищы. Пусть $T$ - полустандартная таблица формы $\lambda$, заполненная символами из алфавита $I=\{1, \ldots, n\}$. Построим по ней дуальную (или дополнительную) таблицу $T^{*}$. Делается это так (см. [50]).

Рассмотрим столбец таблищы $T$. Написанные в этом столбце символы будем понимать как элементы множества $I$; так как все эти символы различны, мы можем понимать столбец как подмножество в $I$. Таким образом, таблища $T$ дает нам семейство подмножеств $T_{1}, \ldots, T_{l} \subset I$, где $l=\lambda(1)$. Это семейство не произвольное: оно монотонное относительно одного интересного частичного порядка $\preceq$ на $2^{I}$, которое мы назовем доминированием (оно рассматривалось в [50]). 
А именно, пусть даны два подмножества: $A=\left\{a_{1}, \ldots, a_{s}\right\}$ и $B=\left\{b_{1}, \ldots, b_{t}\right\}$. Скажем, что $B$ доминирует $A(A \preceq B)$, если $t \leqslant s$ и $a_{k} \leqslant b_{k}$ для любого $k \leqslant t$. Наглядно множество $B$ расположено в $I$ "вьше", чем $A$. (Можно показать, что посет $\left(2^{I}, \preceq\right)$ является дистрибутивной решеткой.) Очевидно, что условие полустандартности (точнее, что символы нестрого возрастают при движении вправо) в точности совпадает с условием монотонности $T_{1} \preceq \cdots \preceq T_{l}$. И мы получаем достаточно тривиальное утверждение: задать полустандартную таблииу - то жее самое, что задать монотонный путь длины $\lambda(1)$ в $2^{I}$.

Полезно немного переписать условие доминирования. А именно, для подмножества $A \subset I$ обозначим через $\int A$ функцию на $I$, которая точке $i \in I$ сопоставляет число элементов в множестве $A \cap\{1, \ldots, i\}$. Имеет место следуюший очевидный факт.

14.2. Лемма. $A \preceq B$ тогда и только тогда, когда $\int A \geqslant \int B$.

СледСтвие. $Е с л и \quad A \preceq B, m o I \backslash B \preceq I \backslash A$.

В самом деле, $\left(\int(I \backslash A)\right)(i)=i-\left(\int A\right)(i)$.

14.3. Приступим теперь к построению дуальной таблишы. Пусть $T$ - полустандартная таблица, заданная как монотонная последовательность подмножеств $I: T_{1} \preceq$ $\cdots \preceq T_{l}$. Рассмотрим дополнительные множества $I \backslash T_{k}, 1 \leqslant k \leqslant l$. Тогда монотонной является последовательность $I \backslash T_{l} \preceq \cdots \preceq I \backslash T_{1}$, и она-то и задает дуальную таблицу.

ПримеР. Пусть таблица $T$ имеет вид

$$
T=\begin{array}{llll}
4 & & & \\
3 & 4 & & \\
1 & 2 & 2 & 3
\end{array}
$$

и $n=4$. Тогда дуальная таблица $T^{*}$ имеет вид

$$
T^{*}=\begin{array}{llll}
4 & 4 & & \\
2 & 3 & 3 & \\
1 & 1 & 1 & 2
\end{array}
$$

Для дальнейших целей удобно нарисовать эту таблицу, отразив ее от центра. Так, таблица из нашего примера будет вьглядеть как

$$
\begin{array}{llll}
2 & 1 & 1 & 1 \\
& 3 & 3 & 2 \\
& & 4 & 4
\end{array} .
$$

Теперь по строчкам символы идут в убывающем порядке, а по столбцам - в строго убьвающем. Чтобы сохранить привычный паттерн монотонности, нужно сменить порядок в $I$ на противоположный $I^{*}=\left\{n^{*}>\cdots>1^{*}\right\}$. И мы получим косую полустандартную таблицу

$$
\begin{array}{llll}
2^{*} & 1^{*} & 1^{*} & 1^{*} \\
& 3^{*} & 3^{*} & 2^{*} \\
& & 4^{*} & 4^{*}
\end{array}
$$

которую снова обозначим $T^{*}$. 
Теперь решающее место. Предположим, что у нас есть две полустандартные таблищы одной и той же формы $\lambda: T$ с алфавитом $I$ и $T^{\prime}$ с алфавитом $J$. Совмешая таблищу $T^{\prime}$ с перевернутой таблицей $T^{*}$, мы получаем таблицу прямоугольной формы (с $n$ строчками и $l=\lambda(1)$ столбцами), заполненную символами из алфавита $J \sqcup I^{*}=$ $\left\{1<\cdots<m<n^{*}<\cdots<1^{*}\right\}$. Например, если $T$ как вьше в примере, а

$$
T^{\prime}=\begin{array}{llll}
4 & & & \\
2 & 3 & & \\
1 & 1 & 3 & 4
\end{array},
$$

мы получаем прямоугольную таблицу

$$
\begin{array}{llll}
2^{*} & 1^{*} & 1^{*} & 1^{*} \\
4 & 3^{*} & 3^{*} & 2^{*} \\
2 & 3 & 4^{*} & 4^{*} \\
1 & 1 & 3 & 4
\end{array}
$$

Видно, что эта таблица полустандартная. И это верно в общем случае. Строгое возрастание по столбцам тривиально. Возрастание по строкам внутри $T$ есть по определению, внутри $T^{*}$ - доказано вьше. А если мы внутри строки переходим из $T$ в $T^{*}$, мы имеем даже строгое возрастание.

Очевидно, что эта конструкция обратима. Пусть у нас имеется прямоугольная размера $n \times l$ полустандартная таблища, заполненная алфавитом $J \sqcup I^{*}$. Тогда ее можно разделить на "юго-западную" часть, заполненную $J$, и “северо-восточную”, заполненную $I^{*}$. Первая часть дает таблицу $T^{\prime}$, а дуализация второй части - таблицу $T$. Формы $\lambda$ обеих таблиц одинаковы (при этом $\lambda(1) \leqslant l$ ).

Итак мы получили следуюшее предложение.

14.4. ПРЕДЛОЖЕНИЕ. Приведенная конструкция устанавливает биекцию между множсеством пар $\left(T, T^{\prime}\right)$ полустандартных таблич одной формы ( с числом столбцов $\leqslant l$, таблица $T$ заполнена из алфавита $I$, таблица $T^{\prime}$ из $J)$ и множеством полустандартных таблич прямоугольной формы $\left(l^{n}\right)$, заполненных алфавитом $J \sqcup I^{*}$.

14.5. Рассуждая как в примере 12.6, мы получаем равенство многочленов Шура

$$
\sum_{\lambda(1) \leqslant l} s_{\lambda}\left(x_{1}, \ldots, x_{n}\right) s_{\lambda}\left(y_{1}, \ldots, y_{m}\right)=\left(x_{1} \cdots x_{n}\right)^{l} s_{\left(l^{n}\right)}\left(x_{1}^{-1}, \ldots, x_{n}^{-1}, y_{1}, \ldots, y_{m}\right) \text {. }
$$

14.6. Плоские разбиения. Напомним, что плоским разбиением (формата $I \times J)$ называется монотонно убьвающая функция $p: I \times J \rightarrow \mathbb{Z}_{+}$. Ясно, что плоские разбиения представляют из себя двумерные (или трехмерные, как посмотреть) обобщения разбиений или диаграмм Юнга. Исследование их было начато П. А. Макмагоном в конце XIX столетия; историю и дальнейшее развитие см. в [48]. Для плоского разбиения $p$ обозначим $|p|=\sum_{i, j} p(i, j)$. След плоского разбиения есть $\operatorname{tr}(p)=\sum_{i} p(i, i)-$ сумма диагональных элементов.

Пусть у нас имеется полустандартная таблица прямоугольной $(n \times l)$ формы с заполнением из алфавита $\{1, \ldots, n+m\}$ (о таких таблищах мы только что говорили). Построим по такой таблище плоское разбиение $p$. Для точки $(i, j) \in I \times J$ положим 
$p(i, j)$ равньм числу столбцов таблицы, у которых на высоте $i$ стоит символ, не превьшаюший $m-j+i$.

Например, для нашей таблицы (у которой 4 * обозначено как 5 и т.п.)

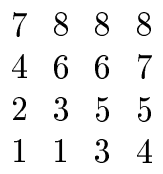

мы получаем плоское разбиение

$\begin{array}{llll}2 & 1 & 0 & 0 \\ 2 & 2 & 1 & 0 \\ 3 & 2 & 1 & 0 \\ 4 & 4 & 3 & 1\end{array}$

В общем случае значение функции $p$ в “начальной” (или минимальной) точке $(1,1)$ равно числу столбцов, которые начинаются с символа $\leqslant m$. (Ясно, что если столбец начинается с символа $>m$, то этот символ равен $m+1$, а весь столбец имеет вид $(m+1, \ldots, m+n)^{T}$.) Обозначим через $\lambda$ форму (поддиаграмму) в нашем прямоугольнике, заполненную символами $\leqslant m$ (т.е. это "юго-западная" часть исходной таблицы). Тогда по определению $p(1,1)=\lambda(1)$.

И вообше, значение нашей функции $p$ в диагональной точке $(i, i)$ равно длине $i$-й строки диаграммы $\lambda$, т.е. равно $\lambda(i)$.

Так же легко найти значение в любой точке выше диагонали, т.е. когда $j \geqslant i$. Для $k \geqslant 0$ обозначим как $\lambda_{k}$ поддиаграмму нашей таблищы, заполненную числами $\leqslant m-k$ (так что $\left.\lambda=\lambda_{0}\right)$. Тогда

$$
p(i, i+k)=\lambda_{k}(i) .
$$

Аналогичное соотношение верно и для точек ниже диагонали. Только тут следует помнить, что нужно взять "северо-восточную часть" нашей таблишы, потом ее дуализировать и уже для этой полустандартной таблицы формы $\lambda$ брать фильтрацию Юнга.

Отсюда видно, что функция $p$ нестрого убьвает по $i$ и $j$. Например, вьше диагонали монотонность по $j$ очевидна, поскольку $\lambda_{k} \supset \lambda_{k+1}$. Убывание по $i$ - это следствие более тонкой перемежаемости (см. пш. 8.4-8.5). Так что мы получаем, что построенная функция $p$ монотонно убывает, т.е. является плоским разбиением.

Конечно, эта конструкция обратима. Если у нас имеется плоское разбиение $p$, мы без труда можем образовать две полустандартных таблищы формы $\lambda$ (где $\lambda(j)=p(j, j)$ для $j \leqslant \min (n, m))$. Одна таблица $T^{\prime}$ (с заполнением $J$ ) строится по значениям $p$ вьше диагонали, другая $T$ (с заполнением $I$ ) - по значениям ниже диагонали. Тем самым мы получаем следующее предложение.

14.7. ПРЕДЛОЖЕНИЕ. Множество плоских разбиений формата $I \times J$ биективно соответствует (указанным выше способом) множсеству пар полустандартных таблии одной и той же формы $\lambda$, причем $\lambda(1) \leqslant p(1,1)$.

А теперь самое время вспомнить про нашу биекцию 6.2. Мы по (целочисленному) массиву $а$ строили две полустандартные таблицы одинаковой формы: $T$ - соответствующую $\mathbf{D} a$, и $T^{\prime}$ - соответствующую $\mathbf{L} a^{T}$. Отсюда мы получаем следующее утверждение. 
14.8. ТЕОРемА. Приведенное выше соответствие устанавливает биекцию между множеством иельх массивов формата $I \times J$ и множсеством плоских разбиений того же формата. ${ }^{3}$

14.9. Если $a-$ массив, а $p=p(a)$ - соответствуюшее плоское разбиение, то можно еще кое-что сказать о связи между ними. Мы уже отмечали, что максимум функции $p$, равный $p(1,1)$, равен $\lambda(1)$, где $\lambda=\Phi(a)$ - форма нашего массива. (В дополнении В мы проинтерпретируем $\lambda(1)$ как величину максимальной антицепи массива $a$.) Проще сказать, чему равна сумма значений $p$ на диагонали; это $|\lambda|$, т.е. тотальный вес массива $a$ :

$$
p(1,1)+p(2,2)+\cdots+p(k, k)=\sum_{i, j} a(i, j)
$$

$(k=\min (n, m))$. Аналогично вычисляется сумма $p(1,2)+p(2,3)+\cdots$. Она равна $\left|\lambda_{1}\right|$, т.е. весу массива $а$ без верхней $(m-$ й $)$ строки. И так далее, для $k \geqslant 0$

$$
p(1,1+k)+p(2,2+k)+\cdots=\sum_{i, 1 \leqslant j \leqslant m-k} a(i, j) .
$$

Аналогично находятся суммы при отрицательном $k$. В любом случае мы получаем выражение для суммы всех $p(i, j)$ :

$$
|p(a)|:=\sum_{i, j} p(i, j)=\sum_{i, j}(n+m+1-i-j) a(i, j) .
$$

14.10. Имея биекцию между массивами и плоскими разбиениями, можно посчитать число последних. Вернемся к формуле

$$
\prod_{1 \leqslant i \leqslant n, 1 \leqslant j \leqslant m} \frac{1}{1-x_{i} y_{j}}=\sum_{a} x^{a} y^{a^{t}}
$$

из п. 13.6, где суммирование идет по всем массивам $a$.

Сделаем подстановку $x_{i}=q t^{n-i+1}, y_{j}=t^{m-j}$. Каждьй сомножитель $\left(x_{i} y_{j}\right)^{a(i, j)}$ превратится в $q^{a(i, j)} t^{(n+m-i-j+1) a(i, j)}$; соответственно каждьй моном $x^{a} y^{a^{t}}$ превратится в моном $q^{\sum_{i, j} a(i, j)} t^{\sum_{i, j}(n+m-i-j+1) a(i, j)}$. Интерпретируя $\sum_{i, j} a(i, j)$ как след соответствуюшего плоского разбиения $p(a)$, а $\sum_{i, j}(n+m-i-j+1) a(i, j)-$ как $|p(a)|$, мы можем переписать этот моном как $q^{\operatorname{tr}(p(a))} t^{|p(a)|}$. А пользуясь биекцией между массивами и плоскими разбиениями, переписьваем правую сумму в $(* *)$ как сумму $\sum_{p} q^{\operatorname{tr}(p)} t^{|p|}$ по всем плоским разбиениям $p$ формата $I \times J$.

Левая часть тождества переписывается как произведение выражений

$$
\frac{1}{1-q t^{n+m-i-j+1}}
$$

где $i$ пробегает от 1 до $n$, а $j$ - от 1 до $m$. Но это то же самое, что произведение выражений

$$
\frac{1}{1-q t^{i+j-1}}
$$

\footnotetext{
${ }^{3}$ Из $(*)$ и биекции 6.2 следует, что эта теорема верна и без требования целочисленности массивов и плоских разбиений.
} 
где $i$ и $j$ меняются в тех же пределах. И мы получаем знаменитую формулу Стенли, см. [48]:

$$
\sum_{p} q^{\operatorname{tr}(p)} t^{|p|}=\prod_{i=1}^{n} \prod_{j=1}^{m}\left(1-q t^{i+j-1}\right)^{-1}
$$

В частности, при $q=1$ получаем

$$
\sum_{p} t^{|p|}=\prod_{i=1}^{n} \prod_{j=1}^{m} \frac{1}{1-t^{i+j-1}}
$$

Пусть, для определенности, $n \leqslant m$. Тогда правая сторона есть произведение

$$
\frac{1}{\left[(1-t)\left(1-t^{2}\right)^{2} \cdots\left(1-t^{n}\right)^{n}\right] \cdots\left[\left(1-t^{m}\right)^{n}\left(1-t^{m+1}\right)^{n-1} \cdots\left(1-t^{n+m-1}\right)\right]} .
$$

Если устремить $m$ и $n$ к бесконечности, мы получим еще более компактную формулу Макмагона

$$
\sum_{p} t^{|p|}=\prod_{k}\left(1-t^{k}\right)^{-k}
$$

где слева суммирование идет уже по всем плоским разбиениям. Полезно сравнить ее с формулой Эйлера для обычных разбиений:

$$
\sum_{\lambda} t^{|\lambda|}=\prod_{k}\left(1-t^{k}\right)^{-1}
$$

14.11. Можно проделать то же самое, зафиксировав форму $\lambda$ массива $a$ (или ограничение на диагональ $j=i$ плоского разбиения $p$ ). Сумма $\sum_{a} x^{a} y^{a^{t}}$ по таким массивам равна, в силу $R S K, s_{\lambda}(x) s_{\lambda}(y)$. Если мы теперь просуммируем по всем $\lambda$ с $\lambda(1) \leqslant l$ и воспользуемся равенством п. 14.5, мы получим (после той же подстановки $\left.x_{i}=q t^{n-i+1}, y_{j}=t^{m-j}\right)$, что

$$
\sum_{p, p(1,1) \leqslant l} q^{\operatorname{tr}(p)} t^{|p|}=t^{l m(m-1) / 2} s_{\left(l^{m}\right)}\left(q t^{n}, \ldots, q t, 1, t^{-1}, \ldots, t^{-m+1}\right)
$$

Снова стоит положить $q=1$; получаем формулу для числа трехмерных диаграмм Юнга, вписанных в параллелепипед размера $n \times m \times l$ :

$$
\begin{aligned}
\sum_{p, p(1,1) \leqslant l} t^{|p|} & =t^{l m(m-1) / 2} s_{\left(l^{m}\right)}\left(t^{n}, \ldots, t, 1, t^{-1}, \ldots, t^{-m+1}\right) \\
& =t^{-l m(m-1) / 2} s_{\left(l^{m}\right)}\left(t^{n+m-1}, \ldots, t^{m}, t^{m-1}, \ldots, 1\right),
\end{aligned}
$$

что совпадает с формулой (7.108) из [48]. 


\section{Дополнение А. Действие симметрической группы}

A.1. Пользуясь операциями $D$ и $U$, можно ввести на множестве массивов действие группы $S(J)$ перестановок "вертикального" алфавита $J$. Конечно, симметричным образом можно ввести и действие $\mathscr{S}(I)$. Мы воспроизводим здесь конструкцию Ласку-Шютценберже ([39], см. также [38]).

Сразу действие произвольной перестановки задать почему-то не удается. Поэтому приходится действовать через элементарные транспозищии $\sigma_{j}$, переставляюшие соседние буквы $j$ и $j+1$. Хорошо известно, что такие транспозищии $\sigma_{j}, j=1, \ldots, m-1$, порождают группу $S(J)$. Эти образуюшие связаны соотношениями Мура-Кокстера:

a) $\sigma_{j}^{2}=1$

b) $\sigma_{j} \sigma_{k}=\sigma_{k} \sigma_{j}$, если $j$ и $k$ не соседние, и

c) $\sigma_{j} \sigma_{j+1} \sigma_{j}=\sigma_{j+1} \sigma_{j} \sigma_{j+1}$ для любого $j=1, \ldots, m-2$.

Более того, эти соотношения определяют группу $S(J)$.

Поэтому достаточно задать действие только образующих транспозиций $\sigma_{j}$ и проверить вьполнение соотношений Мура-Кокстера. Из приводимого далее определения этого действия видно, что оно согласовано с отображением $J$-веса $w t_{J}: \mathscr{A} \rightarrow \mathbb{R} \otimes J$.

А.2. Определим действие $\sigma_{j}$, которое влияет лишь на строки $j$ и $j+1$. В силу этого можно считать, что мы имеем дело с двухстрочным массивом, содержашим строки 1 (нижняя) и 2 (верхняя). В нижней строке находится $r_{1}$ шаров, в верхней $-r_{2}$ шаров. Рассмотрим сначала случай $r_{2} \geqslant r_{1}$. В этом случае по определению $\sigma=D^{r_{2}-r_{1}}$. Заметим, что в верхней строчке есть по меньшей мере $r_{2}-r_{1}$ свободных шаров. Более точно, пусть $\rho_{2}-$ число свободных шаров в верхней строчке и $\rho_{1}-$ число свободных шаров в нижней. Так как все остальные шары спарены, то $\rho_{2}-\rho_{1}=r_{2}-r_{1}$. Операция $\sigma$ перемешает вниз $r_{2}-r_{1}$ самых правых свободных шаров. После этого число свободных шаров внизу становится равно $\rho_{2}$, а наверху остается $\rho_{1}$.

Рассмотрим теперь противоположньй случай: $r_{1} \geqslant r_{2}$. В этой ситуации по определению $\sigma=U^{r_{1}-r_{2}}$ (если угодно, $\sigma$ снова равно $D^{r_{2}-r_{1}}$ ). Ясно из предыдущего описания, что $\sigma \sigma a=a$.

А.3. Продуктивно представлять себе действие $\sigma$ в терминах некоторой модификации стабильного матчинга. А именно, представим, что верхние шары образовали стабильный матчинг с нижними, как в разделе 3 . После этого самый правьй свободный нижний шар пытается соединиться с самым левым свободным верхним, и т. д. То есть наш массив нужно как бы свернуть в кольцо, заменив цепочку $1 \rightarrow 2 \rightarrow \cdots \rightarrow n$ циклом $1 \rightarrow 2 \rightarrow \cdots \rightarrow n \rightarrow 1$. После такого дополнительного спаривания у нас останутся незанятые шары уже только вверху или только внизу. И операция $\sigma$ просто перемешает их (все) сверху вниз (если они остались наверху) или снизу вверх.

Эту же идею можно выразить с помошю понятия ииклажа (Ласку-Шютценберже). Обозначим через $C$ следующую операцию с массивом: мы берем первый столбец массива и ставим его в конец, т.е. делаем его $(n+1)$-м (получается массив с новьм множеством столбцов $\{2, \ldots, n+1\})$. Довольно легко проверить, что $C$ коммутирует $\mathrm{c} \sigma$.

В общем случае мы определяем действие инволющий $\sigma_{j}$ на строки $j$ и $j+1$. И из предыдушего видна следующая лемма. 
А.4. ЛЕммА. Инволюции $\sigma_{j}$ коммутируют с операцией ииклажа $C$.

А.5. Приступим теперь к проверке соотношений Мура-Кокстера. Про инволютивность $\sigma_{j}$ мы уже говорили. Коммутирование $\sigma_{j}$ и $\sigma_{k}$ для несоседних $j$ и $k$ также очевидно. Поэтому нужно только проверить соотношение

$$
\sigma_{j} \sigma_{j+1} \sigma_{j}=\sigma_{j+1} \sigma_{j} \sigma_{j+1}
$$

применительно к произвольному массиву $a$.

Мы можем считать, что $j=1$, а массив $a$ состоит из трех строк. Далее, так как $D$ и $U$ коммутируют с $L$ и $R$, то инволюции $\sigma_{1}$ и $\sigma_{2}$ коммутируют с $L$ и $R$. Поэтому мы можем считать, что $a-\mathbf{L}$-плотньй массив, имеющий вид

$$
\begin{array}{|l|l|l|}
\hline a & d & f \\
\hline b & e & 0 \\
\hline c & 0 & 0 \\
\hline
\end{array}
$$

Согласно лемме А.4, достаточно проверить это соотношение для массива $C a$, которьй имеет вид

$$
\begin{array}{|l|l|l|}
\hline d & f & a \\
\hline e & 0 & b \\
\hline 0 & 0 & c \\
\hline
\end{array}
$$

L-уплотним его. Точнее, подействуем сначала $\mathbf{L}_{2} ;$ массив примет вид

\begin{tabular}{|c|c|c|}
\hline$d$ & $f+a$ & 0 \\
\hline$e$ & $b$ & 0 \\
\hline 0 & $c$ & 0 \\
\hline
\end{tabular}

Если теперь применить $\mathbf{L}_{1}$, мы получим массив вида

$$
\begin{array}{|l|l|l|}
\hline a^{\prime} & d^{\prime} & 0 \\
\hline b^{\prime} & 0 & 0 \\
\hline c & 0 & 0 \\
\hline
\end{array}
$$

То есть можно считать, что он состоит из двух столбцов, причем $e=0$. Повторяя этот трюк с циклажем и $\mathbf{L}$-уплотнением, мы можем считать, что массив $a$ состоит из единственного столбца. Но теперь выполнение соотношения $\sigma_{1} \sigma_{2} \sigma_{1}=\sigma_{2} \sigma_{1} \sigma_{2}$ уже очевидно.

A.6. ЗАмечАниЕ. Наполустандартных таблицах, а в силу теоремы 6.2 и на произвольных массивах можно определить действие других инволющий $\tau_{i}$, так назьваемых инволюций Бендера-Кнута [3]. Как и инволюции $\sigma_{i}$, они переставляют соседние веса $i$ и $i+1$. Однако эти инволюции не удовлетворяют соотношениям Мура-Кокстера. Фундаментальная роль инволюций Бендера-Кнута в том, что с их помощью выражаются многие другие операции, такие как инволюции $\sigma_{i}$ из п. А.2, инволюция Шютценберже (см. п. 13.2), переключение таблиц (см. п. 11.6). Обстоятельное изучение инволюций Бендера-Кнута предпринято в [33]. 


\section{Дополнение В. Связь с частично упорядоченными множествами}

В этом дополнении мы хотим обсудить еше один подход к соответствию $R S K$, связанньй с представлением массивов как частично упорядоченных множеств (посетов).

В.1. Скажем кратко про историю вопроса. Довольно легко показать, что в любой бесконечной последовательности различных чисел сушествует бесконечная возрастающая или убывающая подпоследовательность. Это простейшее следствие теории Рамсея. В 1935 г. П. Эрдёш и Э. Секереш установили конечньй вариант такого утверждения: в последовательности из $N$ различных чисел существует монотонная подпоследовательность длины $\geqslant \sqrt{N}$; дальнейшее развитие этого результата можно почитать в [17]. В 1961 г. К. Шенстед [46] придумал рецепт нахождения максимальной монотонной подпоследовательности для конкретной перестановки. А именно, с помошью алгоритма вставки строились две (стандартные) таблищы одной формы $\lambda$; фактически это и есть $R S K$. Отметим, что длина максимальной убьвающей подпоследовательности равна $\lambda(1)$. Д. Кнут [34] обобщил этот алгоритм на слова с повторяюшимися буквами. В [25] К. Грин показал, как можно связать диаграмму Юнга (или разбиение $\lambda$, форму посета) с любым конечньм посетом; ширина этой диаграммы $\lambda(1)$ есть максимальная мошность антицепи в посете.

В.2. Для произвольного целочисленного массива $a$ естественным образом определяется посет $\mathscr{P}(a)$. Как множество, посет $\mathscr{P}(a)$ состоит из всех шаров нашего массива $a$. Шар, лежаший в яшике с номером $(i, j)$, считается строго больше шара, лежащего в ящике с номером $\left(i^{\prime}, j^{\prime}\right)$, если $i>i^{\prime}, j>j^{\prime}$. Например, массив

$$
\begin{array}{lllll}
0 & 0 & 0 & 1 & 0 \\
0 & 1 & 0 & 0 & 3 \\
0 & 0 & 0 & 1 & 0 \\
2 & 0 & 0 & 0 & 0 \\
0 & 1 & 0 & 0 & 0
\end{array}
$$

изображается посетом на рис. 10 (как обычно, мы рисуем только "непосредственные" стрелки).

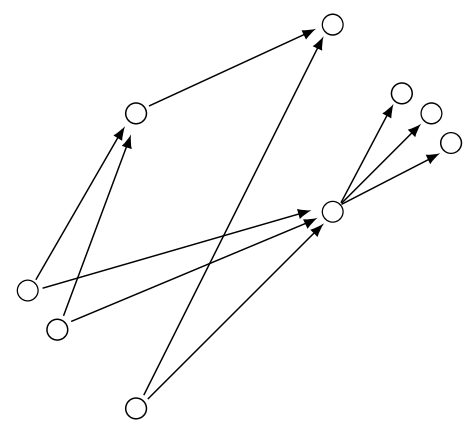

Рис. 10. 
Мы покажем, что для любого целочисленного массива $а$ форма связанного с ним посета $\mathscr{P}(a)$ совпадает с формой массива $a$.

B.3. Для определения формы посета напомним некоторые простые понятия из теории упорядоченных множеств. Цепью посета $\mathscr{P}$ называется подмножество $C \subset \mathscr{P}(a)$, все элементы которого сравнимы между собой. Напротив, антицепью посета назьвается подмножество $A$, в котором все элементы несравнимы. Максимальная длина цепи отражает в каком-то смысле “высоту" посета, тогда как максимальная мошность антицепи - его "ширину".

Для целого $k \geqslant 0$ обозначим $\alpha(k, \mathscr{P})$ (или просто $\alpha(k)$, если понятно, о каком посете $\mathscr{P}$ идет речь) максимальное число элементов в подмножестве, которое можно покрыть $k$ антицепями. Такие подмножества для краткости назьваются $k$-антищепями. Иначе говоря, это подмножества в $\mathscr{P}$ высоты $\leqslant k$. Очевидно, что

$$
0=\alpha(0) \leqslant \alpha(1) \leqslant \cdots .
$$

Оказывается, однако, что эта последовательность $(\alpha(k), k=0,1,2, \ldots)$ не только возрастающая, но и в некотором смысле вогнутая. Более точно, определим (для $k \geqslant 1)$

$$
\varphi(k)=\varphi(k, \mathscr{P})=\alpha(k)-\alpha(k-1) .
$$

К. Грин [25] (а затем С. В. Фомин [18]) доказал, что эта последовательность невозрастающая, т.е. образует разбиение. Вот это разбиение мы и называем формой посета $\mathscr{P}$. Подробнее об этом можно почитать в [7]. Заметим только, что наши обозначения отличаются от обозначений [7]; в частности, у нас "основание" формы $\varphi(1)=\alpha(1)$ соответствует ширине посета, тогда как высота формы (т.е. число ненулевых $\varphi(k))-$ высоте посета. Отметим еще, что сумма всех $\varphi(k)$, т.е. $\alpha(\infty)$, есть в точности мощность посета. Так что форма $\varphi$ действительно дает грубое визуальное представление о посете. Впрочем, разные посеты могут иметь одну и ту же форму.

ЗАмечАниЕ. Можно аналогичную веш проделать с цепями; мы получим тогда сопряженную (или транспонированную) форму.

B.4. ПРимеР [25]. Рассмотрим массив, изображенный на рис. 11, который проще всего ассоциировать со словом $6 \cdot 1 \cdot 7 \cdot 2 \cdot 5 \cdot 8 \cdot 3 \cdot 9 \cdot 4$ или с соответствующей перестановкой девяти элементов. Здесь имеется единственная антицепь размера 5 , ассоциированная с (возрастающей) подпоследовательностью 12589. Поэтому $\varphi(1)=5$. И единственная 2-антищепь размера 8 - это дополнение к шару с номером 5. (Пикантность ситуации в том, что максимальная 2-антищепь не продолжает максимальную антищепь.) Так что форма этого посета равна $(5,3,1)$.

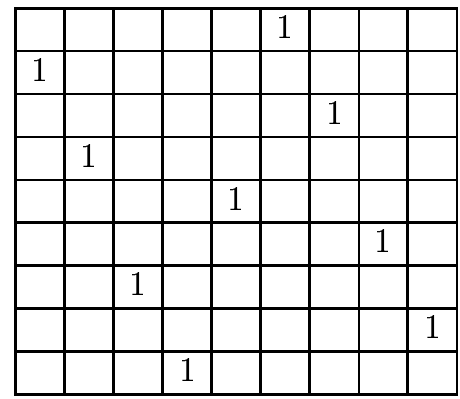

Рис. 11 
Можно непосредственно проверить, что $\mathbf{D} a$ имеет вид

$$
\begin{array}{lllllllll}
0 & 0 & 0 & 0 & 0 & 1 & 0 & 0 & 0 \\
0 & 0 & 0 & 0 & 1 & 0 & 1 & 1 & 0 \\
1 & 1 & 1 & 1 & 0 & 0 & 0 & 0 & 1
\end{array} .
$$

Поэтому форма нашего массива тоже равна $(5,3,1)$. Конечно это не случайно, и верна следующая общая теорема.

B.5. ТЕОремА. Для любого массива а имеем $\Phi(a)=\varphi(\mathscr{P}(a))$.

B.6. Доказательство разбивается на две части. Простая часть состоит в проверке требуемого равенства для биплотного массива, и она совершенно очевидна. В самом деле, в случае биплотного массива $p$ максимальная антицеп состоит из шаров в ящике $(1,1)$. И вообще, $k$-антицепь располагается в $k$ ящиках, и поэтому максимальньй ее размер есть в точности $p(1,1)+\cdots+p(k, k)$.

Основная часть доказательства состоит в проверке инвариантности гриновской формы $\varphi$ относительно операций $D_{j}$ (а также операций $U, L$ и $R$, что уже делается аналогично). Иначе говоря, мы утверждаем, что

$$
\varphi(\mathscr{P}(a))=\varphi\left(\mathscr{P}\left(D_{j} a\right)\right)
$$

для любого $j$ (и аналогично для $U_{j}, L_{i}$ и $R_{i}$ ). Если мы это проверим, мы получим, что $\varphi(\mathscr{P}(a))=\varphi(\mathscr{P}(\mathbf{D} a))=\varphi(\mathscr{P}(\mathbf{L} \mathbf{D} a))$. А последнее, как уже говорилось, и есть $\Phi(a)$.

В.7. ЛЕммА. Для любого $k \geqslant 1$ выполнены неравенства

$$
\varphi(\mathscr{P}(a) ; k) \leqslant \varphi\left(\mathscr{P}\left(D_{j} a\right) ; k\right) \quad u \quad \varphi(\mathscr{P}(a) ; k) \leqslant \varphi\left(\mathscr{P}\left(U_{j} a\right) ; k\right) .
$$

В силу взаимной обратности $U_{j}$ и $D_{j}$ отсюда получается нужное равенство. Более того, мы будем доказьвать только первое неравенство, потому что второе получается аналогично. Чтобы сделать аргументацию более понятной, мы рассмотрим сначала случай $k=1$, т.е. поведение антицепей при действии операций $D$. Если это рассуждение будет понято, обший случай понять уже просто.

B.8. Случай $k=1$. Так как нас интересуют антищепи максимальной мощности (имеется в виду число шаров в антицепи), мы вместе с каждым шаром можем включать и все остальные шары, расположенные в том же ящике. Поэтому антицеп мы будем представлять как множество $A$ яшиков. Единственное требование на $A$ состоит в том, чтобы яшики, входяшие в $A$, не лежали правее и выше один относительно другого. Опять-таки, так как нас интересуют максимальные антицепи, мы можем предполагать, что каждая такая антицеп $A$ устроена как лента; см. рис. 12 .

Итак, пусть даны массив $а$ и лента-антищепь $A$; вес $A$ равен $w(A)=a(A)=\sum a_{(i, j)}$, где сумма берется по ящикам $(i, j) \in A$. Мы действуем на массив операцией $D=D_{j}$, преврашая его в массив $a^{\prime}=D a$. И мы покажем, что существует лента-антицепь $A^{\prime}$ такая, что вес $a^{\prime}\left(A^{\prime}\right) \geqslant a(A)$.

Действие $D=D_{j}$ состоит в том, что некоторьй шар $b$ (расположенньй на уровне $j+1)$ перемешается вертикально вниз на уровень $j$. Если шар $b$ лежал вне $A$, то все очевидно: в качестве $A^{\prime}$ мы можем взять $A$. Поэтому будем считать, что шар лежит в некотором яшике, принадлежашем ленте $A$. Возможны два случая: тривиальньй и нетривиальный. 


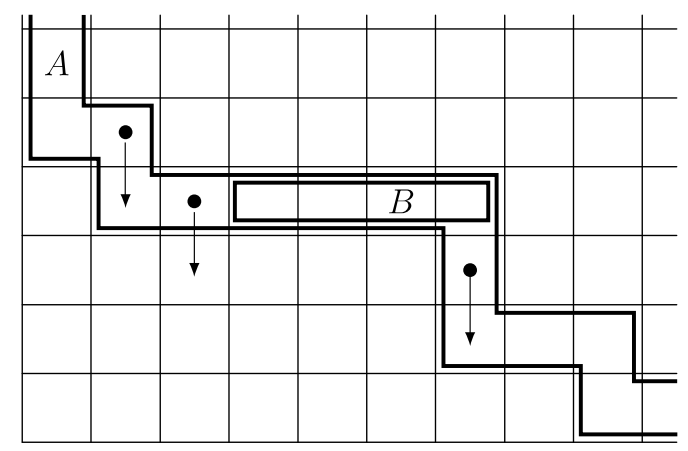

Рис. 12.

Первый, тривиальный, случай состоит в том, что после перемещения шар $b$ снова попадает в яшик из $A$. Например, если он лежал в ячейке $(2,5)$ или $(7,3)$. Тогда в качестве $A^{\prime}$ можно взять $A$.

Второй, нетривиальньй, случай бывает тогда, когда шар $b$ лежал на "горизонтальном" куске ленты $A$, например, в клетке $(3,4)$. И вправо от этой клетки были еще клетки $(4,4),(5,4),(6,4)$ и $(7,4)$ (мы объединяем эти клетки в “брусок" $B)$. В этом случае мы меняем ленту $A$, перемешая брусок $B$ на вектор $(-1,-1)$. Новая антицепь-лента $A^{\prime}$ вьглядит теперь как на рис. 13.

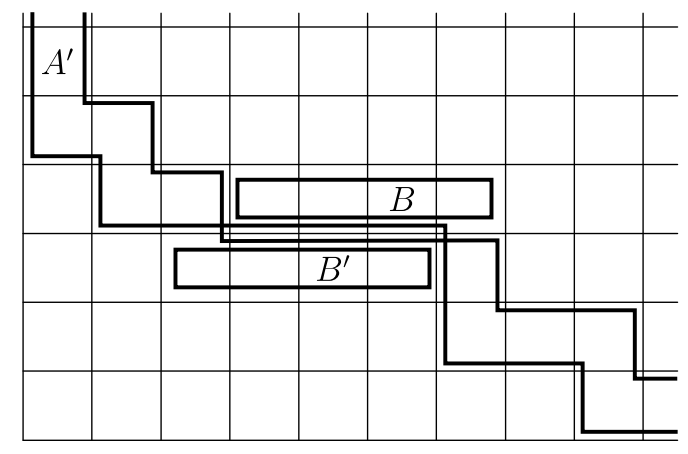

Рис. 13.

Основное утверждение состоит в том, что $a\left(A^{\prime}\right) \geqslant a(A)$. Если мы это установим, то мы в исходной задаче заменим антицепь $A$ на $A^{\prime}$. И тогда мы попадаем в первый, тривиальный случай.

Осталось доказать основное утверждение. Это тоже просто. Так как перемешаемый шар $b$ был самым правым свободным шаром (в стабильном матчинге между уровнями $j+1$ и $j$ ), то любой шар, расположенньй на уровне $j+1$ вправо от $b$, имеет партнера (или опору) на уровне $j$. Причем этот партнер расположен именно в тех клетках, куда мы собираемся передвигать наши яшики. То есть если шар $b$ был в клетке $(i, j+1)$, то партнеры интересующих нас шаров лежат в клетках $(\geqslant i, j)$. Отсюда все и видно.

Итак, случай с одной антицепю полностью разобран.

В.9. Общий случай. В принципе он доказьвается так же. Единственное, что нам 
нужно сделать, - это аккуратно разложить нашу $k$-антищепь в набор $k$ лент-антищепей. Это совсем отдельное рассуждение, не имеюшее никакого отношения к операциям $D$.

B.10. Pacnymblвание антицепей. Итак, пусть $A$ есть $k$-антищепь. Сновамы можем с каждым шаром включать в нашу $k$-антицеп все шары из его ящика. Тем самым мы снова будем понимать под $k$-антицепью набор яшиков $A$. Главное условие на $A$ состоит в том, что в нем нет цепей длины больше $k$.

Мы хотим показать, что найдутся $k$ лент-антицепей $A_{1}, \ldots, A_{k}$, которые не пересекаются и в совокупности покрьвают $A$. После этого мы можем заменить $A$ на $A_{1} \sqcup \cdots \sqcup A_{k}$, а сами ленты перенумеровать так, что $A_{1} \leqslant \cdots \leqslant A_{k}$ (т.е. $A_{1}$ - ближайшая к началу координат, $A_{2}$ - следуюшая, $\ldots, A_{k}$ - самая внешняя лента).

Делается это так. Идея состоит в том, чтобы в качестве $A_{k}$ (внешней ленты) взять "паретовскую гранищу" $A$. Более точно, определим $A_{k}^{\prime}$ как множество тех яшиков из $A$, северо-восточнее которых уже нет яшиков из $A$. Иначе говоря, это максимальнье ящики (относительно нашего строгого порядка). Это множество $A_{k}^{\prime}$ может не быть лентой, в нем могут быть пробелы. Тогда мы их заполним. А именно, если есть два ящика, мы вставляем крюк

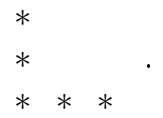

Обозначим получившуюся ленту как $A_{k}$. Почти очевидно, что множество $A \cup A_{k}$ снова является $k$-антицепью. Заменяя $A$ на это большее множество, мы смело можем считать, что $A_{k}$ является лентой.

Рассмотрим теперь $A^{\prime}=A \backslash A_{k}$. Мы утверждаем, что множество $A^{\prime}$ является $(k-1)$-антицепю. Это тоже фактически очевидно. Поэтому для него рассуждение можно повторить, отщепить ленту $A_{k-1}$, и т. д.

B.11. А теперь вернемся к ситуации с опусканием шара $b$ под действием $D$. Снова два случая. Либо этот шар при опускании снова попадает в яшик из $A$, и тогда все ясно. Либо нет. Скажем, шар $b$ лежал на ленте $A_{3}$. Тогда новая клетка, в которую он перемешается, не принадлежит предыдушей ленте $A_{2}$, как и все расположенные правее клетки. Если мы теперь заменим ленту $A_{3}$ на $A_{3}^{\prime}$, как в рассуждении с одной антицепью, эта лента $A_{3}^{\prime}$ снова будет проходить вьше $A_{2}$ (и, конечно, ниже $A_{4}$ ). И, очевидно, вес этой новой системы $A^{\prime}=A_{1} \sqcup A_{2} \sqcup A_{3}^{\prime} \sqcup A_{4} \sqcup \cdots$ будет не меншше, чем вес $A=A_{1} \sqcup A_{2} \sqcup A_{3} \sqcup A_{4} \sqcup \cdots$.

Итак, лемма В.7, а вместе с ней и теорема В.5 доказаны.

В.12. Приведем одно применение этой теоремы. Пусть $a$ - массив, и $* a$ - “перевернутый" массив (см. пп. 2.3 и 7.6). Тогда посет $\mathscr{P}(* a)$ - это посет, противоположншй $\mathscr{P}(a)$ (все отношения неравенства заменяются на противоположные). И хотя эти посеты в общем случае неизоморфны, антицепи у них одни и те же. Поэтому они имеют одну и ту же форму. Из теоремы В.5 следует, что массивы $a$ и $* a$ имеют одну и ту же форму. Это дает еше одно доказательство леммы 5.12.

\section{Дополнение С. Булевы массивы}

C.1. В этом дополнении мы кратко расскажем про теорию булевых массивов. Булев массив (формата $I \times J)$ - это массив $a=(a(i, j))$ чисел $a(i, j)$, равных 0 или 1 . Другими словами, булев массив - это подмножество в $I \times J$. Снова удобно понимать 
его как шары, разложенные по ящикам; однако теперь в каждом яшике может лежать только один шар. $I$-вес и $J$-вес массива определяются как раньше.

На множестве булевых массивов действуют операции $\widetilde{D}_{j}, \widetilde{U}_{j}, \widetilde{L}_{i}$ и $\widetilde{R}_{i}$, булевы варианты старых операций $D, U, L$ и $R$. Снова эти операции перемешают некоторьй шар вниз, вверх, влево или вправо. Однако они определяются иначе, чем раньше (хотя какую-то связь между определениями можно проследить). Дальнейшее развитие теории во многом идет параллельно предыдущей, поэтому мы в основном будем приводить лишш формулировки, опуская доказательства (подробности можно найти в [12]).

C.2. Определим операцию $\widetilde{D}_{j}$. Она, как и раньше, перемещает (если реально действует) некоторьй шар из строки $j+1$ в строчку $j$, перемешает вертикально вниз, сохраняя номер столбца, в котором этот шар лежал. Определение зависит только от этих двух строк, так что, как в п. 3.1, будем считать, что наш массив состоит только из двух строк: верхней $(a(\cdot, 2))$ и нижней $(a(\cdot, 1))$. Чтобы найти номер $i^{*}$ того столбца, из которого будет передвигаться (вниз) шар, мы воспользуемся терминологией рекордов. А именно, образуем функцию $\varphi=\varphi_{a}$ на множестве $I$ :

$$
\varphi(i)=a(1,2)-a(1,1)+\cdots+a(i, 2)-a(i, 1) .
$$

(Грубо говоря, это интеграл функции $a(\cdot, 2)-a(\cdot, 1)$.) Рекордом $i^{*}$ считается первая слева точка максимума функции $\varphi$. Если $\varphi\left(i^{*}\right) \leqslant 0$, операция $\widetilde{D}$ не действует, т.е. оставляет массив $a$ неизменным. Если же $\varphi\left(i^{*}\right)>0$, то мы берем шар в верхней ячейке $\left(i^{*}, 2\right)$ и перемешаем его в нижнюю ячейку $\left(i^{*}, 1\right)$. Заметим, что шар в ячейке $\left(i^{*}, 2\right)$ действительно имеется (так как $a\left(i^{*}, 2\right)-a\left(i^{*}, 1\right)>0$, что может быть, только если $a\left(i^{*}, 2\right)=1$ и $\left.a\left(i^{*}, 1\right)=0\right)$, а ячейка $\left(i^{*}, 1\right)$ пуста. Так что операция $\widetilde{D}$ переводит булев массив в булев.

Чтобы нагляднее представить все, лучше снова смотреть с точки зрения стабильного паросочетания, как в п. 3.4. Однако снова надо сделать небольшую, но необходимую модификацию. Когда верхний шар ищет себе нижнего партнера, то наиболее желательным для него является партнер, находящийся непосредственно под ним. Следующий по привлекательности левее, потом еще левее и т. д. Аналогично для нижних шаров самьй привлекательньй партнер - над ним, потом правее, правее и т. д. Схематично этот поиск партнеров можно изобразить как на рис. 14.

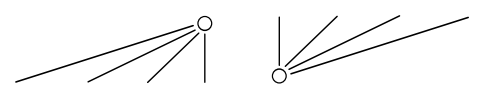

Рис. 14.

После того как образуется стабильное паросочетание, некоторые шары (вверху и внизу) могут остаться свободными, не нашедшими себе пару. При этом любой свободный верхний шар лежит строго левее любого свободного нижнего шара, как на рис. 15 .

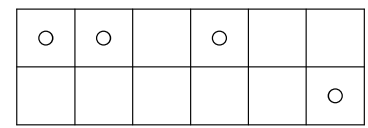

Рис. 15. 
Операция $\widetilde{D}$ опускает самьй правьй свободньй верхний шар. Операция $\widetilde{U}$, напротив, поднимает самьй левый свободный нижний шар. Видно, что операции $\widetilde{D}$ и $\widetilde{U}$ “почти обратны” друг к другу, в точности как старые $D$ и $U$.

С.3. Похожим образом определяются "горизонтальные" операции $\widetilde{L}$ и $\widetilde{R}$. И снова нужно поступить немного иначе, чем раньше. Нужно двухстолбцовый массив повернуть против часовой стрелки на 90 градусов, сделать $\widetilde{D}$ и потом вернуться назад (повернув уже по часовой стрелке на 90 градусов); так мы получим действие $\widetilde{L}$. Пример такой операции изображен на рис. 16.

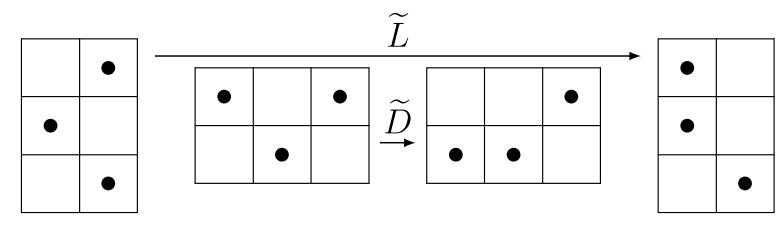

Рис. 16.

В терминах спаривания шары ишут партнеров в направлениях, приведенных на рис. 17 .

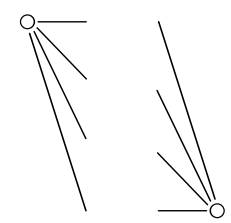

Рис. 17.

C.4. Главное следствие предыдущих причудливых определений - это то, что снова, как и в теореме 4.2 , вертикальные операции $\widetilde{L}_{i}$ и $\widetilde{R}_{i}$ коммутируют с горизонтальными $\widetilde{D}_{j}$ и $\widetilde{U}_{j}$. Доказывается это как и раньше. Мы не приводим доказательство, отсылая $\mathrm{K}[12]$.

Имея этот главньй факт, дальше все развивается формально как в части I для общих массивов. А именно, определим $\widetilde{\mathbf{D}}$-плотный булев массив как такой, на который все операции $\widetilde{D}_{j}$ действуют тривиально. Это означает, что для любой строчки $j+1$ любой шар в ней находит себе нижнего партнера. То есть что для любого $i$

$$
a(1, j+1)+\cdots+a(i, j+1) \leqslant a(1, j)+\cdots+a(i, j)
$$

В частности, строчные суммы для такого массива убывают с ростом номера строки, т.е. образуют разбиение (диаграмму Юнга). И если мы обычньм способом заполним клетки этой диаграммы символами из алфавита $I$, как в п. 2.6, мы увидим следующее. По горизонтали эти символы строго возрастают; это тривиальное следствие того, что в каждом яшике массива было не более одного шара. А по вертикали эти символы не убьвают; это просто переформулировка приведенного выше неравенства. То есть полученная “таблица" является антиполустандартной, точнее, она преврашается в полустандартную после транспонирования. 
Конечно, это рассуждение обратимо, и мы получаем следующий вариант предложения 9.3: существует каноническая биекция междумножеством С-плотных булевых массивов (формата $I \times J)$ и множеством полустандартных таблии, Юнга ( с п строками и алфавитом $J$ ).

C.5. Предыдущему результату стоит придать чуть иную форму. Будем представлять каждую строчку $a(\cdot, j)$ массива как подмножество в $I$. Пользуясь тем, что $I$ линейно упорядоченное, рассмотрим частичный порядок $\preceq$ на множестве $2^{I}$ подмножеств $I$. А именно, пусть даны два подмножества $S=\left\{i_{1}<\cdots<i_{s}\right\}$ и $T=\left\{i_{1}^{\prime}<\cdots<i_{t}^{\prime}\right\}$. Скажем, что $S \preceq T$, если $s \geqslant t$ и $i_{1} \leqslant i_{1}^{\prime}, \ldots, i_{t} \leqslant i_{t}^{\prime}$. Впрочем, это фактически отношение доминирования из п. 14.1. Так что задать $\widetilde{\mathbf{D}}$-плотный булев массив (формата $I \times J)$ - это то же самое, что задать возрастающую (в смысле доминирования $\preceq$ ) последовательность $S_{1} \preceq \cdots \preceq S_{m}$ подмножеств $I$.

Поясним примером. Пусть форма диаграммы равна $(4,3,1)$ и она заполнена как

2

236

$1 \quad 3 \quad 4 \quad 5$

Тогда соответствующий $\widetilde{\mathbf{D}}$-плотный массив $a$ имеет вид

$$
\begin{array}{lllllll}
0 & 1 & 0 & 0 & 0 & 0 & \\
0 & 1 & 1 & 0 & 0 & 1 \\
1 & 0 & 1 & 1 & 1 & 0
\end{array} .
$$

"Антитаблица" просто указьвает - куда, на какую позищию (по горизонтали) должен уехать каждый шар в строке. Мы видим также, что наш массив имеет "слоистую" структуру. Она образована образами вертикальных колонок диаграммы Юнга. Или, если посмотреть наоборот, - диаграмма получена "уплотнением влево" нашего булева массива $а$. И это свойственно не только данному примеру, это обший факт.

C.6. Аналогично можно поступить и с $\widetilde{\mathbf{L}}$-плотньми массивами. Снова такие массивы соответствуют полустандартньм (на этот раз настоящим) таблищам Юнга. Однако нужно помнить о тех кувырканиях, которые следует произвести. В результате рецепт построения таблицы по массиву выглядит так. Таблицу лучше всего рисовать в английской традиции. Столбцы массива соответствуют столбцам диаграммы (а то, что получается действительно диаграмма, следует из того простого факта, что $I$-вес $\widetilde{\mathbf{L}}$-плотного массива есть убьвающая последовательность). Надо двигаться по столбцу массива сверху вниз и, встретив шар, вписьвать в столбец диаграммы номер строки, считая его сверху.

Снова поясним примером. Пусть массив $а$ имеет вид

$$
\begin{array}{llll}
1 & 1 & 0 & 0 \\
0 & 0 & 1 & 0 \\
1 & 0 & 0 & 1 \\
0 & 1 & 1 & 1 \\
1 & 0 & 0 & 0
\end{array} .
$$

Легко проверить, что он действительно $\widetilde{\mathbf{L}}$-плотньй (и увидеть в нем “горизонтально-слоистую” структуру). Двигаясь сверху вниз по первому столбцу, мы получаем 
первьй столбец таблицы $(135)^{T}$. Окончательная таблица (в английской записи) имеет вид

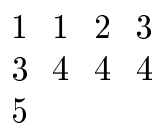

или, в наших обычных обозначениях,

5

$\begin{array}{llllll}3 & 4 & 4 & 4\end{array}$

$1 \quad 1 \quad 2 \quad 3$

С.7. Наконец, нужно сказать, что биплотньй булев массив выглядит просто как набор шаров, расположенных в некоторой диаграмме Юнга. В самом деле, пусть $a-$ булев массив, которьй одновременно $\widetilde{\mathbf{D}}$-плотньй и $\widetilde{\mathbf{L}}$-плотньй. Рассмотрим шар в первом столбце. В силу $\widetilde{\mathbf{D}}$-плотности у него должна быть опора на этаже ниже, расположенная (нестрого) левее. Но так как он находится в первом столбце, то эта опора может быть только под ним. Это значит, что шары в первом столбце идут подряд.

Аналогично с шарами во втором столбце. Возьмем такой шар (если он есть). Заметим, что он не может располагаться вьше всех шаров из первого столбца, поскольку у него должна быть опора слева и вьше в силу $\widetilde{\mathbf{L}}$-плотности. У него также должна быть опора этажом ниже, расположенная под ним или левее. Шар из первого столбца не может служить ему опорой, так как у него есть лучший партнер, расположенньй над ним (см. выше). Поэтому прямо под ним должен располагаться шар из массива.

Аналогично с третьим столбцом и так далее. Мы видим, что для каждого шара в ячейке $(i, j)$ имеется шар в ячейке $(i, j-1)$ (если $j>1$ ) и шар в ячейке $\left(i-1, j^{\prime}\right)$ с $j^{\prime} \geqslant j$ (если $i>1)$. Отсюда, конечно, следует, что если заполнена ячейка $(i, j)$, то заполнены и все меньшие (относительно порядка на $I \times J)$ ячейки. То есть что носитель нашего массива - диаграмма Юнга.

С.8. Как и в разделе 5 , действуя операциями $\widetilde{D}_{j}$, мы любой булев массив можем уплотнить и получить $\widetilde{\mathbf{D}}$-плотный массив $\widetilde{\mathbf{D}}(a)$. Аналогично получается $\widetilde{\mathbf{L}}$-плотнњй массив $\widetilde{\mathbf{L}}(a)$. Конечно, массивы $\widetilde{\mathbf{D}}(\widetilde{\mathbf{L}}(a))$ и $\widetilde{\mathbf{L}}(\widetilde{\mathbf{D}}(a))$ совпадают (а носитель этого массива называется формой исходного массива $a$ ). Как в разделе 6 , мы имеем биекцию. Иначе говоря, задать булев массив $a$ - то же самое, что задать диаграмму Юнга (форму $\lambda$ ) и два его заполнения - полустандартное (соответствуюшее $\widetilde{\mathbf{L}}(a)$ ) и антиполустандартное (соответствуюшее $\widetilde{\mathbf{D}}(a)$ ). Или, другими словами, задать полустандартную таблицу формы $\lambda$ и полустандартную таблицу сопряженной формы $\lambda^{\prime}$.

Эта биекция совпадает с так назьваемьм дуальным соответствием Кнута.

Еще одно замечание. Ясно, что вертикальные операции $\widetilde{D}_{j}$ сохраняют $I$-вес массива. С другой стороны, рассматривая $\widetilde{\mathbf{D}}$-плотньй массив (см. п. С.5), мы видим, что его вес более "равномерно распределен”, чем вес его формы, т.е. чем сопряженное разбиение $\lambda^{\prime}$. На формальном языке, вес мажсорируется вектором $\lambda^{\prime}$. Так что $\mu=w t_{I}(a) \preceq \lambda^{\prime}$. Симметрично мы имеем для $J$-веса $\nu=w t_{J}(a) \preceq \lambda$. Вместе это дает, что $\nu \preceq \lambda \preceq \mu^{\prime}$. Так что если $a-$ булев массив, мыполучаем, что вектор $\nu$ строчных сумм мажорируется вектором $\mu^{\prime}$, сопряженным к вектору столбцовых сумм $\mu$. Легко показать, что верно и обратное (см. п. 12.1), и мы получаем теорему, установленную Д. Гейлом и Г. Райзером (см. подробнее [1], [42]): булева матрица с предписаннымми строчньлм суммами и и столбцовыми суммами н существует тогда и только 
тогда, когда $\nu \preceq \mu^{\prime}$. Приведенная вьше биекция дает более точньй ответ о числе таких матриц (см. [40; $§ 6$, гл. 1]).

Рассуждая как в п. 13.6, мыполучаем “дуальную” к формуле Коши форлулу Литтлвуда:

$$
\prod_{1 \leqslant i \leqslant n, 1 \leqslant j \leqslant m}\left(1+x_{i} y_{j}\right)=\sum_{\lambda} s_{\lambda^{\prime}}\left(x_{1}, \ldots, x_{n}\right) s_{\lambda}\left(y_{1}, \ldots, y_{m}\right)
$$

C.9. Наконец, на булевы массивы и соответствуюшие операции можно посмотреть с точки зрения теоремы Грина (см. дополнение В). Свяжем с булевым массивом $a$ посет $\widetilde{\mathbf{P}}(a)$. Как множество он устроен как и раньше и состоит их всех шаров нашего массива (или равен носителю массива $a$ ). Однако порядок на нем задается несколько иначе. А именно, мы считаем, что шар в ячейке $(i, j)$ больше шара в ячейке $\left(i^{\prime}, j^{\prime}\right)$, если $i^{\prime} \leqslant i$ и $j^{\prime}<j$.

Имеет место факт, аналогичньй теореме В.5. А именно, что форма посета $\widetilde{\mathbf{P}}(a)$ совпадает с формой булева массива $\lambda$, определенной в предыдушем пункте. Схема доказательства та же. Нужно только сказать, что антицепи представляются теперь не как ленты из п. В.8, а как “горизонтальные полосы”. Довольно ясно, как нужно менять антищепь, если мы производим опускание некоторого шара под действием операции $\widetilde{D}_{j}$. Нужно взять брусок $B$ как в п. В.8, но перемешать его не на вектор $(-1,-1)$, как раньше, а на вектор $(0,-1)$, т.е. вертикально вниз.

\section{СПИСОК ЛИТЕРАТУРЫ}

[1] М. Айгнер. Комбинаторная теория. М.: Мир, 1982.

[2] S. Ariki. Robinson-Schensted correspondence and left cells // Adv. Stud. Pure Math. 2000. V. 28. P. 1-20; arXiv: math.QA/9910117.

[3] E.A. Bender, D.E. Knuth. Enumeration of plane partitions // J. Combin. Theory Ser. A. 1972. V. 13. P. 40-54.

[4] G. Benkart, F. Sottile, J. Stroomer. Tableau switching: algorithms and applications // J. Combin. Theory Ser. A. 1996. V. 76. №1. P. 11-43.

[5] A. D. Berenstein, A. V. Zelevinsky. Triple multiplicities for $\mathrm{sl}(r+1)$ and the spectrum of the exterior algebra of the adjoint representation // J. Algebraic Combin. 1992. V. 1. № 1. P. 7-22.

[6] A. Berenstein, A. Zelevinsky. Tensor product multiplicities, canonical bases and totally positive varieties // Invent. Math. 2001. V. 143. №1. P. 77-128.

[7] T. Britz, S. Fomin. Finite posets and Ferrers shapes // Adv. Math. 2001. V. 158. №1. P. 86-127.

[8] A.S. Buch (with an appendix by W. Fulton). The saturation conjecture (after A. Knutson and T. Tao) // Enseign. Math. (2). 2000. V. 46. №1-2. P. 43-60.

[9] V. Danilov, A. Karzanov, G. Koshevoy. Discrete strip-concave functions, GefandTsetlin patterns, and related polyhedra // arXiv: math.CO/0404298.

[10] В.И. Данилов, Г.А. Кошевой. Нилпотентные операторы и дискретно вогнутые функции // Изв. РАН. Сер. матем. 2003. Т. 67. №1. С. 3-20.

[11] В. И. Данилов, Г. А. Кошевой. Дискретная выпуклость и эрмитовы матрицы // Труды МИАН. 2003. Т. 241. С. 68-89.

[12] V. Danilov, G. Koshevoy. Bicrystals and crystal $G L(V), G L(W)$-duality // Preprint RIMS-1485. Kyoto: Kyoto University, 2004.

[13] V. Danilov, G. Koshevoy. Discrete convexity and unimodularity. I // Adv. Math. 2004. V. 189. № 2. P. 301-324.

[14] V. Danilov, G. Koshevoy. A simple proof of associativity and commutativity of LR-coefficients (or the hive ring) // arXiv: math.CO/0409447. 
[15] M. Date, M. Jimbo, T. Miwa. Representations of $U_{q}(\mathfrak{g l}(n, C))$ at $q=0$ and the Robinson-Schensted correspondence // Physics and Mathematics of Strings / ed. L. Brink, D. Friedman, A. M. Polyakov. Teaneck, NJ: Word Scientific, 1990. P. 185-211.

[16] J. A. De Loera, T. B. McAllister. Vertices of Gelfand-Tsetlin polytopes // Discrete Comput. Geom. 2004. V. 32. № 4. P. 459-470; arXiv: math.CO/0309329.

[17] P. Deift. Integrable systems and combitatorial theory // Notices Amer. Math. Soc. 2000. V. 47. №6. P. 631-640.

[18] С. В. Фомин. Конечные частично упорядоченные множества и диаграммы Юнга // Докл. AH CCCP. 1978. T. 243. № 5. C. 1144-1147.

[19] S. Fomin. Knuth equivalence, Jeu de taquin, and the Littlewood-Richardson rule. Appendix 1 // R. P. Stanley. Enumerative Combinatorics. V. 2. Cambridge: Cambridge Univ. Press, 1999.

[20] S. Fomin, A. Zelevinsky. The Laurent phenomenon // Adv. in Appl. Math. 2002. V. 28. №2. P. 119-144.

[21] W. Fulton. Young Tableaux. Cambridge: Cambridge Univ. Press, 1997. (London Math. Soc. Stud. Texts. V. 35.)

[22] W. Fulton. Eigenvalues, invariant factors, highest weights, and Schubert calculus // Bull. Amer. Math. Soc. (N.S.). 2000. V. 37. №3. P. 209-249.

[23] W. Fulton, J. Harris. Representation Theory. A first course. New York: Springer-Verlag, 1991.

[24] O. Gleizer, A. Postnikov. Littlewood-Richardson coefficients via Yang-Baxter equation // Internat. Math. Res. Notices. 2000. V. 14. P. 741-774; arXiv: math.QA/9909124.

[25] C. Greene. An extension of Schensted's theorem // Adv. Math. 1974. V. 14. P. 254-265.

[26] Ф. Грифффитс, Дж. Харрис. Принципы алгебраической геометрии. Т. 1, 2. М.: Мир, 1982.

[27] M. Haiman. Dual equivalence with applications, including a conjecture of Proctor // Discrete Math. 1992. V. 99. № 1-3. P. 79-113.

[28] A. Henriques, J. Kamnitzer. The octahedron recurrence and $g l(n)$ cristals // arXiv: math.CO/0408114.

[29] Г. Джеймс. Теория представлений симметрических групп. М.: Мир, 1982.

[30] M. Kashiwara. On crystal bases // CMS Conf. Proc. 1995. V. 16. P. 155-197.

[31] M. Kashiwara. Bases Cristallines des Groupes Quantiques. Paris: Société Math. France, 2002. (Cours Spéc. V. 9.)

[32] A. N. Kirillov. Introduction to tropical combinatorics // Physics and Combinatorics. Proceedings of the Nagoya 2000 2nd International Workshop / ed. A. N. Kirillov, N. Liskova. Singapore: World Scientific, 2001. P. 82-150.

[33] A. N. Kirillov, A.D. Berenstein. Groups generated by involutions, Gelfand-Tsetlin patterns, and combinatorics of Young tableaux // Алгебра и анализ. 1995. Т. 7. №1. C. $92-152$.

[34] D. E. Knuth. Permutations, matrices, and generalized Young tableaux // Pacific J. Math. 1970. V. 34. P. 709-727.

[35] A. Knutson, T. Tao. The honeycomb model of $\mathrm{GL}_{n}(\mathbb{C})$ tensor products. I: Proof of the saturation conjecture // J. Amer. Math. Soc. 1999. V. 12. №4. P. 1055-1090.

[36] A. Knutson, T. Tao, C. Woodward. A positive proof of the Littlewood-Richardson rule using the octahedron recurrence // Electron. J. Combin. 2004. V. 11. Research Paper 61; arXive: math.CO/0306274.

[37] A. Lascoux. Double crystal graphs // Studies in Memory of Issai Schur / ed. A. Joseph et al. Boston: Birkhäuser, 2003. P. 95-114. (Progr. Math. V. 210.)

[38] A. Lascoux, B. Leclerc, J.-Y. Thibon. The plactic monoid // Algebraic Combinatorics on Words. Cambridge: Cambridge Univ. Press, 2002. P. 164-196. (Encyclopedia Math. Appl. V. 90.)

[39] A. Lascoux, M. P. Schützenberger. Le monoïde plaxique // Non-commutative Structures in Algebra and Geometric Combinatorics (Naples, 1978). Rome: CNR, 1981. P. 129-156. (Quad. Ric. Sci. V. 109.)

[40] И. Макдональд. Симметрические функции и многочлены Холла. М.: Мир, 1985.

[41] Ю. И. Манин. Доказуемое и недоказуемое. М.: Сов. радио, 1979. 
[42] А. Маршалл, И. Олкин. Неравенства: теория мажоризации и ее приложения. М.: Мир, 1983.

[43] А. Ю. Окуньков, А. М. Вершик. Новьй подход к теории представлений симметрических групп. 2 // Записки научн. семинаров ПОМИ. 2004. Т. 307. С. 57-98.

[44] I. Pak, E. Vallejo. Reductions of Young tableau bijections // arXiv: math.CO/0408171.

[45] B. E. Sagan. The Symmetric Group. Pacific Grove, CA: Wadsworth \& Brooks/Cole, 1991.

[46] C. Schensted. Longest increasing and descreasing subsequences // Canad. J. Math. 1961. V. 13. P. $179-191$.

[47] M.P. Schützenberger. Promotion des morphismes d'ensembles ordonnés // Discrete Math. 1972. V. 2. P. 73-94.

[48] R.P. Stanley. Enumerative Combinatorics. V. 2. Cambridge: Cambridge Univ. Press, 1999. (With a foreword by G.-C. Rota and Appendix 1 by S. Fomin.)

[49] R. Steinberg. An occurrence of the Robinson-Schensted correspondence // J. Algebra. 1988. V. 113. №2. P. 523-528.

[50] J. R. Stembridge. Rational tableaux and the tensor algebra of $\mathrm{gl}_{n} / / \mathrm{J}$. Combin. Theory Ser. A. 1987. V. 46. №1. P. 79-120.

[51] M. A. A. van Leeuwen. The Littlewood-Richardson rule, and related combinatorics // MSJ Mem. 2001. V. 11. P. 95-145; arXiv: math. CO/9908099.

[52] M. A. A. van Leeuwen. The Robinson-Schensted and Schützenberger algorithms, an elementary approach // Electronic J. Combin. 1996. V. 3. №2. Research Paper 15.

[53] А. В. Зелевинский. Обобщенные коэффициенты Литтлвуда-Ричардсона, канонические базисы и полная положительность // Глобус. Общематематический семинар. Вып. 1 / ред. В. В. Прасолов, М.А. Цфасман. М.: МЦНМО, 2004. С. 147-160.

Центральньй экономико-математический институт РАН

Поступила в редакцию

E-mail: vdanilov43@mail.ru; koshevoy@cemi.rssi.ru

14.07.2004 